\title{
Wild Bootstrap Seasonal Unit Root Tests for Time Series with Periodic Non-Stationary Volatility*
}

\author{
Giuseppe Cavaliere ${ }^{a}$, Anton Skrobotov ${ }^{b}$ and A.M. Robert Taylor ${ }^{c}$ \\ ${ }^{a}$ Department of Statistical Sciences, University of Bologna \\ ${ }^{b}$ Russian Presidential Academy of National Economy and Public Administration \\ ${ }^{c}$ Essex Business School, University of Essex
}

February 21, 2017

\begin{abstract}
We investigate the behaviour of the well-known HEGY (Hylleberg, Engle, Granger and Yoo, 1990, Journal of Econometrics, vol.44, pp.215-238) regression-based seasonal unit root tests in cases where the driving shocks can display periodic non-stationary volatility and conditional heteroskedasticity. Our set up allows for periodic heteroskedasticity, non-stationary volatility and (seasonal) GARCH as special cases. We show that the limiting null distributions of the HEGY tests depend, in general, on nuisance parameters which derive from the underlying volatility process. Monte Carlo simulations show that the standard HEGY tests can be substantially over-sized in the presence of such effects. As a consequence, we propose wild bootstrap implementations of the HEGY tests. Two possible wild bootstrap re-sampling schemes are discussed, both of which are shown to deliver asymptotically pivotal inference under our general conditions on the shocks. Simulation evidence is presented which suggests that our proposed bootstrap tests perform well in practice, largely correcting the size problems seen with the standard HEGY tests even under extreme patterns of heteroskedasticity, yet not losing finite sample relative to the standard HEGY tests.
\end{abstract}

Keywords: seasonal unit roots, (periodic) non-stationary volatility, conditional heteroskedasticity, wild bootstrap.

JEL Codes: C12, C22.

\section{Introduction}

Over the last three decades, a debate has been conducted in the literature as to whether the withinyear variations in seasonally observed time series processes are deterministic or attributable to unit

\footnotetext{
${ }^{*}$ We thank the Editor, Essie Massoumi, an Associate Editor and two anonymous referees for their helpful and constructive comments on earlier versions of this paper. Cavaliere and Taylor thank the Danish Council for Independent Research, Sapere Aude | DFF Advanced Grant (Grant nr: 12-124980) for financial support. Correspondence to: Robert Taylor, Essex Business School, University of Essex, Wivenhoe Park, Colchester, CO4 3SQ, UK. Email: rtaylor@essex.ac.uk
} 
roots at the seasonal frequency components of the data. This question is important because incorrect modelling of the seasonality has serious implications for the statistical validity of any subsequent procedures. Moreover, most available seasonally adjusted data are based on filtering methods which imply the application of seasonal differencing to the data. If the data do not contain seasonal unit roots then the resulting seasonally adjusted data will contain moving average unit roots, rendering standard autoregressive modelling methods invalid. In order to formally investigate this issue, in the seminal paper in this literature, Hylleberg, Engle, Granger and Yoo (1990) [HEGY] propose a seasonal generalisation of the augmented Dickey-Fuller [ADF] unit root test under the assumption of homoskedastic innovations. This procedure allows the practitioner to test for unit root behaviour at each of the zero and seasonal frequency components of the data, either separately or via a joint test.

A large body of recent applied work has grown suggesting that the assumption of constant unconditional volatility is at odds with what is observed in time series data for many macroeconomic and financial series. In particular, a general decline in the unconditional volatility of the shocks driving macroeconomic series in the twenty years or so leading up to the recent financial crisis has been a relatively commonly observed phenomenon. This feature is known as the "great moderation"; see, inter alia, Kim and Nelson (1999), McConnell and Perez-Quiros (2000), Sensier and van Dijk (2004), and references therein. In the non-seasonal case it is well documented that permanent changes in volatility (so that the volatility process becomes non-stationary) of this form can considerably impact upon unit root and co-integration tests. In particular, Cavaliere (2004), Cavaliere and Taylor (2007, 2008a,b) and Beare (2008), among others, show that the limiting distributions of widely used unit root test statistics, such as ADF statistics, depend on a particular function, the so-called variance profile, of the underlying volatility process which leads to tests which are incorrectly sized, even in the limit. A number of possible solutions to this inference problem have been proposed. Arguably most successfully, Cavaliere and Taylor (2008a) propose wild bootstrap implementations of standard unit root tests which they show to correctly replicate the limiting null distribution of the original statistics, thereby yielding asymptotically pivotal unit root inference under non-stationary volatility.

Since the seminal study of HEGY, seasonal unit root testing has received a large amount of attention in the literature. Recent contributions include del Barrio Castro et al. (2012, 2016), Rodrigues and Taylor (2007), Rodrigues and Taylor (2004) and Smith et al. (2009). However, few studies have considered the influence of heteroskedasticity on the HEGY tests. A leading exception is Burridge and Taylor (2001a) who focus attention on the impact of periodic (or seasonal) heteroskedasticity [hereafter, $\mathrm{PH}]$ in the innovations on the HEGY tests. PH occurs where the unconditional variance of the innovations in any given season is constant across years, but varies across seasons. Under the seasonal unit root null hypothesis, Burridge and Taylor (2001a) demonstrate that the limiting distributions of the tests for zero and Nyquist frequency unit roots are unaffected by PH. However, they show this is not the case for tests for unit roots at the harmonic seasonal frequencies, or any joint frequency tests which involve the harmonic frequency. In response to this problem, Burridge and Taylor (2004) propose an i.i.d. bootstrap implementation of the HEGY seasonal unit root tests, based on i.i.d. resampling (with replacement) separately for each season from the residuals from the estimated HEGY 
regression. While this approach delivers asymptotically valid tests under $\mathrm{PH}$, it will not be valid under the more non-stationary volatility framework discussed in the context of non-seasonal unit root testing above. More recently, Zou and Politis (2016) have proposed further i.i.d. bootstrap implementations of augmented HEGY tests as well as block bootstrap implementations of unaugmented HEGY tests and shown their validity under PH (also allowing for conditional heteroskedasticity). They do not, however, allow for periodic non-stationary volatility as considered here.

Our aim in this paper is to generalise the approach of Cavaliere and Taylor (2008a) to the seasonal context. In particular, we will adopt a periodic non-stationary volatility set-up which includes both the form of PH considered in Burridge and Taylor (2001a) and the non-stationary volatility set-up of, inter alia, Cavaliere and Taylor (2008a) as special cases. Indeed, under our set-up both can occur, such that the relative pattern of PH between the seasons could change across the sample. Our set-up also allows for conditional heteroskedasticity in the innovations. Under our periodic non-stationary volatility set-up we first derive the large sample behaviour of the HEGY seasonal unit root tests demonstrating that these are not, in general, pivotal depending on nuisance parameters arising from the heteroskedasticity present in the innovations. We show that asymptotically pivotal inference under the periodic non-stationary volatility set-up we consider can however be attained using wild bootstrap based implementations of the HEGY tests. We suggest two possible, asymptotically equivalent, wild bootstrap re-sampling schemes, the first a conventional wild bootstrap device whereby each residual from the fitted HEGY regression is multiplied by a different external random variable, and the second a seasonal block wild device whereby within a given year each residual from the fitted HEGY regression is multiplied by the same wild bootstrap shock. Monte Carlo simulations for a variety of (periodic) non-constant volatility models suggest that the wild bootstrap HEGY tests perform very well in practice with only small finite sample differences between the two wild bootstrap schemes. We also outline how the re-scaled information-based lag length selection methods of Cavaliere et al. (2015) can be adapted to the seasonal unit root testing case.

The outline of the remainder of the paper is as follows. In section 2 the heteroskedastic seasonal model and the underlying assumptions we make concerning it are outlined. In section 3 the seasonal unit root hypotheses of concern and the associated HEGY tests are detailed. Here we also detail the limiting null distributions of the HEGY tests under the periodic non-constant volatility formulation we adopt in this paper. The wild bootstrap algorithm (based on either of the two re-sampling devices discussed above) and the limiting distributions of the associated wild bootstrap HEGY statistics are detailed in section 4. Section 5 presents the results of our Monte Carlo study into the relative finite sample size and power properties of the HEGY tests and our proposed wild bootstrap HEGY tests. Results are presented in the main text for the conventional wild bootstrap re-sampling device, with the corresponding results for the seasonal block wild re-sampling device reported in an on-line supplementary appendix. Lag length selection in the context of the HEGY regression is also discussed in section 5. Section 6 concludes. Mathematical proofs are reported in the supplementary appendix. 


\section{The Heteroskedastic Seasonal Model}

Consider the univariate seasonal time series $\left\{x_{S n+s}\right\}$, which satisfies the data generating process [DGP]

$$
\begin{aligned}
\alpha(L) x_{S n+s} & =u_{S n+s}, s=1-S, \ldots, 0, n=2, \ldots, N \\
\phi(L) u_{S n+s} & =\varepsilon_{S n+s} \\
\varepsilon_{S n+s} & =\sigma_{S n+s} e_{S n+s}
\end{aligned}
$$

where $S$ denotes the number of seasons, $\alpha(L)=1-\sum_{j=1}^{S} \alpha_{j} L^{j}$ is an $S$-order autoregressive polynomial, $\phi(L)=1-\sum_{j=1}^{p} \phi_{j} L^{j}$ is a $p$ th order autoregressive polynomial, $L$ is the lag operator such that $L^{S j+k} y_{S n+s}=y_{S(n-j)+s-k}$. The total sample size is $T:=S N, N$ representing the total number of seasonal cycles (eg years) completed. For simplicity we will refer to a complete seasonal cycle as a year in what follows. For the present we follow Rodrigues and Taylor (2007) and assume that the initial conditions, $x_{1}, \ldots, x_{S}$, are of $o_{p}\left(N^{1 / 2}\right)$; relaxation of this condition is discussed in Remark 11, below.

Consider the vector-of-seasons innovation process $\left\{\mathcal{E}_{n}\right\}$ where $\mathcal{E}_{n}:=\left(\varepsilon_{S n-(S-1)}, \varepsilon_{S n-(S-2)}, \ldots, \varepsilon_{S n}\right)^{\prime}$, $n=1, \ldots, N$. We then assume that $\left\{\mathcal{E}_{n}\right\}$ satisfies the following assumption, which is a special case of Assumption 2 of Boswijk et al. (2016).

Assumption 1 The innovation process $\left\{\mathcal{E}_{n}\right\}$ satisfies $\mathcal{E}_{n}:=\boldsymbol{\Omega}_{n} \mathbf{E}_{n}$, where $\mathbf{E}_{n}:=\operatorname{diag}\left\{e_{S n-(S-1)}, \ldots, e_{S n}\right\}$ and $\boldsymbol{\Omega}_{n}:=\operatorname{diag}\left\{\sigma_{S n-(S-1)}, \ldots, \sigma_{S n}\right\}$ is an $S \times S$ non-stochastic matrix which satisfies $\boldsymbol{\Omega}_{n}:=\boldsymbol{\Omega}(n / N):=$ $\operatorname{diag}\left\{\sigma_{1-S}(n / N), \ldots, \sigma_{0}(n / N)\right\}$ for all $n=1, \ldots, N$, where $\boldsymbol{\Omega}(\cdot) \in \mathcal{D}_{\mathbb{R}^{S \times S}}[0,1]$, where $\mathcal{D}_{\mathbb{R}^{m \times n}}[0,1]$ is used to denote the space of $m \times n$ real matrices of càdlàg functions on $[0,1]$, and $\mathbf{\Upsilon}(u):=\boldsymbol{\Omega}(u) \boldsymbol{\Omega}(u)^{\prime}$ is assumed to be positive definite for all $u \in[0,1]$; the innovations $\left\{e_{S n+s}\right\}$ form a martingale difference sequence $[M D S]$ with respect to the filtration $\mathcal{F}_{S n+s}=\sigma\left(\varepsilon_{1}, \ldots, \varepsilon_{S n+s}\right)$ with conditional variance $h_{S n+s}:=$ $E\left(e_{S n+s}^{2} \mid \mathcal{F}_{S n+s-1}\right)$, satisfying $\sup _{n, s} E\left(e_{S n+s}^{4 r}\right)<\infty$ for some $r>1$, and $N^{-1} \sum_{n=1}^{N} h_{S n+s} \stackrel{p}{\rightarrow} E\left(h_{S n+s}\right)=$ $1, s=1-S, \ldots, 0$, where $\stackrel{p}{\rightarrow}$ denotes convergence in probability as $N \rightarrow \infty$.

Remark 1 A consequence of Assumption 1 is that $\mathcal{E}_{n}$ is an $S$-dimensional vector MDS with respect to $\mathcal{F}_{n}^{S}:=\mathcal{F}_{S n}$, with conditional variance matrix $\Upsilon_{n \mid n-1}:=E\left(\mathcal{E}_{n} \mathcal{E}_{n}^{\prime} \mid \mathcal{F}_{n-1}^{S}\right)=\boldsymbol{\Omega}_{n} \mathbf{h}_{n} \boldsymbol{\Omega}_{n}^{\prime}$, where $\mathbf{h}_{n}:=$ $E\left(\mathbf{E}_{n} \mathbf{E}_{n}^{\prime} \mid \mathcal{F}_{n-1}^{S}\right)$, and time-varying unconditional variance matrix $\boldsymbol{\Upsilon}_{n}:=E\left(\mathcal{E}_{n} \mathcal{E}_{n}^{\prime}\right)=\boldsymbol{\Omega}_{n} \boldsymbol{\Omega}_{n}^{\prime}>0 .^{1}$ As such, Assumption 1 combines both stationary conditional heteroskedasticity and non-stationary unconditional volatility. These are obtained in isolation of each other as special cases with $\boldsymbol{\Omega}(u)=\boldsymbol{\Omega}$ (constant unconditional variance, and hence only conditional heteroskedasticity) and $\mathbf{h}_{n}=\mathbf{I}_{S}$ (so that $\boldsymbol{\Upsilon}_{n \mid n-1}=\boldsymbol{\Upsilon}_{n}=\mathbf{\Upsilon}(n / N)$, allowing only unconditional non-stationary volatility). As discussed in Cavaliere et al. (2010), the latter implies that the elements of $\boldsymbol{\Upsilon}_{n}$ are only required to be nonstochastic, bounded and to display a countable number of jumps, therefore allowing for an extremely

\footnotetext{
${ }^{1}$ The condition imposed by Assumption 1 that $E\left(h_{S n+s}\right)=1$, implies that $E\left(\mathbf{E}_{n} \mathbf{E}_{n}^{\prime}\right)=I_{S}$, where $I_{k}$ denotes the $k \times k$ identity matrix. This restriction entails no loss of generality, however, because the leading diagonal elements of $\boldsymbol{\Omega}_{n}$ are unrestricted, and is made only to simplify notation. In particular, any $\mathcal{E}_{n}=\boldsymbol{\Omega}_{n} \mathbf{E}_{n}$ satisfying Assumption 1 with $E\left(\mathbf{E}_{n} \mathbf{E}_{n}^{\prime}\right)=\boldsymbol{\Xi}$, where $\boldsymbol{\Xi}$ is diagonal, can also be expressed as $\mathcal{E}_{n}=\tilde{\boldsymbol{\Omega}}_{n} \tilde{\mathbf{E}}_{n}$ with $E\left(\tilde{\mathbf{E}}_{n} \tilde{\mathbf{E}}_{n}^{\prime}\right)=I_{S}$ and $\tilde{\boldsymbol{\Omega}}_{n}:=\boldsymbol{\Omega}_{n} \boldsymbol{\Xi}^{-1 / 2}$, where both $\tilde{\mathbf{E}}_{n}$ and $\tilde{\boldsymbol{\Omega}}_{n}$ satisfy Assumption 1 .
} 
wide class of potential models for the behaviour of the variance matrix of $\mathcal{E}_{n}$ including single or multiple variance shifts, variances which follow a broken trend, and smooth transition variance shifts. The former allows for a wide range of conditionally heteroskedastic processes including stochastic volatility and generalised autoregressive-conditional heteroskedasticity (GARCH) processes; see Boswijk et al. (2016) for further discussion.

Remark 2 Assumption 1 allows for the case where time-varying behaviour occurs in either some or all of the seasons; for example the innovation variance in the first season might display a single break at some point in the sample, but the innovation variance in the remaining seasons need not display a break. The pattern of $\mathrm{PH}$ across seasons is also permitted to change through the sample under Assumption 1, allowing for example the case where $\mathrm{PH}$ is absent in some parts of the sample but present in others. Notice the case where $\boldsymbol{\Omega}_{n}:=\operatorname{diag}\left\{\sigma_{(1-S)}, \ldots, \sigma_{0}\right\}$ and $\mathbf{h}_{n}=I_{S}$, corresponds to the particular PH case considered in Burridge and Taylor (2001a), while $\boldsymbol{\Omega}_{n}:=\sigma I_{S}$ and $\mathbf{h}_{n}=I_{S}$, $n=1, \ldots, N$, corresponds to the (conditionally and, hence, unconditionally) homoskedastic case.

To complete the assumptions needed on $u_{t}$, we place the following conditions on $\phi(L)$ in $(2)$.

Assumption 2 The pth order lag polynomial $\phi(z)$ satisfies: (a) $0 \leq p<\infty$, and (b) $\phi(z) \neq 0$ for all $|z| \leq 1$.

Remark 3 Assumption 2, which imposes that $\phi(z)$ is a stationary finite-order polynomial, is standard in this literature and coincides with Assumption 3.1(c) of Burridge and Taylor (2001a). Assumption 2 guarantees that $\phi(z)$ has the unique inverse $\psi(z):=1+\sum_{j=1}^{\infty} \psi_{j} z^{j}$.

Remark 4 Our focus in this paper is on developing wild bootstrap implementations of parametric lag-augmented HEGY tests which are asymptotically valid under Assumption 1. Accordingly, weak dependence in $\left\{u_{S n+s}\right\}$ is specified parametrically. An alternative approach is considered in Zou and Politis (2016) for the case where $\left\{\mathcal{E}_{n}\right\}$ is instead formulated as a strong mixing process. For this case, Zou and Politis (2016) develop seasonal block bootstrap implementations of the HEGY tests which obtain from an un-augmented HEGY regression, corresponding to equation (9) below but with the lagged dependent variables, $\left\{\Delta_{S} x_{S n+s-j}\right\}_{j=1}^{p}$, omitted from the set of regressors. Under the usual condition that the block length increases with the sample size, they demonstrate the asymptotic validity of these block bootstrap tests under conditions which crucially do not allow for non-stationary volatility; that is, $\boldsymbol{\Omega}_{n}$ must be time-invariant for the asymptotic validity of the block bootstrap HEGY tests of Zou and Politis (2016) to hold.

\section{Seasonal Unit Root Testing}

\subsection{Seasonal Unit Root Hypotheses}

Our aim in this paper is to test for seasonal unit roots in $\alpha(L)$ polynomial in (1). That is, the null hypothesis of interest is

$$
H_{0}: \alpha(z)=1-z^{S}=: \Delta_{S} .
$$


As detailed in Smith et al. (2009), the $S$ th order polynomial $a(L)$ can be factorised as $\alpha(L)=$ $\prod_{k=0}^{\lfloor S / 2\rfloor} \omega_{k}(L),\lfloor\cdot\rfloor$ denoting the integer part of its argument, where: $\omega_{0}(L):=\left(1-\alpha_{0} L\right)$ associates the parameter $\alpha_{0}$ with the zero frequency $\omega_{0}:=0, \omega_{k}(L):=\left[1-2\left(\alpha_{k} \cos \omega_{k}-\beta_{k} \sin \omega_{k}\right) L+\left(\alpha_{k}^{2}+\beta_{k}^{2}\right) L^{2}\right]$ corresponds to the conjugate (harmonic) seasonal frequencies $\left(\omega_{k}, 2 \pi-\omega_{k}\right), \omega_{k}=2 \pi k / S$, with the associated parameters $\alpha_{k}$ and $\beta_{k}, k=1, \ldots, S^{*}, S^{*}:=\lfloor(S-1) / 2\rfloor$, and, for $S$ even, $\omega_{S / 2}(L):=$ $\left(1+\alpha_{S / 2} L\right)$ associates the parameter $\alpha_{S / 2}$ with the Nyquist frequency $\omega_{S / 2}:=\pi .^{2}$ The null hypothesis in (4) can therefore be partitioned as $H_{0}=\cap_{k=0}^{\lfloor S / 2\rfloor} H_{0, k}$, where

$$
\begin{aligned}
& H_{0,0}: \alpha_{0}=1, H_{0, S / 2}: \alpha_{S / 2}=1, \\
& H_{0, k}: \alpha_{k}=1, \beta_{k}=0, k=1, \ldots, S^{*} .
\end{aligned}
$$

The hypothesis $H_{0,0}$ corresponds to a unit root at the zero frequency, $H_{0, S / 2}$ corresponds to a unit root at the Nyquist frequency, while $H_{0, k}$ corresponds to a pair of complex conjugate unit roots at the $k$-th harmonic seasonal frequency pair. The alternative hypothesis of stationarity at one or more of the zero or seasonal frequencies is given by $H_{1}=\cup_{k=0}^{\lfloor S / 2\rfloor} H_{1, k}$, where

$$
\begin{aligned}
H_{1,0}:\left|\alpha_{0}\right| & <1, H_{1, S / 2}:\left|\alpha_{S / 2}\right|<1, \\
H_{1, k}: \alpha_{k}^{2}+\beta_{k}^{2} & <1, k=1, \ldots, S^{*} .
\end{aligned}
$$

Cf. Smith et al. (2009).

\subsection{Augmented HEGY Tests}

Expanding the composite $A R(p+S)$ polynomial $\phi^{*}(z):=\alpha(z) \phi(z)$ around the zero and seasonal frequency unit roots $\exp ( \pm i 2 \pi k / S), k=0, \ldots,\lfloor S / 2\rfloor$, we obtain the auxiliary HEGY regression,

$$
\begin{aligned}
\Delta_{S} x_{S n+s}=\pi_{0} x_{0, S n+s-1}+\pi_{S / 2} x_{S / 2, S n+s-1}+\sum_{k=1}^{S^{*}}\left(\pi_{\alpha, k} x_{k, S n+s-1}^{\alpha}\right. & \left.+\pi_{\beta, k} x_{k, S n+s-1}^{\beta}\right) \\
& +\sum_{j=1}^{p} \phi_{j}^{*} \Delta_{S} x_{S n+s-j}+\varepsilon_{S n+s}
\end{aligned}
$$

where the regressors are defined as, $x_{0, S n+s}:=\sum_{j=0}^{S-1} x_{S n+s-j}, x_{S / 2, S n+s}:=\sum_{j=0}^{S-1} \cos [(j+1) \pi] x_{S n+s-j}$, and $x_{k, S n+s}^{\alpha}:=\sum_{j=0}^{S-1} \cos \left[(j+1) \omega_{k}\right] x_{S n+s-j}$, and $x_{k, S n+s}^{\beta}:=-\sum_{j=0}^{S-1} \sin \left[(j+1) \omega_{k}\right] x_{S n+s-j}$, in each case for $k=1, \ldots, S^{*}$; cf. Proposition 1 of Smith et al. (2009, p.533). Notice that (9) is an unrestricted re-parameterisation of (1)-(2). In what follows we assume that the practitioner has available the sample observations $\left\{x_{1}, \ldots x_{T}\right\}$ so that (9) may be treated as being estimated along the single index $t=S+1+p, \ldots, T$.

Unit roots at the zero, Nyquist and harmonic seasonal frequencies imply that $\pi_{0}=0, \pi_{S / 2}=0$ and $\pi_{\alpha, k}=\pi_{\beta, k}=0, k=1, \ldots, S^{*}$, respectively, in (9); see Smith et al. (2009). Consequently, tests for

\footnotetext{
${ }^{2}$ In what follows, it is understood that terms relating to frequency $\pi$ are to be omitted when $S$ is odd and that where reference is made to the Nyquist frequency this is understood only to apply where $S$ is even.
} 
the presence or otherwise of a unit root at the zero and Nyquist frequencies are conventional lower-tail $t$-tests, denoted by $t_{0}$ and $t_{S / 2}$ for the exclusion of $x_{0, S n+s-1}$ and $x_{S / 2, S n+s-1}$, respectively, from (9). Similarly, the hypothesis of a pair of complex unit roots at the $k$ th harmonic seasonal frequency may be tested by the (upper-tailed) regression $F$-test, denoted $F_{k}$, for the exclusion of both $x_{k, S n+s-1}^{\alpha}$ and $x_{k, S n+s-1}^{\beta}$ from (9). Ghysels et al. (1994) also consider the joint frequency (upper-tail) regression $F$-tests from (9), $F_{1 \ldots\lfloor S / 2\rfloor}$ for the exclusion of $x_{S / 2, S n+s-1},\left\{x_{j, S n+s-1}^{\alpha}\right\}_{j=1}^{S^{*}}$ and $\left\{x_{j, S n+s-1}^{\beta}\right\}_{j=1}^{S^{*}}$, and $F_{0 \ldots\lfloor S / 2\rfloor}$ for the exclusion of $x_{0, S n+s-1}, x_{S / 2, S n+s-1},\left\{x_{j, S n+s-1}^{\alpha}\right\}_{j=1}^{S^{*}}$ and $\left\{x_{j, S n+s-1}^{\beta}\right\}_{j=1}^{S^{*}}$. The former tests the null hypothesis of unit roots at all of the seasonal frequencies, $\cap_{k=1}^{\lfloor S / 2\rfloor} H_{0, k}$, whereas the latter tests the overall null hypothesis, $H_{0}$ of (4). Implementation of these tests, including relevant critical values, has been considered in, inter alia, HEGY, Smith et al. (2009) and Ghysels et al. (1994).

In order to obtain representations for the asymptotic null distributions of the HEGY tests outlined above from (9) when the volatility process satisfies Assumption 3 we first rewrite the $x_{S n+s}$ in vectorof-seasons form, as is done in, inter alia, Burridge and Taylor (2001a,b), Smith et al. (2009), and del Barrio Castro et al. (2012) [hereafter, BCOT]. Under $H_{0}$ of (4), the vector-of-seasons representation for $x_{S n+s}$ is given by

$$
\mathbf{X}_{n}=\mathbf{X}_{n-1}+\mathbf{U}_{n}, n=2, \ldots, N,
$$

where we have defined $\mathbf{X}_{n}:=\left[x_{S n-(S-1)}, x_{S n-(S-2)}, \ldots, x_{S n}\right]^{\prime}$ and $\mathbf{U}_{n}:=\left[u_{S n-(S-1)}, u_{S n-(S-2)}, \ldots, u_{S n}\right]^{\prime}$, $n=1, \ldots, N$, and where the vector error process $\mathbf{U}_{n}$ satisfies the vector $\mathrm{MA}(\infty)$ representation

$$
\mathbf{U}_{n}=\sum_{j=0}^{\infty} \boldsymbol{\Psi}_{j} \mathcal{E}_{n-j},
$$

where the $(S \times S)$ matrices $\boldsymbol{\Psi}_{j}$ are defined as

$$
\mathbf{\Psi}_{0}:=\left[\begin{array}{cccccc}
1 & 0 & 0 & 0 & \ldots & 0 \\
\psi_{1} & 1 & 0 & 0 & \ldots & 0 \\
\psi_{2} & \psi_{1} & 1 & 0 & \ldots & 0 \\
\psi_{3} & \psi_{2} & \psi_{1} & 1 & \ldots & 0 \\
\vdots & \vdots & \vdots & \vdots & \ddots & \vdots \\
\psi_{S-1} & \psi_{S-2} & \psi_{S-3} & \psi_{S-4} & \ldots & 1
\end{array}\right]
$$

and

$$
\boldsymbol{\Psi}_{j}:=\left[\begin{array}{cccccc}
\psi_{j S} & \psi_{j S-1} & \psi_{j S-2} & \psi_{j S-3} & \ldots & \psi_{j S-(S-1)} \\
\psi_{j S+1} & \psi_{j S} & \psi_{j S-1} & \psi_{j S-2} & \ldots & \psi_{j S-(S-2)} \\
\psi_{j S+2} & \psi_{j S+1} & \psi_{j S} & \psi_{j S-1} & \ldots & \psi_{j S-(S-3)} \\
\psi_{j S+3} & \psi_{j S+2} & \psi_{j S+1} & \psi_{j S} & \ldots & \psi_{j S-(S-4)} \\
\vdots & \vdots & \vdots & \vdots & \ddots & \vdots \\
\psi_{j S+S-1} & \psi_{j S+S-2} & \psi_{j S+S-3} & \psi_{j S+S-4} & \ldots & \psi_{j S}
\end{array}\right], \quad j=1,2, \ldots
$$

where $\psi_{j}, j=1,2, \ldots$, are the MA coefficients from the inverse of $\psi(z)$; see Remark 3.

The following Lemma extends the multivariate invariance principle from Burridge and Taylor (2001a) to the case where the innovations display non-stationary volatility. 
Lemma 1 Let $\mathbf{X}_{n}$ be generated by (10)-(11). Then, under Assumptions 1 and 2 , in $\mathcal{D}_{\mathbb{R}^{m \times n}}[0,1]$

$$
N^{-1 / 2} \mathbf{X}_{\lfloor\cdot N\rfloor} \Rightarrow \boldsymbol{\Psi}(1) \mathbf{M}(\cdot)
$$

where " $\Rightarrow$ " denotes weak convergence as $N \rightarrow \infty, \mathbf{\Psi}(1):=\sum_{j=0}^{\infty} \boldsymbol{\Psi}_{j}$, and $\mathbf{M}(\cdot):=\int_{0}^{\cdot} \boldsymbol{\Omega}(u) d \mathbf{W}(u)$ is an $S$-variate continuous martingale, with $\mathbf{W}(\cdot):=\left(W_{1-S}(\cdot), \ldots, W_{0}(\cdot)\right)^{\prime}$ an $S$-dimensional standard Brownian motion process.

Remark 5 The result in Lemma 1 shows that in contrast to the form of $\mathrm{PH}$ considered in Burridge and Taylor (2001a) - where the seasonal variances can vary across seasons, but not across years the scaled vector-of-seasons data does not converge in the limit under the seasonal unit root null hypothesis to a vector Brownian motion; rather, it converges to a process with increments which, although still independent, are no longer identically distributed through time. More specifically, the limiting process $\mathbf{M}(\cdot)$ is a continuous martingale with spot volatility $\boldsymbol{\Omega}(\cdot)$ and integrated covariation equal to $\boldsymbol{\Sigma}(\cdot):=\int_{0}^{\cdot} \boldsymbol{\Omega}(u) \boldsymbol{\Omega}(u)^{\prime} d u$; cf. Shephard (2005).

Remark 6 Following the discussion in Cavaliere et al. (2010, Remark 2.6), the diagonality of $\boldsymbol{\Omega}_{n}$ implies that the limiting process $\mathbf{M}(\cdot)$ can be written as a vector variance-transformed Brownian motion on [0,1] with independent elements; see Davidson (1994, pp.486-492). In particular, in this case we have that $\left.\mathbf{M}(\cdot)=\left[\bar{\sigma}_{1-S} W_{\eta_{1-S}, 1-S}(\cdot)\right), \ldots, \bar{\sigma}_{0} W_{\eta_{0}, 0}(\cdot)\right]^{\prime}$, where $W_{\eta_{s}, s}(\cdot)=W_{s}\left(\eta_{s}(\cdot)\right)$, with $\eta_{s}(\cdot):=$ $\bar{\sigma}_{s}^{-2} \int_{0}^{\cdot} \sigma_{s}(r)^{2} d r, \bar{\sigma}_{s}:=\left(\int_{0}^{1} \sigma_{s}(r)^{2} d r\right)^{1 / 2}, s=1-S, \ldots, 0$, and where $\left\{W_{s}(\cdot)\right\}_{s=1-S}^{0}$, are the independent standard Brownian motions defined in Lemma 1. The limiting processes $\left\{W_{s}\left(\eta_{s}(\cdot)\right)\right\}_{s=1-S}^{0}$ are a set of $S$ (seasonally indexed) independent scalar variance-transformed Brownian motions (Brownian motions under a modification of the time domain) with directing processes $\eta_{s}(\cdot)$; see, inter alia, Cavaliere and Taylor (2007, p.924) for further discussion on variance transformed Brownian motions. For the form of $\mathrm{PH}$ considered in Burridge and Taylor (2001a), this simplifies further to $\mathbf{M}(\cdot)=$ $\left[\sigma_{1-S} W_{1-S}(\cdot), \ldots, \sigma_{0} W_{0}(\cdot)\right]^{\prime}$.

Using Lemma 1 we are now in a position to detail the asymptotic null distributions of the HEGY test statistics from (9) under the very general form of heteroskedasticity allowed under Assumption 1. These results are collected together in Proposition 1.

Proposition 1 Let the conditions of Lemma 1 hold. The statistics $t_{0}, t_{S / 2}, F_{k}, k=1, \ldots, S^{*}$, $F_{1 \ldots\lfloor S / 2\rfloor}$ and $F_{0 \ldots\lfloor S / 2\rfloor}$ then satisfy, as $N \rightarrow \infty$, the following weak convergence results:

$$
\begin{gathered}
t_{i} \Rightarrow \varsigma_{i}\left(\varphi_{i}\left\{c_{0}^{\prime} \boldsymbol{\Sigma}(1) c_{0}\right\} / S\right)^{-1 / 2}=: \xi_{\eta, i}, \quad i=0, S / 2, \\
F_{k} \Rightarrow \frac{S}{2 \varphi_{k}\left\{c_{0}^{\prime} \boldsymbol{\Sigma}(1) c_{0}\right\}}\left[\left(\varsigma_{k}^{\alpha}\right)^{2}+\left(\varsigma_{k}^{\beta}\right)^{2}\right]=: \xi_{\eta, k}, \quad k=1, \ldots, S^{*}, \\
F_{1 \ldots\lfloor S / 2\rfloor} \Rightarrow \frac{1}{S-1}\left(\xi_{\eta, S / 2}^{2}+2 \sum_{k=1}^{S^{*}} \xi_{\eta, k}\right)=: \xi_{\eta, 1 \ldots\lfloor S / 2\rfloor},
\end{gathered}
$$




$$
F_{0 \ldots\lfloor S / 2\rfloor} \Rightarrow \frac{1}{S}\left(\xi_{\eta, 0}^{2}+\xi_{\eta, S / 2}^{2}+2 \sum_{k=1}^{S^{*}} \xi_{\eta, k}\right)=: \xi_{\eta, 0 \ldots\lfloor S / 2\rfloor},
$$

where

$$
\begin{gathered}
\varsigma_{i}:=\frac{1}{S} c_{i}^{\prime} \mathbf{Q}_{1}^{*} c_{i}, i=0, S / 2, \\
\varsigma_{k}^{\alpha}:=\frac{1}{S}\left[c_{k}^{\prime} \mathbf{Q}_{1}^{*} c_{k}+\tilde{c}_{k}^{\prime} \mathbf{Q}_{1}^{*} \tilde{c}_{k}\right], \quad \varsigma_{k}^{\beta}:=\frac{1}{S}\left[c_{k}^{\prime} \mathbf{Q}_{1}^{*} \tilde{c}_{k}-\tilde{c}_{k}^{\prime} \mathbf{Q}_{1}^{*} c_{k}\right], k=1, \ldots, S^{*}, \\
\varphi_{i}:=\frac{1}{S} c_{i}^{\prime} \mathbf{Q}_{2}^{*} c_{i}, i=0, S / 2, \quad \varphi_{k}:=\frac{1}{2 S}\left[c_{k}^{\prime} \mathbf{Q}_{2}^{*} c_{k}+\tilde{c}_{k}^{\prime} \mathbf{Q}_{2}^{*} \tilde{c}_{k}\right], k=1, \ldots, S^{*}, \\
\mathbf{Q}_{1}^{*}:=\int_{0}^{1} \mathbf{M}(r) d \mathbf{M}(r)^{\prime}, \quad \mathbf{Q}_{2}^{*}:=\int_{0}^{1} \mathbf{M}(r) \mathbf{M}(r)^{\prime} d r
\end{gathered}
$$

and the (mutually orthogonal) selection vectors are defined as $c_{0}:=[1,1,1, \ldots, 1]^{\prime}, c_{S / 2}:=[1,-1,1,-1 \ldots, 1]^{\prime}$, $c_{k}:=\left[\cos \left(\omega_{k}[1-S]\right), \cos \left(\omega_{k}[2-S]\right), \ldots, \cos (0)\right]^{\prime}$ and $\tilde{c}_{k}:=\left[\sin \left(\omega_{k}[1-S]\right), \sin \left(\omega_{k}[2-S]\right), \ldots, \sin (0)\right]^{\prime}$, $k=1, \ldots, S^{*}$.

The following corollary of Proposition 1 gives alternative representations for the limiting null distributions of the HEGY statistics which appear therein. These representations are useful in that they can be naturally related back to existing large sample results in this literature. Some remarks about these limiting null distributions follow the corollary.

Corollary 1 Let the conditions of Lemma 1 hold. The statistics $t_{0}, t_{S / 2}, F_{k}, k=1, \ldots, S^{*}, F_{1 \ldots\lfloor S / 2\rfloor}$ and $F_{0 \ldots\lfloor S / 2\rfloor}$ then have the following asymptotic distributions:

$$
\begin{gathered}
t_{i} \Rightarrow \frac{\int_{0}^{1} B_{\eta, i}(r) d B_{\eta, i}(r)}{\sqrt{\int_{0}^{1} B_{\eta, i}^{2}(r) d r}}=: \xi_{\eta, i}, i=0, S / 2, \quad F_{k} \Rightarrow \frac{1}{2}\left[\left(\xi_{k}^{\alpha}\right)^{2}+\left(\xi_{k}^{\beta}\right)^{2}\right]=: \xi_{\eta, k}, k=1, \ldots, S^{*}, \\
F_{1 \ldots\lfloor S / 2\rfloor} \Rightarrow \frac{1}{S-1}\left(\xi_{\eta, S / 2}^{2}+2 \sum_{k=1}^{S^{*}} \xi_{\eta, k}\right)=: \xi_{\eta, 1 \ldots\lfloor S / 2\rfloor}, \quad F_{0 \ldots\lfloor S / 2\rfloor} \Rightarrow \frac{1}{S}\left(\xi_{\eta, 0}^{2}+\xi_{\eta, S / 2}^{2}+2 \sum_{k=1}^{S^{*}} \xi_{\eta, k}\right)=: \xi_{\eta, 0 \ldots\lfloor S / 2\rfloor},
\end{gathered}
$$

where

$$
\begin{aligned}
\xi_{k}^{\alpha} & :=\frac{\frac{1}{S}\left\{c_{k}^{\prime} \boldsymbol{\Sigma}(1) c_{k}\right\} \int_{0}^{1} B_{\eta, k}(r) d B_{\eta, k}(r)+\frac{1}{S}\left\{\tilde{c}_{k}^{\prime} \boldsymbol{\Sigma}(1) \tilde{c}_{k}\right\} \int_{0}^{1} B_{\eta, k}^{*}(r) d B_{\eta, k}^{*}(r)}{\left[\frac{1}{2 S}\left\{c_{k}^{\prime} \boldsymbol{\Sigma}(1) c_{k}\right\} \int_{0}^{1} B_{\eta, k}^{2}(r) d r+\frac{1}{2 S}\left\{\tilde{c}_{k}^{\prime} \boldsymbol{\Sigma}(1) \tilde{c}_{k}\right\} \int_{0}^{1} B_{\eta, k}^{* 2}(r) d r\right]^{1 / 2}\left[\left\{c_{0}^{\prime} \boldsymbol{\Sigma}(1) c_{0}\right\} / S\right]^{1 / 2}}, \quad k=1, \ldots, S^{*}, \\
\xi_{k}^{\beta} & :=\frac{\frac{1}{S}\left\{c_{k}^{\prime} \boldsymbol{\Sigma}(1) c_{k}\right\}^{1 / 2}\left\{\tilde{c}_{k}^{\prime} \boldsymbol{\Sigma}(1) \tilde{c}_{k}\right\}^{1 / 2}\left[\int_{0}^{1} B_{\eta, k}(r) d B_{\eta, k}^{*}(r)-\int_{0}^{1} B_{\eta, k}^{*}(r) d B_{\eta, k}(r)\right]}{\left[\frac{1}{2 S}\left\{c_{k}^{\prime} \boldsymbol{\Sigma}(1) c_{k}\right\} \int_{0}^{1} B_{\eta, k}^{2}(r) d r+\frac{1}{2 S}\left\{\tilde{c}_{k}^{\prime} \boldsymbol{\Sigma}(1) \tilde{c}_{k}\right\} \int_{0}^{1} B_{\eta, k}^{* 2}(r) d r\right]^{1 / 2}\left[\left\{c_{0}^{\prime} \boldsymbol{\Sigma}(1) c_{0}\right\} / S\right]^{1 / 2}}, \quad k=1, \ldots, S^{*},
\end{aligned}
$$

and where

$$
\begin{gathered}
B_{\eta, i}(\cdot):=\left(c_{i}^{\prime} \boldsymbol{\Sigma}(1) c_{i}\right)^{-1 / 2} c_{i}^{\prime} \mathbf{M}(\cdot), i=0, S / 2, \\
B_{\eta, k}(\cdot):=\left(c_{k}^{\prime} \boldsymbol{\Sigma}(1) c_{k}\right)^{-1 / 2} c_{k}^{\prime} \mathbf{M}(\cdot), B_{\eta, k}^{*}(\cdot):=\left(\tilde{c}_{k}^{\prime} \boldsymbol{\Sigma}(1) \tilde{c}_{k}\right)^{-1 / 2} \tilde{c}_{k}^{\prime} \mathbf{M}(\cdot), k=1, \ldots, S^{*}
\end{gathered}
$$

are a set of $S$ mutually independent (normalised) variance-transformed Brownian motion processes obtained via the (mutually orthogonal) selection vectors $c_{0}:=[1,1,1, \ldots, 1]^{\prime}, c_{S / 2}:=[1,-1,1,-1 \ldots, 1]^{\prime}$, $c_{k}:=\left[\cos \left(\omega_{k}[1-S]\right), \cos \left(\omega_{k}[2-S]\right), \ldots, \cos (0)\right]^{\prime}$ and $\tilde{c}_{k}:=\left[\sin \left(\omega_{k}[1-S]\right), \sin \left(\omega_{k}[2-S]\right), \ldots, \sin (0)\right]^{\prime}$, $k=1, \ldots, S^{*}$. 
Remark 7 The representations given in Proposition 1 for the limiting null distributions of the $t_{0}$ and $t_{S / 2}$ statistics are mutually independent and independent of those for $F_{k}, k=1, \ldots, S^{*}$, which are also mutually independent across $k=1, \ldots, S^{*}$. For given variance profiles, the representations for the $t_{0}$ and $t_{S / 2}$ statistics have the same functional form as those for the limiting null distribution of the non-seasonal $\mathcal{M Z}_{t}$ statistic (which coincides with that of the familiar augmented Dickey-Fuller statistic) in Theorem 1 of Cavaliere and Taylor (2007, p.924) for the case where no detrending is performed. This equivalence also holds when detrending is performed; see Remark 11. The limiting null distributions of the $t_{0}, t_{S / 2}, F_{k}, k=1, \ldots, S^{*}, F_{1 \ldots\lfloor S / 2\rfloor}$ and $F_{0 \ldots\lfloor S / 2\rfloor}$ statistics are also seen to be free of any weak dependence nuisance parameters arising from the stationary lag polynomial $\phi(z)$.

Remark 8 The variance profile, $\eta^{0}(r)$ say, which characterises the zero frequency variance transformed Brownian motion $B_{\eta, 0}(r)$ in the representation for the limiting null distribution of the $t_{0}$ statistic in Proposition 1 can be seen to constitute the average limit taken across $s=1-S, \ldots, 0$ of the finite sample analogues, say $\eta_{N, s}(r):=\left(N^{-1} \sum_{n=1}^{\lfloor r N\rfloor} \sigma_{S n+s}^{2}\right) /\left(N^{-1} \sum_{n=1}^{N} \sigma_{S n+s}^{2}\right)$, of the seasonal variance profiles, $\eta_{s}(r), s=1-S, \ldots, 0$, which characterise the seasonally identified variance transformed Brownian motions $W_{\eta_{s}, s}(r)=W_{s}\left(\eta_{s}(r)\right), s=1-S, \ldots, 0$, defined in Remark 6. Consequently, changes in the seasonal variances which are smoothed out in large samples by taking the average over $S$ consecutive observations will not affect the limiting null distributions of the $t_{0}$ statistic vis-à-vis the homoskedastic case. An example of this occurs where $\sigma_{S n+s}=a_{s}, s=1-S, \ldots, 0$ for $n=1, \ldots,\lfloor\tau N\rfloor-1$, $\tau \in(0,1)$, but then undergoes a one-time break in each season at $n=\lfloor\tau N\rfloor$, switching to $\sigma_{S n+s}=b_{s}$, $s=1-S, \ldots, 0, n=\lfloor\tau N\rfloor, \ldots, N$, but does so such that $\sum_{s=1-S}^{0}\left(a_{s}^{2}-b_{s}^{2}\right)=0$. The same holds for the $t_{S / 2}$ statistic, but not for the $F_{k}, k=1, \ldots, S^{*}, F_{1 \ldots\lfloor S / 2\rfloor}$ and $F_{0 \ldots\lfloor S / 2\rfloor}$ statistics.

Remark 9 For the form of PH where $\boldsymbol{\Omega}_{n}=\operatorname{diag}\left\{\sigma_{1-S}, \ldots, \sigma_{0}\right\}$, the limiting distributions given in Proposition 1 reduce, for the quarterly case $S=4$, to those given in Corollary 3.1 of Burridge and Taylor (2001a). Under this form of PH the average of the variances of the innovations when taken over any $S$ consecutive observations is constant through the sample. It therefore follows from the discussion in Remark 8 that $t_{0}$ and $t_{S / 2}$ have standard Dickey-Fuller limiting null distributions in this case; that is, $t_{i} \Rightarrow\left(\int_{0}^{1} B_{i} d B_{i}\right) /\left(\int_{0}^{1} B_{i}^{2} d r\right)^{1 / 2}, i=0, S / 2$, with $B_{i}(r), i=0, S / 2$, mutually independent standard Brownian motions. In the unconditionally homoskedastic case, where $\boldsymbol{\Omega}_{n}=\sigma I_{S}$, the representations given in Proposition 1 simplify to those given in Theorem 1 of Smith et al. (2009). Neither Burridge and Taylor (2001a) nor Smith et al. (2009) allow for conditional heteroskedasticity in $\varepsilon_{t}$.

Remark 10 The results stated in Proposition 1 pertain to the seasonal unit root null hypothesis, $H_{0}$ of (4). Corresponding results under local alternatives of the form considered in Rodrigues and Taylor (2007), where the parameters in the decomposition of $a(L)$ given in section 3.1 are such that $a_{0}=$ $\left(1+\nu_{0} / T\right), a_{S / 2}=\left(1+\nu_{S / 2} / T\right), a_{k}=\left(1+\nu_{k} / T\right)$ and $\beta_{k}=0, k=1, \ldots, S^{*}$, with $\nu_{0}, \nu_{1}, \ldots, \nu_{\lfloor S / 2\rfloor}$ finite constants, can be obtained in similar fashion. In this case, the result in Lemma 1 holds on replacing the elements of the vector process $\mathbf{M}(r), W_{\eta_{s}, s}(r), s=1-S, \ldots, 0$, by the corresponding diffusion process $W_{\eta_{s}, c_{s}, s}(r):=\int_{0}^{r} \exp \left(-c_{s}(r-\lambda)\right) d W_{\eta_{s}, s}(\lambda), s=1-S, \ldots, 0$, where the season specific non-centrality 
parameters, $c_{s}, s=1-S, \ldots, 0$, are derived from the $\nu_{k}, k=0, \ldots,\lfloor S / 2\rfloor$ frequency specific noncentrality parameters. The representations given for the HEGY statistics in Theorem 5.1 of Rodrigues and Taylor (2007) can then be shown to hold on replacing the standard Ornstein-Uhlenbeck processes which feature there with the corresponding variance-transformed Ornstein-Uhlenbeck processes (which are characterised by the same variance profiles as under $H_{0}$ ) with non-centrality parameters $c_{0}, c_{S / 2}$ and $c_{k}, k=1, \ldots, S^{*}$. As a consequence, both the asymptotic size and local power functions of the HEGY tests are in general affected by non-constant volatility of the form considered in this paper (an exception being the $t_{0}$ and $t_{S / 2}$ tests in the $\mathrm{PH}$ case considered in Burridge and Taylor, 2001a).

Remark 11 Thus far we have considered the case where the process $\left\{x_{S n+s}\right\}$ admits no deterministic component. It is straightforward to extend the foregoing results to the case where the series contains deterministic elements. To that end, consider the following generalisation of the DGP in (1)-(3):

$$
y_{S n+s}=\mu_{S n+s}+x_{S n+s}, \quad s=1-S, \ldots, 0, \quad n=1, \ldots, N,
$$

with $x_{S n+s}$ in (13) as previously defined in (1)-(3) and where $\mu_{S n+s}:=\gamma^{\prime} Z_{S n+s}$ where $Z_{S n+s}$ is purely deterministic. Smith et al. (2009) present a typology of six cases of interest for $\mu_{S n+s}$, namely: no deterministic component (as considered above); non-seasonal intercept; non-seasonal intercept and non-seasonal trend; seasonal intercepts; seasonal intercepts and non-seasonal trend, and seasonal intercepts and seasonal trends. In order to yield tests which will be exact invariant (assuming $\mu_{S n+s}$ is not under-specified) to the elements of $\gamma$ which characterise the deterministic component $\mu_{S n+s}$, the HEGY regression in (9) must be constructed from appropriately de-trended data. This can either be done using OLS de-trending, as in, for example, HEGY and Smith et al. (2009), or by local GLS de-trending as in Rodrigues and Taylor (2007). Where a deterministic component is allowed for, define the resulting de-trended data series as $\hat{x}_{S n+s}:=x_{S n+s}-\hat{\gamma}^{\prime} Z_{S n+s}$, the HEGY regression variables in (9) are then constructed as before but from $\hat{x}_{S n+s}$ rather than $x_{S n+s}$. It is important to notice, as shown in Smith et al. (2009), that allowing for seasonal intercepts renders the resulting unit root tests exact similar with respect to the initial conditions, $x_{1}, \ldots, x_{S}$. Where $(9)$ is based on de-trended data, the results given in this section still hold provided the variance transformed standard Brownian motions, $B_{\eta, 0}(\cdot), B_{\eta, S / 2}(\cdot)$, and $B_{\eta, k}(\cdot)$ and $B_{\eta, k}^{*}(\cdot), k=1, \ldots, S^{*}$, are re-defined as appropriate to the deterministic scenario of interest; cf. Sections 4.1-4.5 of Smith and Taylor (1998) for OLS detrending and Theorem 5.1 of Rodrigues and Taylor (2007, pp.559-560) for local GLS de-trending. As an example, if de-trending is performed with respect to seasonal intercepts only then the variance transformed standard Brownian motions above are all replaced by their demeaned analogues, so that (for instance) $B_{\eta, 0}(r)$ is replaced by the process $B_{\eta, 0}-\int_{0}^{1} B_{\eta, 0}(u) d u$. The results discussed in Remarks 7-10 also remain apposite, mutatis mutandis.

\section{Wild Bootstrap HEGY Tests}

As demonstrated in Proposition 1, heteroskedasticity of the form given under Assumption introduces a time deformation aspect to the limiting distributions of the HEGY unit root statistics which alters 
their form vis-à-vis the homoskedastic case. Consequently, inference based on these statistics will not be correctly sized (even asymptotically) if standard (homoskedastic) critical values are used. In this section we propose wild bootstrap implementations of the HEGY tests and demonstrate that this allows us to retrieve (asymptotically) correct $p$-values in the presence of both heteroskedasticity of the form given in Assumption 1 and weak dependence in the shocks. Two possible, asymptotically equivalent, wild bootstrap re-sampling devices are proposed.

\subsection{The Seasonal Wild Bootstrap Algorithm}

We first outline our proposed algorithm which draws on the wild bootstrap principle; see, inter alia, $\mathrm{Wu}(1986)$.

\section{Algorithm 1 (Wild Bootstrap HEGY Tests)}

Step 1: Obtain the standard HEGY test statistics, $t_{0}, t_{S / 2}, F_{j}, j=1, \ldots, S^{*}, F_{1 \ldots\lfloor S / 2\rfloor}$ and $F_{0 \ldots\lfloor S / 2\rfloor}$, along with the corresponding OLS residuals, $\hat{\varepsilon}_{S n+s}, S n+s=S+p+$ $1, \ldots, T$, from estimating the HEGY regression (9), constructed from either $x_{S n+s}$ in the case where no deterministic component is allowed for, or $\hat{x}_{S n+s}$ where either OLS or local GLS de-trending is employed to allow for the deterministic component $\mu_{S n+s}:=\gamma^{\prime} Z_{S n+s}$; see Remark 11. Set $\hat{\varepsilon}_{S n+s}=0$ for $S n+s=1, \ldots, S+p$, and define the annual residual vectors $\hat{\mathbf{E}}_{n}:=\left[\hat{\varepsilon}_{S n-(S-1)}, \hat{\varepsilon}_{S n-(S-2)}, \ldots, \hat{\varepsilon}_{S n}\right]^{\prime}, n=1, \ldots, N$.

\section{Either}

Step 2a: Generate the vectors of wild bootstrap errors $\mathbf{E}_{n}^{*}:=\left[\varepsilon_{S n-(S-1)}^{*}, \varepsilon_{S n-(S-2)}^{*}, \ldots, \varepsilon_{S n}^{*}\right]^{\prime}$, whose elements are formed using a randomisation device of the form $\varepsilon_{S n+s}^{*}:=\hat{\varepsilon}_{S n+s} w_{S n+s}$, $S n+s=1, \ldots, T$, where $\left\{w_{S n+s}\right\}_{S n+s=1}^{T}$ denotes an i.i.d. sequence with $E\left(w_{S n+s}\right)=$ $0, E\left(w_{S n+s}^{2}\right)=1$ and $E\left(w_{S n+s}^{4}\right)<\infty$.

Or

Step 2b: Generate $\mathbf{E}_{n}^{*}$ according to the device $\mathbf{E}_{n}^{*}=\hat{\mathbf{E}}_{n} w_{n}$, where $\left\{w_{n}\right\}_{n=1}^{N}$ denotes an i.i.d. sequence with $E\left(w_{n}\right)=0, E\left(w_{n}^{2}\right)=1$ and $E\left(w_{n}^{4}\right)<\infty$.

Step 3: Construct the bootstrap sample data through the recursion

$$
\Delta_{S} x_{S n+s}^{*}=\varepsilon_{S n+s}^{*}, n=2, \ldots, N, s=1-S, \ldots, 0,
$$

initialized at $y_{1}^{*}=\cdots=y_{S}^{*}=0$.

Step 4: Using the bootstrap sample, $\left\{x_{S n+s}^{*}\right\}$, compute the bootstrap HEGY statistics, denoted $t_{0}^{*}, t_{S / 2}^{*}, F_{j}^{*}, j=1, \ldots, S^{*}, F_{1 \ldots\lfloor S / 2\rfloor}^{*}$ and $F_{0 \ldots\lfloor S / 2\rfloor}^{*}$ with an obvious notation, exactly as was done for the original data in Step 1 (including any de-trending), for some fixed lag length $p^{*} \geq 0$ in (9).

Step 5: Bootstrap p-values are then defined as: $P_{j, T}^{*}:=G_{j, T}^{*}\left(t_{j}\right), j=0, S / 2, P_{j, T}^{*}:=1-$ $G_{j, T}^{*}\left(F_{j}\right), j=1, \ldots, S^{*}, P_{1 \ldots\lfloor S / 2\rfloor, T}^{*}:=1-G_{1 \ldots\lfloor S / 2\rfloor, T}^{*}\left(F_{1 \ldots\lfloor S / 2\rfloor}\right)$ and $P_{0 \ldots\lfloor S / 2\rfloor, T}^{*}:=1-$ 
$G_{0 \ldots\lfloor S / 2\rfloor, T}^{*}\left(F_{0 \ldots\lfloor S / 2\rfloor}\right)$, where $G_{k, T}^{*}(\cdot), k=0, \ldots,\lfloor S / 2\rfloor, G_{1 \ldots\lfloor S / 2\rfloor, T}^{*}(\cdot)$ and $G_{0 \ldots\lfloor S / 2\rfloor, T}^{*}(\cdot)$ denote the conditional (on the original sample data) cumulative distribution functions (cdf's) of $t_{0}^{*}, F_{j}^{*}, j=1, \ldots, S^{*}, t_{S / 2}^{*}, F_{1 \ldots\lfloor S / 2\rfloor}^{*}$ and $F_{0 \ldots\lfloor S / 2\rfloor}^{*}$, respectively. In practice, the cdf's required here will be unknown, but can be approximated in the usual way through numerical simulation.

Remark 12 Step 2a of Algorithm 1 employs a standard wild bootstrap re-sampling scheme, whereby each residual from the estimated HEGY regression is multiplied by a different external random variable $w_{S n+s}$. In contrast, Step $2 \mathrm{~b}$ employs a seasonal block wild bootstrap, whereby the same randomisation device is employed for each season within a given year; that is, all of the residuals within year $n$ are multiplied by the same external scalar random variable, $w_{n} \cdot{ }^{3}$ As we will show below these two schemes are asymptotically equivalent. Their finite sample properties will be explored in section 5 .

Remark 13 In Step 2 of Algorithm 1 it is the multiplicative factor, either $w_{S n+s}$ in Step 2 a or $w_{n}$ in Step $2 b$, that distinguishes the wild bootstrap HEGY tests we propose here from the corresponding i.i.d. bootstrap HEGY tests of Burridge and Taylor (2014) and Zuo and Politis (2016), and from the block bootstrap HEGY tests of Zuo and Politis (2016). This multiplicative factor serves to replicate the pattern of heteroskedasticity present in the seasonal innovations in the bootstrap errors because, conditionally on the original data, $\varepsilon_{S n+s}^{*}$ has zero mean and variance $\hat{\varepsilon}_{S n+s}^{2}$. This would not be achieved by using either the i.i.d. or block bootstrap approaches mentioned above.

\subsection{Asymptotic Properties}

In this section we discuss the asymptotic properties of the wild bootstrap HEGY unit root tests. We show that under $H_{0}$ of (4), and for any error process $u_{t}$ satisfying Assumptions 1 and 2 , the bootstrap HEGY statistics converge to the same asymptotic distributions as their counterpart test statistics computed on the original data; i.e. our proposed wild bootstrap allows us to replicate the correct first-order asymptotic null distributions of each of the HEGY statistics. This holds regardless of whether Step 2a or Step 2b of Algorithm 1 is used. We also discuss the asymptotic properties of the bootstrap HEGY statistics under both near-integrated and stable autoregressive alternatives.

The usefulness of the wild bootstrap in the present framework is given in the following result, which shows that it allows us to retrieve the correct asymptotic null distributions of the HEGY tests and, hence, that the $p$-values from Step 5 of Algorithm 1 are all asymptotically pivotal.

Proposition 2 Let the conditions of Lemma 1 hold. Then $t_{i}^{*} \Rightarrow_{p} \xi_{\eta, i}, i=0, S / 2, F_{k}^{*} \Rightarrow_{p} \xi_{\eta, k}$, $k=1, \ldots, S^{*}, F_{1 \ldots\lfloor S / 2\rfloor}^{*} \Rightarrow_{p} \xi_{\eta, 1 \ldots\lfloor S / 2\rfloor}$, and $F_{0 \ldots\lfloor S / 2\rfloor}^{*} \Rightarrow_{p} \xi_{\eta, 0 \ldots\lfloor S / 2\rfloor}$, where $\Rightarrow_{p}$ is used to denote weak convergence in probability in the sense of Giné and Zinn (1990). Moreover, $P_{T}^{*} \stackrel{w}{\rightarrow} U[0,1]$, where $P_{T}^{*}$ is

\footnotetext{
${ }^{3}$ It should be noted that the idea of a block wild bootstrap is not new. Shao (2011) proposes a block wild bootstrap in the context of bootstrap tests for white noise. However, the block wild bootstrap scheme in Step $2 b$ differs from that in Shao (2011) in the important regard that while our block length is fixed and equal to the number of seasons, in Shao (2011) the block length is an increasing function of the sample size.
} 
again used generically to denote any of the HEGY p-values defined in Algorithm 1, and $U[0,1]$ denotes a uniform distribution on $[0,1]$.

Remark 14 An immediate implication of Proposition 2 is that the wild bootstrap HEGY tests will all be asymptotically correctly sized under Assumption 1, with this result holding regardless of whether Step 2a or Step 2b of Algorithm 1 is used. This follows on noting that the stated results imply that for each of the bootstrap HEGY tests, with $u \in(0,1)$ denoting the chosen nominal level, under the null hypothesis and as $T \rightarrow \infty$ it holds that $P\left(P_{T}^{*} \leq u\right)=u$, implying that the tests are asymptotically size controlled. Notice that the results in Proposition 2 are trivially also seen to be true under conditional homoskedasticity because that special case is contained within Assumption 1.

Remark 15 Because the HEGY regression in (9) is an unrestricted re-parameterisation of (1)-(2) it is straightforward to show that the limiting distributions given for the wild bootstrap HEGY $t_{i}^{*}$, $i=0, S / 2, F_{k}^{*}, k=1, \ldots, S^{*}, F_{1 \ldots\lfloor S / 2\rfloor}^{*}$, and $F_{0 \ldots\lfloor S / 2\rfloor}^{*}$ statistics in Proposition 2 also hold under both local alternatives of the form given in Remark 10 and also under fixed alternatives of the form given in (7)-(8). An immediate consequence of the first of these results is that under Assumption 1, the wild bootstrap HEGY tests will attain the same asymptotic local power function as the size-adjusted HEGY tests. The second result implies that the bootstrap HEGY tests share the same consistency properties as the original HEGY tests. In particular, under the fixed alternative $H_{1, j}$ in $(7), t_{j}$ diverges to minus infinity at rate $O_{p}\left(N^{1 / 2}\right), j=0, S / 2$, while under $H_{1, k}$ in $(8), F_{j}, j=1, \ldots, S^{*}$, diverges to positive infinity at rate $O_{p}(N)$. Finally, under $\cup_{k=j}^{\lfloor S / 2\rfloor} H_{1, k}, F_{j \ldots\lfloor S / 2\rfloor}, j=0,1$, diverges to positive infinity at rate $O_{p}(N)$. These rates are unaltered under Assumption 1 vis-à-vis the usual homoskedastic case. The asymptotic theory therefore predicts that the bootstrap HEGY tests should have finite sample power approximately equal to the size-adjusted power of the standard HEGY tests.

\section{$5 \quad$ Finite Sample Simulations}

We now investigate the finite sample size and power properties of the HEGY tests from section 3.2 and the corresponding wild bootstrap HEGY tests from section 4 when the shocks display a variety of forms of heteroskedasticity permitted under Assumption 1. Results are reported and discussed in the main text for wild bootstrap HEGY tests based on using Step 2a of Algorithm 1. ${ }^{4}$ All of the reported results pertain to the case where the data are de-trended (results are reported for both OLS and local GLS de-trended data) as outlined in Remark 11 allowing for seasonal intercepts and a non-seasonal trend. All experiments were based on 10,000 Monte Carlo replications. For the wild bootstrap HEGY tests, $B=499$ bootstrap replications were used with the $w_{S n+s}$ variables used in step 2 set as independent standard normal variables (independent across both the sample data points and across bootstrap replications). For the standard HEGY tests, conventional asymptotic critical

\footnotetext{
${ }^{4}$ Corresponding results for the wild bootstrap HEGY tests using the seasonal block wild re-sampling device in Step $2 \mathrm{~b}$ of Algorithm 1 can be found in the accompanying on-line supplementary appendix. A comparison of these results suggests that overall the two wild bootstrap schemes give qualitatively very similar finite sample behaviour.
} 
values (based on the assumption of homoskedasticity) were used. All simulations were programmed in Ox 7.0 using the rann random number generator.

The Monte Carlo simulations reported in this section are based on data generated by the quarterly $(S=4)$ DGP:

$$
\begin{aligned}
\alpha(L) x_{4 n+s} & =u_{4 n+s}, s=-3, \ldots, 0, n=2, \ldots, N, \\
\phi(L) u_{4 n+s} & =\theta(L) \varepsilon_{4 n+s} \\
\varepsilon_{4 n+s} & =\sigma_{4 n+s} e_{4 n+s}, e_{4 n+s} \sim \operatorname{NIID}(0,1)
\end{aligned}
$$

with $x_{1}=\cdots=x_{4}=0$ and $\varepsilon_{4 n+s}=0$ for $n \leq 0$. Data were generated from this $\mathrm{DGP}^{5}$ for samples of $N=50$ and $N=100$ years of data, with the volatility process $\sigma_{4 n+s}$ satisfying one of the following Models, where we define the notation $\Sigma_{i}:=\left(\sigma_{i,-3}, \sigma_{i,-2}, \sigma_{i,-1}, \sigma_{i, 0}\right), i=0,1$ :

- Model 1. Constant Unconditional Volatility: $\sigma_{S n+s}^{2}=\sigma_{0}^{2}, \sigma_{0}=1$.

- Model 2. Periodic Heteroskedasticity: $\sigma_{4 n+s}^{2}=\sigma_{0, s}^{2}$, with either: Case 1, $\Sigma_{0}=(3,1,3,1)$; or Case $2, \Sigma_{0}=(30,1,1,1)$.

- Model 3. Single Volatility Shift: $\sigma_{4 n+s}^{2}=\sigma_{0}^{2}+\left(\sigma_{1}^{2}-\sigma_{0}^{2}\right) \mathbb{I}(4 n+s \geq\lfloor\tau T\rfloor)$, with $\delta:=\sigma_{0} / \sigma_{1}$, $\sigma_{0}=1$.

- Model 4. Single Periodic Volatility Shift: $\sigma_{4 n+s}^{2}=\sigma_{0, s}^{2}+\left(\sigma_{1, s}^{2}-\sigma_{0, s}^{2}\right) \mathbb{I}(4 n+s \geq\lfloor\tau T\rfloor)$, with either: Case 1, $\left\{\Sigma_{0}=(3,1,3,1), \Sigma_{1}=\Sigma_{0} / \delta\right\}$; Case $2,\left\{\Sigma_{0}=(30,1,1,1), \Sigma_{1}=\Sigma_{0} / \delta\right\}$; Case 3: $\left\{\Sigma_{0}=(1,1,1,1), \Sigma_{1}=(1,1 / \delta, 1,1 / \delta)\right\} ;$ or Case $4,\left\{\Sigma_{0}=(3,1,3,1), \Sigma_{1}=(1,3,1,3)\right\}$.

In the case of Models 3 and 4 results are reported for $\delta \in\{1 / 3,3\}$ and $\tau \in\{0.2,0.8\}$. Notice that Model 4 combines the single volatility shift of Model 3, used in the non-seasonal case by Cavaliere and Taylor (2008a)), with the PH of Model 2, used in Burridge and Taylor (2001a), such that the relative pattern of $\mathrm{PH}$ between the seasons changes at the break-fraction $\tau .{ }^{6}$ Notice that under Case 3 of Model 4 the innovations do not display PH before the break but do afterwards. In Cases 1 and 2 of Model 4 the relative magnitude of the PH changes at the breakpoint, while in Cases 3 and 4 the pattern of PH changes at the breakpoint; for example, under Case 3 the innovations do not display $\mathrm{PH}$ before the break but do afterwards. Model 1, the homoskedastic case, provides a benchmark to compare the finite sample size and properties of the original HEGY tests and their wild bootstrap counterparts when no heteroskedasticity is in fact present. Notice finally that there is no seasonal aspect to the volatility process under Models 1 and 3 .

In practice the lag order $p$ in (9) is unknown. Consequently, all of the results which we report pertain to the case where a data-based method to estimate $p$ is used. Denoting this estimated lag

\footnotetext{
${ }^{5}$ We also considered a variety of conditionally heteroskedastic specifications for $e_{4 n+s}$, including stationary GARCH and autoregressive stochastic volatility models. Consistent with the findings of Cavaliere and Taylor (2009), the results for the wild bootstrap HEGY tests were little different to those reported here for the case of $e_{4 n+s}$ IID standard normal.

${ }^{6} \mathrm{We}$ also considered the cases of a double volatility shift and trending volatility but found the results to be qualitatively similar for the results reported for Model 3.
} 
length by $\hat{p}$, we also then set $p^{*}=\hat{p}$ in Step 4 of Algorithm 1 for all of the simulation results we report here. To obtain our estimate $\hat{p}$ we propose a seasonal generalisation of the heteroskedasticity-robust re-scaled modified information criterion [MIC] based method of Cavaliere et al. (2015), designed to account for non-stationary volatility in the shocks by using re-scaled data. Our suggested approach consists of applying their re-scaling approach to each of the seasons separately. To that end, first partition the time series into $S$ separate series, one for each of the seasons; that is, define the $N \times 1$ vectors, $\breve{x}_{s, n}:=x_{S n+s}, n=1, \ldots, N$, for each of $s=1-S, \ldots, 0$. Then, for each of these $S$ series, we separately apply the re-scaling approach outlined in equations (6) and (7) of section 3.3 of Cavaliere et al. (2015), which is based on the approach of Beare (2008), to yield the resulting re-scaled series, say $\breve{x}_{s, n}^{r}$. Specifically, for each of $s=1-S, \ldots, 0$, define

$$
\breve{x}_{s, n}^{r}:=\sum_{j=1}^{n} \frac{\left(\breve{x}_{s, n}^{d}-\breve{x}_{s, n-1}^{d}\right)}{\hat{\omega}_{N}(j / N)}, n=2, \ldots, N, \breve{x}_{s, 1}^{r}=0
$$

where $\breve{x}_{s, n}^{d}$ denotes the OLS de-trended version (allowing for a constant and trend) of $\breve{x}_{s, n}$, and

$$
\hat{\omega}_{N}(r):=\left(\frac{\sum_{n=1}^{N} k\left(\frac{n / N-r}{b_{N}}\right)\left(e_{p^{\dagger}, n}\right)^{2}}{\sum_{n=1}^{N} k\left(\frac{n / N-r}{b_{N}}\right)}\right)^{1 / 2},
$$

where $e_{p^{\dagger}, n}$ are residuals from the ADF regression applied to $\breve{x}_{s, n}^{d}$, for each of $s=1-S, \ldots, 0$ in each case with a lag length $p^{\dagger}$ (we follow Cavaliere et al. (2015) and set $p^{\dagger}=0$ ), $k(\cdot)$ is a kernel function and $b_{N}$ a bandwidth satisfying the conditions given in Assumption 4 of Cavaliere et al. (2015). In the results reported in this paper we follow Cavaliere et al. (2015) and use the Gaussian kernel for $k(\cdot)$ and set the bandwidth to be $b_{N}=0.1$. The filtered series are then merged back into a single filtered series; viz., $x_{S n+s}^{r}:=\breve{x}_{s, n}^{r}, s=1-S, \ldots, 0, n=1, \ldots, N$.

The MIC approach of Ng and Perron (2001) originally developed for the non-seasonal ADF test and generalised to the seasonal case by del Barrio Castro et al. (2016) can then be applied to the re-scaled data, $x_{S n+s}^{r}$. That is, the lag length $\hat{p}$ in (9) is chosen such that

$$
\hat{p}:=\underset{0 \leq p \leq p_{\max }}{\operatorname{argmin}} \operatorname{MIC}(p), \quad M I C(p):=\ln \left(\hat{\sigma}_{p, r}^{2}\right)+\frac{C_{T}\left[\tau_{T}(p)+p\right]}{T-p_{\max }} .
$$

In the context of (19), $\hat{\sigma}_{p, r}^{2}:=S S R_{p, r} /\left(T-p_{\max }\right)$, with $S S R_{p, r}$ the sum of squared residuals from applying the HEGY regression in (9) to $\hat{x}_{S n+s}^{r}$, the OLS de-trended analogue of $x_{S n+s}^{r}$ (following the arguments given in Perron and Qu (2007) only OLS de-trending is used in the lag selection element), with $p$ lagged differences. The penalty term $\tau_{T}(p)$ is given by

$$
\begin{aligned}
& \tau_{T}(p):=\left(1 / \hat{\sigma}_{p, r}^{2}\right)\left[\hat{\pi}_{0, r}^{2} \sum_{n} \sum_{s}\left(\hat{x}_{0, S n+s-1}^{r}\right)^{2}+\hat{\pi}_{S / 2, r}^{2} \sum_{n} \sum_{s}\left(\hat{x}_{S / 2, S n+s-1}^{r}\right)^{2}\right. \\
&\left.+\sum_{k=1}^{S^{*}}\left(\hat{\pi}_{\alpha, k, r}^{2} \sum_{n} \sum_{s}\left(\hat{x}_{k, S n+s-1}^{\alpha, r}\right)^{2}+\hat{\pi}_{\beta, k, r}^{2} \sum_{n} \sum_{s}\left(\hat{x}_{k, S n+s-1}^{\beta, r}\right)^{2}\right)\right]
\end{aligned}
$$


using obvious notation for the fitted parameters and HEGY transforms from (9) applied to $\hat{x}_{S n+s}^{r}$. The associated penalty function parameter, $C_{T}$, depends on the specific criterion adopted; in all of the results reported in this paper we use $C_{T}=2$, yielding a re-scaled modified AIC [MAIC] criterion, and using $p_{\max }=\left\lfloor 12(T / 100)^{1 / 4}\right\rfloor$ throughout. We compared the re-scaled MAIC lag selection method outlined above with the MAIC method of del Barrio Castro et al. (2016) (which is calculated as above but using $x_{S n+s}$ in place of $x_{S n+s}^{r}$ ) and, like Cavaliere et al. (2015) for the non-seasonal case, found the former to perform considerably better. These results are available on request.

\subsection{Empirical Size}

\section{Heteroskedasticity but no Serial Correlation}

We first investigate the empirical size properties of the standard HEGY $t_{0}, t_{2}, F_{1}, F_{12}$ and $F_{012}$ tests together with their wild bootstrap counterparts $t_{0}^{*}, t_{2}^{*}, F_{1}^{*}, F_{12}^{*}$ and $F_{012}^{*}$ in the case where the shocks are heteroskedastic but do not contain any weak dependence. To that end, the results reported in Tables 1 and 2 for OLS and local GLS de-trending, respectively, relate to data generated according to (14)-(16) under $H_{0}$ of (4), so that $\alpha(L)=\left(1-L^{4}\right)$, for each of Models 1-4 with $\phi(L)=\theta(L)=1$.

Consider first the results in Tables 1 and 2 for the homoskedastic case of Model 1. Here there are mostly no significant differences between the empirical rejection frequencies [ERFs] of the standard HEGY tests and their wild bootstrap counterparts, particularly so for $N=100$. However, for $N=50$ the OLS de-trended $t_{0}^{*}$ and $t_{2}^{*}$ wild bootstrap tests are seen to avoid the significant under-sizing seen with their standard HEGY counterparts, while the local GLS de-trended $t_{2}^{*}, F_{1}^{*}, F_{12}^{*}$ and $F_{012}^{*}$ tests avoid the over-sizing seen with their standard HEGY counterparts.

Consider next the results For Model 2 where standard PH is present in the errors. Here we observe results for the standard HEGY tests which are qualitatively similar to those reported in Burridge and Taylor (2001a). As discussed in Burridge and Taylor (2001a), the asymptotic sizes of the HEGY $F_{1}$ test (and, hence, also of the HEGY $F_{12}$ and $F_{012}$ tests) increase as the ratio of $\left(\sigma_{0,-3}^{2}+\sigma_{0,-1}^{2}\right)$ to $\left(\sigma_{0,-2}^{2}+\sigma_{0,0}^{2}\right)$ increases, while the asymptotic sizes of the $t_{0}$ and $t_{2}$ tests are unaffected regardless of the value of this ratio. The results in Tables 1 and 2 bear out these asymptotic predictions; in particular, while the standard $t_{0}$ and $t_{2}$ HEGY tests display ERFs close to the nominal level (albeit less so for the local GLS de-trended implementations of these tests), the $F_{1}, F_{12}$ and $F_{012}$ tests all display ERFs well in excess of the nominal level, with these size distortions increasing as the degree of PH increases. These size distortions are also seen not to ameliorate as the sample size is increased, again as predicted by the asymptotic theory. In contrast the bootstrap HEGY tests display ERFs close to the nominal level throughout.

Consider next Model 3, a single volatility shift. Here, the potential for significant size distortions in the standard HEGY tests is clearly seen in the results. Consistent with the findings of Cavaliere and Taylor (2008a) for non-seasonal unit root tests, the most pronounced examples of over-sizing are seen for the case of early negative $(\delta=3$ and $\tau=0.2)$ breaks for the OLS de-trended HEGY tests, and late positive $(\delta=1 / 3$ and $\tau=0.8)$ breaks for the local GLS de-trended tests. In the former case 
the observed ERFs are seen to exceed $50 \%$ for some of the tests. Additionally, it is seen that the OLS de-trended tests can be very conservative for early positive $(\delta=1 / 3$ and $\tau=0.2)$ breaks. In contrast the wild bootstrap HEGY tests, particularly those based on local GLS de-trending, display very good size control throughout Tables 1 and 2 under Model 3. Some over-sizing remains for $N=50$ in the OLS de-trended HEGY tests under early negative breaks, but this is largely eliminated by $N=100$.

Consider finally the results in Tables 1 and 2 relating to Model 4. The results reported here are largely consistent with those given for the pure PH and single volatility shift cases considered above in Models 2 and 3, respectively, again bearing in mind the discussion from Burridge and Taylor (2001a) regarding the dependence of the asymptotic size of the HEGY tests on the ratio of the periodic variances. So we again see that early negative periodic volatility shifts have the greatest impact on the OLS de-trended HEGY tests while late positive shifts have the greatest impact on the local GLS de-trended tests. Under Case 3 the volatility shift only affects the second and fourth quarters and it appears that this serves to ameliorate the size distortions relative to Cases 1 and 2 where the volatility break affects all of the seasons. Notice also that the size distortions seen for the $t_{0}$ and $t_{2}$ tests under Case 4 are relatively small, as would be expected given that here the annual average of the variances of the innovations is constant across the sample; cf. Remark 8. The wild bootstrap HEGY tests again do a very good job of controlling size under Model 4. As in the single volatility shift case, although some size distortions are still seen for $N=50$ these are considerably lower than the size distortions seen in the corresponding standard HEGY tests and are ameliorated for $N=100$. Once again the local GLS de-trended wild bootstrap HEGY tests would appear to deliver superior finite sample size control to their OLS de-trended counterparts. It is worth noting that some of the cases considered here display enormous PH, whose form undergoes a break, that would never be seen in practice. Even for these pathological cases the wild bootstrap HEGY tests deliver decent size control in finite samples.

\section{Heteroskedasticity and Serial Correlation}

We next investigate the empirical size properties of the standard and wild bootstrap HEGY tests in the case where the shocks can display both heteroskedastic and parametric weak dependence. To that end, the results reported in Tables 3 and 4, for OLS and local GLS de-trending, respectively, relate to data generated according to (14)-(16) with $\alpha(L)=\left(1-L^{4}\right)$ and for both the case of firstorder seasonal AR shocks, $\phi(L)=\left(1-\Phi L^{4}\right), \theta(L)=1$, and where the shocks follow an $M A(2)$ process, viz., $\theta(L)=\left(1-\Theta L^{2}\right), \phi(L)=1$, in each case initialised at zero. Results are reported for $\Phi=\{-0.8,-0.5,0.5,0.8\}$ in the seasonal AR case, and for $\Theta=\{-0.5,0.5\}$ in the MA case. In terms of heteroskedasticity, we report results for a selection of the Models considered above, namely: Model 1; Model 2, Case 2; Model 3 for (i) $\delta=3, \tau=0.8$, and (ii) $\delta=3, \tau=0.2$; Model 4, Case 3 for $\delta=3$, $\tau=0.2$.

The results in Tables 3 and 4 provide a similar message to those seen in Tables 1 and 2. Significant finite sample size distortions are again seen in the standard HEGY tests for many of the heteroskedastic models considered with the wild bootstrap implementations of the HEGY tests again delivering good finite sample size control. In those cases where heteroskedasticity has a pronounced impact on the 
ERFs of the standard HEGY tests, the impact of weak dependence on the tests is seen to be rather small in comparison in most cases. In the homoskedastic case weak dependence appears to have relatively little impact on the OLS de-trended HEGY tests but can lead to some finite sample oversizing in the local GLS de-trended HEGY tests, most notably in the $M A(2)$ case with $\Theta=-0.5$. Even here the wild corresponding wild bootstrap tests are seen to deliver some improvements in finite sample size relative to the standard HEGY tests.

\subsection{Empirical Power}

We now turn to a consideration of the relative finite sample power properties of the standard and wild bootstrap HEGY tests. In order to take account of the differing empirical size properties of the standard HEGY tests and their wild bootstrap analogues that we have seen can occur under heteroskedasticity, we report size-adjusted powers for the standard HEGY tests. The size-adjustment is done such that the resulting ERFs of a given HEGY test and its wild bootstrap counterpart coincide under the null hypothesis, thereby enabling a meaningful power comparison. Tables 5 and 6 for OLS and local GLS de-trended data, respectively, report the ERFs for the bootstrap HEGY tests and the size-adjusted powers of the standard HEGY tests for data generated by (14)-(16) for each of Models 1-4 under the near-seasonally integrated alternative $\alpha(L)=\left(1-(1+c / N) L^{4}\right)$ whereby $x_{4 n+s}$ is nearintegrated at each of the zero, Nyquist and harmonic seasonal frequencies; see Rodrigues and Taylor (2004, pp.648-649) for further details. Results are reported for $c \in\{-3.75,-7,-13.5\}$. Notice that, under homoskedasticity, the $t_{0}$ test when based on local GLS de-trending has power that will approach $50 \%$ for $c=-13.5$ as $N \rightarrow \infty$, while the $t_{2}$ test based on GLS de-trended data will have power which approaches $50 \%$ for $c=-7$; see, for example, Rodrigues and Taylor (2007). The reported results pertain to the serially uncorrelated case, $\phi(L)=\theta(L)=1$.

The main conclusions that can be drawn from the results in Tables 5 and 6 can be easily summarised. First, and as predicted by the asymptotic distribution theory (see Remark 15), the finite sample power of the wild bootstrap HEGY tests is very similar to the size-adjusted power of the corresponding standard HEGY tests throughout. This coincidence holds both when OLS de-trending is used and when local GLS de-trending is used. In the homoskedastic case (Model 1) in particular this observation implies that there is no significant loss in finite sample power incurred from using the wild bootstrap, yet at the same time the wild bootstrap was seen to largely correct the size distortions that can be incurred by the standard HEGY tests under heteroskedasticity. Second, the power of both the standard and wild bootstrap HEGY tests depends on the pattern of heteroskedasticity present in the shocks. In some cases power can be rendered very low indeed, most notably in the single volatility shift case with $\delta=3$ and $\tau=0.2$ when OLS de-trending is employed. This was, of course, exactly the case where the most significant size distortions were seen in the standard HEGY tests under the null; cf. Tables 1 and 2. Interestingly, the finite sample powers of the local GLS de-trended tests appear overall to be considerably less affected by heteroskedasticity than their OLS de-trended counterparts, although counterexamples to this general rule can be seen in the results. 


\section{Conclusions}

In this paper we have investigated the behaviour of the so-called HEGY regression-based seasonal unit root tests of Hylleberg et al. (1990) when the driving shocks can display both periodic non-stationary volatility and conditional heteroskedasticity. The general set-up we have considered for the volatility process of the shocks includes the familiar examples of periodic heteroskedasticity, non-stationary volatility and GARCH as special cases. We have shown that such patterns of non-constant volatility lead, in general, to the presence of nuisance parameters in the limiting null distributions of the HEGY tests. Monte Carlo simulation methods presented for a number of non-constant volatility processes have been used to demonstrate that this can lead to serious size distortions in the HEGY tests when based on conventional critical values designed for the homoskedastic case. We have shown that this inference problem can be solved, at least asymptotically, by using bootstrap implementations of the HEGY tests, based around wild bootstrap re-sampling schemes. Simulations have shown that the resulting wild bootstrap HEGY tests control size well in finite samples and have power close to that of the size-adjusted power of the standard HEGY tests.

\section{References}

Beare, B.K. (2008). Unit root testing with unstable volatility. Unpublished Manuscript.

Boswijk, H.P., Cavaliere, G., Rahbek, A. and Taylor, A.M.R. (2016). Inference on co-integration parameters in heteroskedastic vector autoregressions. Journal of Econometrics, 192, 64-85.

Burridge, P. and Taylor, A.M.R. (2001a). On regression-based tests for seasonal unit roots in the presence of periodic heteroscedasticity. Journal of Econometrics, 104, 91-117.

Burridge, P. and Taylor, A.M.R. (2001b). On the properties of regression-based tests for seasonal unit roots in the presence of higher-order serial correlation. Journal of Business \& Economic Statistics, $19,374-379$.

Burridge, P. and Taylor, A.M.R. (2004). Bootstrapping the HEGY seasonal unit root tests. Journal of Econometrics, 123, 67-87.

Cavaliere, G. (2004). Unit root tests under time-varying variances. Econometric Reviews, 23, 259-292.

Cavaliere, G., Phillips, P.C.B., Smeekes, S., and Taylor, A.M.R. (2015). Lag Length Selection for Unit Root Tests in the Presence of Nonstationary Volatility. Econometric Reviews, 34, 512-536.

Cavaliere, G., Rahbek, A., and Taylor, A.M.R. (2010). Testing for co-integration in vector autoregressions with non-stationary volatility. Journal of Econometrics, 158, 7-24.

Cavaliere, G. and Taylor, A.M.R. (2007). Testing for unit roots in time series models with nonstationary volatility. Journal of Econometrics, 140, 919-947. 
Cavaliere, G. and Taylor, A.M.R. (2008a). Bootstrap unit root tests for time series with nonstationary volatility. Econometric Theory, 24, 43-71.

Cavaliere, G. and Taylor, A.M.R. (2008b). Time-transformed unit root tests for models with nonstationary volatility. Journal of Time Series Analysis, 29, 300-330.

Cavaliere, G. and Taylor, A.M.R. (2009). Bootstrap M unit root tests. Econometric Reviews, 28, 393-421.

Davidson, J. (1994). Stochastic Limit Theory. Oxford University Press, Oxford.

del Barrio Castro, T., Osborn, D.R., and Taylor, A.M.R. (2012). On augmented HEGY tests for seasonal unit roots. Econometric Theory, 28, 1121-1143.

del Barrio Castro, T., Osborn, D.R., and Taylor, A.M.R. (2016). The Performance of Lag Selection and Detrending Methods for HEGY Seasonal Unit Root Tests. Econometrics Reviews 35, 122-168

Ghysels, E., Lee, H.S., and Noh, J. (1994). Testing for unit roots in seasonal time series: some theoretical extensions and a Monte Carlo investigation. Journal of Econometrics, 62, 415-442.

Giné, E. and Zinn, J. (1990). Bootstrapping general empirical measures. Annals of Probability, 18, 851-869.

Hansen, B.E. (2000). Testing for structural change in conditional models. Journal of Econometrics, 97, 93-115.

Hylleberg, S., Engle, R.F., Granger, C.W.J., and Yoo, B.S. (1990). Seasonal integration and cointegration. Journal of Econometrics, 44, 215-238.

Kim, C.-J. and Nelson, C.R. (1999). Has the US economy become more stable? A Bayesian approach based on a Markov-switching model of the business cycle. Review of Economics and Statistics, 81, 608-616.

McConnell, M.M. and Perez-Quiros, G. (2000). Output fluctuations in the United States: What has changed since the early 1980s? American Economic Review, 90, 1464-1476.

Ng, S. and Perron, P. (2001). Lag length selection and the construction of unit root tests with good size and power, Econometrica 69, 1519-1554.

Perron, P. and Qu, Z. (2007). A simple modification to improve the finite sample properties of $\mathrm{Ng}$ and Perron's unit root tests, Economics Letters 94, 12-19.

Rodrigues, P.M.M. and Taylor, A.M.R. (2004). Asymptotic distributions for regression-based seasonal unit root test statistics in a near-integrated model. Econometric Theory, 20, 645-670.

Rodrigues, P.M.M. and Taylor, A.M.R. (2007). Efficient tests of the seasonal unit root hypothesis. Journal of Econometrics, 141, 548-573. 
Sensier, M. and van Dijk, D. (2004). Testing for volatility changes in U.S. macroeconomic time series. Review of Economics and Statistics, 86, 833-839.

Shao, X. (2011). A bootstrap-assisted spectral test of white noise under unknown dependence. Journal of Econometrics, 162, 213-224.

Shephard, N. (2005). Stochastic Volatility: Selected Readings. Oxford University Press, Oxford.

Smith, R.J., Taylor, A.M.R., and del Barrio Castro, T. (2009). Regression-based seasonal unit root tests. Econometric Theory, 25, 527-560.

Smith, R.J. and Taylor, A.M.R. (1998). Additional critical values and asymptotic representations for seasonal unit root tests. Journal of Econometrics, 85, 269-288.

Wu, C.F.J. (1986), Jackknife, bootstrap, and other resampling methods, Annals of Statistics, 14, 1261-1295.

Zou, N. and Politis, D. (2016). HEGY test under seasonal heterogeneity. Working Paper, Department of Economics, University of California-San Diego. 
Table 1. Empirical sizes of conventional and wild bootstrap HEGY tests using Step 2a of Algorithm 1. OLS de-trending.

\begin{tabular}{|c|c|c|c|c|c|c|c|c|c|c|c|c|c|}
\hline$\Sigma$ & $\delta$ & $\tau$ & $N$ & $t_{0}$ & $t_{2}$ & $F_{1}$ & $F_{12}$ & $F_{012}$ & $t_{0}^{*}$ & $t_{2}^{*}$ & $F_{1}^{*}$ & $F_{12}^{*}$ & $F_{012}^{*}$ \\
\hline \multicolumn{14}{|c|}{ Model 1: Homoskedasticity } \\
\hline & & & 50 & 3.1 & 4.6 & 3.7 & 4.6 & 4.4 & 4.1 & 5.3 & 4.7 & 4.9 & 4.8 \\
\hline & & & 100 & 3.9 & 4.7 & 4.2 & 4.6 & 4.7 & 4.5 & 5.2 & 4.6 & 4.6 & 4.8 \\
\hline \multicolumn{14}{|c|}{ Model 2: Periodic heteroskedasticity } \\
\hline \multirow[t]{2}{*}{ Case 1} & & & 50 & 4.0 & 4.7 & 6.0 & 6.3 & 6.6 & 4.9 & 5.4 & 5.8 & 5.9 & 5.8 \\
\hline & & & 100 & 4.5 & 5.1 & 6.5 & 6.4 & 6.9 & 5.1 & 5.4 & 5.5 & 5.5 & 5.5 \\
\hline Case 2 & & & 50 & 3.7 & 4.4 & 9.1 & 13.2 & 13.0 & 5.1 & 5.8 & 6.2 & 6.4 & 6.1 \\
\hline & & & 100 & 4.2 & 4.9 & 9.4 & 13.3 & 13.0 & 5.0 & 5.3 & 5.7 & 5.8 & 5.4 \\
\hline \multicolumn{14}{|c|}{ Model 3: Single volatility shift } \\
\hline & 0.33 & 0.2 & 50 & 1.9 & 2.1 & 1.4 & 1.4 & 1.4 & 3.6 & 4.5 & 4.1 & 4.3 & 4.0 \\
\hline & & & 100 & 2.6 & 2.3 & 1.6 & 1.5 & 1.3 & 4.2 & 4.8 & 4.3 & 4.4 & 4.0 \\
\hline & & 0.8 & 50 & 5.0 & 5.0 & 6.5 & 6.9 & 6.4 & 4.1 & 4.9 & 5.3 & 5.4 & 4.9 \\
\hline & & & 100 & 6.1 & 5.9 & 7.6 & 7.9 & 7.4 & 4.4 & 4.9 & 5.3 & 5.2 & 5.1 \\
\hline & 3 & 0.2 & 50 & 20.1 & 27.5 & 42.6 & 54.6 & 61.5 & 7.4 & 8.5 & 9.6 & 10.9 & 11.3 \\
\hline & & & 100 & 21.6 & 29.0 & 44.4 & 56.8 & 62.3 & 6.0 & 6.5 & 7.4 & 8.0 & 8.0 \\
\hline & & 0.8 & 50 & 4.7 & 6.3 & 6.6 & 7.7 & 8.4 & 4.2 & 5.4 & 5.3 & 5.2 & 4.9 \\
\hline & & & 100 & 5.6 & 6.4 & 6.8 & 7.7 & 8.6 & 4.6 & 4.6 & 4.8 & 4.6 & 4.7 \\
\hline \multicolumn{14}{|c|}{ Model 4: Single periodic volatility shift } \\
\hline \multirow[t]{8}{*}{ Case 1} & 0.33 & 0.2 & 50 & 2.5 & 2.1 & 1.8 & 1.6 & 1.8 & 4.3 & 4.6 & 4.4 & 4.3 & 4.3 \\
\hline & & & 100 & 2.8 & 2.3 & 2.2 & 1.6 & 1.9 & 4.6 & 4.8 & 4.4 & 4.5 & 4.4 \\
\hline & & 0.8 & 50 & 5.4 & 5.3 & 6.9 & 7.0 & 7.7 & 4.6 & 5.0 & 5.4 & 5.5 & 5.5 \\
\hline & & & 100 & 6.7 & 6.1 & 7.8 & 7.8 & 8.3 & 5.0 & 5.0 & 5.3 & 5.2 & 5.0 \\
\hline & 3 & 0.2 & 50 & 19.8 & 27.5 & 40.0 & 51.5 & 56.9 & 7.6 & 8.8 & 9.4 & 10.8 & 11.3 \\
\hline & & & 100 & 21.5 & 28.6 & 40.7 & 53.3 & 57.8 & 6.7 & 7.1 & 7.4 & 8.0 & 8.3 \\
\hline & & 0.8 & 50 & 4.9 & 6.5 & 8.3 & 9.2 & 10.4 & 4.6 & 5.5 & 5.8 & 5.8 & 5.5 \\
\hline & & & 100 & 5.7 & 6.8 & 8.6 & 9.2 & 10.7 & 4.7 & 5.0 & 5.0 & 5.2 & 5.2 \\
\hline \multirow[t]{8}{*}{ Case 2} & 0.33 & 0.2 & 50 & 2.5 & 2.3 & 4.6 & 6.5 & 6.9 & 5.2 & 4.9 & 5.2 & 5.0 & 5.1 \\
\hline & & & 100 & 2.8 & 2.4 & 4.4 & 6.5 & 6.8 & 4.9 & 4.8 & 4.9 & 4.9 & 4.9 \\
\hline & & 0.8 & 50 & 5.8 & 6.3 & 10.8 & 14.2 & 14.6 & 6.0 & 6.3 & 6.6 & 6.7 & 6.7 \\
\hline & & & 100 & 6.2 & 6.0 & 11.0 & 14.6 & 15.1 & 5.4 & 5.5 & 6.1 & 6.1 & 5.7 \\
\hline & 3 & 0.2 & 50 & 18.3 & 27.1 & 38.1 & 44.8 & 47.4 & 9.3 & 11.6 & 12.4 & 12.5 & 12.8 \\
\hline & & & 100 & 20.7 & 27.9 & 38.3 & 45.0 & 47.3 & 7.2 & 7.8 & 8.3 & 8.4 & 8.4 \\
\hline & & 0.8 & 50 & 5.0 & 6.3 & 12.0 & 16.5 & 16.6 & 5.7 & 6.1 & 6.6 & 6.7 & 6.5 \\
\hline & & & 100 & 6.0 & 7.6 & 12.7 & 17.5 & 17.5 & 5.5 & 6.1 & 6.2 & 6.4 & 6.0 \\
\hline \multirow[t]{8}{*}{ Case 3} & 0.33 & 0.2 & 50 & 2.4 & 2.1 & 2.2 & 1.8 & 2.2 & 4.4 & 4.9 & 4.6 & 4.9 & 4.6 \\
\hline & & & 100 & 2.9 & 2.2 & 2.5 & 2.2 & 2.3 & 4.3 & 4.4 & 4.6 & 4.5 & 4.3 \\
\hline & & 0.8 & 50 & 3.7 & 4.4 & 4.2 & 4.4 & 4.7 & 4.1 & 5.2 & 5.1 & 5.3 & 4.9 \\
\hline & & & 100 & 4.6 & 4.9 & 4.7 & 4.8 & 5.0 & 4.8 & 5.0 & 4.9 & 4.8 & 4.8 \\
\hline & 3 & 0.2 & 50 & 5.7 & 7.4 & 10.5 & 11.5 & 12.4 & 5.4 & 5.6 & 6.1 & 5.9 & 5.6 \\
\hline & & & 100 & 5.8 & 8.2 & 11.3 & 12.4 & 12.5 & 5.0 & 5.2 & 5.4 & 5.4 & 5.3 \\
\hline & & 0.8 & 50 & 3.7 & 5.0 & 5.0 & 5.4 & 5.8 & 4.3 & 5.4 & 5.1 & 4.9 & 4.6 \\
\hline & & & 100 & 4.4 & 5.3 & 5.3 & 5.7 & 5.9 & 4.7 & 4.7 & 4.9 & 4.5 & 4.4 \\
\hline \multirow[t]{4}{*}{ Case 4} & & 0.2 & 50 & 3.4 & 4.5 & 6.0 & 6.1 & 6.8 & 4.3 & 5.1 & 5.4 & 5.2 & 4.7 \\
\hline & & & 100 & 4.0 & 4.7 & 6.3 & 6.1 & 6.6 & 4.5 & 5.3 & 5.4 & 5.2 & 4.8 \\
\hline & & 0.8 & 50 & 3.8 & 4.2 & 5.3 & 5.4 & 6.1 & 4.2 & 5.1 & 5.4 & 5.2 & 4.9 \\
\hline & & & 100 & 4.0 & 4.6 & 5.7 & 5.8 & 6.2 & 4.6 & 5.5 & 5.5 & 5.5 & 5.3 \\
\hline
\end{tabular}


Table 2. Empirical sizes of conventional and wild bootstrap HEGY tests using Step 2a of Algorithm 1. Local GLS de-trending.

\begin{tabular}{|c|c|c|c|c|c|c|c|c|c|c|c|c|c|}
\hline$\Sigma$ & $\delta$ & $\tau$ & $N$ & $t_{0}$ & $t_{2}$ & $F_{1}$ & $F_{12}$ & $F_{012}$ & $t_{0}^{*}$ & $t_{2}^{*}$ & $F_{1}^{*}$ & $F_{12}^{*}$ & $F_{012}^{*}$ \\
\hline \multicolumn{14}{|c|}{ Model 1: Homoskedasticity } \\
\hline & & & 50 & 5.9 & 8.7 & 6.6 & 8.1 & 8.7 & 4.1 & 4.7 & 4.9 & 5.1 & 4.6 \\
\hline & & & 100 & 5.2 & 7.2 & 5.8 & 5.8 & 6.7 & 4.3 & 4.9 & 4.7 & 4.6 & 4.8 \\
\hline \multicolumn{14}{|c|}{ Model 2: Periodic heteroskedasticity } \\
\hline \multirow[t]{2}{*}{ Case 1} & & & 50 & 6.5 & 9.6 & 6.5 & 7.5 & 9.6 & 4.7 & 5.5 & 4.9 & 5.2 & 4.8 \\
\hline & & & 100 & 6.1 & 7.1 & 5.7 & 6.3 & 7.9 & 5.0 & 5.0 & 4.9 & 5.1 & 5.2 \\
\hline Case 2 & & & 50 & 6.1 & 9.1 & 10.1 & 13.3 & 13.6 & 5.2 & 5.1 & 5.6 & 5.7 & 5.8 \\
\hline & & & 100 & 5.2 & 7.5 & 9.7 & 12.5 & 12.2 & 4.6 & 5.2 & 5.5 & 5.5 & 5.4 \\
\hline \multicolumn{14}{|c|}{ Model 3: Single volatility shift } \\
\hline & 0.33 & 0.2 & 50 & 6.7 & 10.1 & 9.3 & 10.9 & 11.4 & 4.1 & 4.6 & 4.9 & 5.1 & 4.5 \\
\hline & & & 100 & 6.3 & 8.7 & 9.0 & 9.5 & 9.9 & 4.2 & 4.9 & 4.9 & 4.8 & 4.7 \\
\hline & & 0.8 & 50 & 10.2 & 14.6 & 19.7 & 24.5 & 24.2 & 4.3 & 5.2 & 6.0 & 6.0 & 5.8 \\
\hline & & & 100 & 10.7 & 12.6 & 19.7 & 23.7 & 23.3 & 4.6 & 4.9 & 5.6 & 5.6 & 5.6 \\
\hline & 3 & 0.2 & 50 & 11.6 & 13.5 & 6.6 & 9.9 & 14.0 & 5.4 & 5.8 & 5.6 & 6.1 & 5.8 \\
\hline & & & 100 & 9.2 & 10.2 & 5.7 & 7.4 & 9.6 & 5.0 & 5.2 & 5.5 & 5.6 & 5.1 \\
\hline & & 0.8 & 50 & 6.7 & 9.3 & 5.6 & 6.9 & 9.0 & 4.1 & 4.7 & 4.8 & 4.6 & 4.4 \\
\hline & & & 100 & 6.1 & 7.3 & 4.7 & 5.2 & 6.2 & 4.3 & 4.6 & 4.6 & 4.5 & 4.5 \\
\hline \multicolumn{14}{|c|}{ Model 4: Single periodic volatility shift } \\
\hline Case 1 & 0.33 & 0.2 & 50 & 7.3 & 10.5 & 9.3 & 10.6 & 12.3 & 4.6 & 4.9 & 5.2 & 5.3 & 4.9 \\
\hline & & & 100 & 6.8 & 9.0 & 8.4 & 9.5 & 10.7 & 4.7 & 4.8 & 5.2 & 4.9 & 4.9 \\
\hline & & 0.8 & 50 & 10.8 & 14.8 & 17.7 & 21.6 & 23.5 & 5.0 & 5.2 & 6.0 & 6.0 & 5.7 \\
\hline & & & 100 & 10.8 & 12.6 & 17.2 & 21.2 & 22.5 & 5.0 & 5.4 & 5.7 & 5.9 & 5.5 \\
\hline & 3 & 0.2 & 50 & 11.4 & 12.9 & 7.1 & 10.0 & 15.2 & 5.7 & 5.6 & 5.7 & 6.1 & 6.5 \\
\hline & & & 100 & 9.4 & 9.8 & 6.0 & 7.3 & 10.7 & 5.0 & 5.0 & 5.3 & 5.2 & 5.0 \\
\hline & & 0.8 & 50 & 7.2 & 9.6 & 5.9 & 6.9 & 10.0 & 4.5 & 4.7 & 5.2 & 4.7 & 4.7 \\
\hline & & & 100 & 6.7 & 7.3 & 4.7 & 5.3 & 7.4 & 5.1 & 4.7 & 4.8 & 4.8 & 5.0 \\
\hline \multirow[t]{8}{*}{ Case 2} & 0.33 & 0.2 & 50 & 7.0 & 10.5 & 12.8 & 16.1 & 16.1 & 5.2 & 5.3 & 5.9 & 5.7 & 5.8 \\
\hline & & & 100 & 6.8 & 8.6 & 11.9 & 14.9 & 14.8 & 4.8 & 4.7 & 5.0 & 5.1 & 5.0 \\
\hline & & 0.8 & 50 & 11.2 & 15.4 & 20.2 & 24.3 & 24.4 & 6.4 & 6.3 & 7.8 & 7.7 & 7.3 \\
\hline & & & 100 & 10.5 & 12.7 & 20.1 & 23.7 & 23.2 & 5.5 & 5.3 & 6.8 & 6.6 & 6.4 \\
\hline & 3 & 0.2 & 50 & 11.2 & 13.3 & 12.0 & 15.7 & 19.0 & 6.9 & 6.9 & 7.1 & 7.1 & 7.4 \\
\hline & & & 100 & 8.8 & 9.8 & 10.1 & 13.7 & 15.2 & 5.6 & 5.5 & 5.6 & 5.6 & 5.7 \\
\hline & & 0.8 & 50 & 7.0 & 10.2 & 9.9 & 13.7 & 14.5 & 5.4 & 5.5 & 6.0 & 5.8 & 5.6 \\
\hline & & & 100 & 6.6 & 8.1 & 9.1 & 12.0 & 12.5 & 5.3 & 5.4 & 5.3 & 5.6 & 5.3 \\
\hline \multirow[t]{8}{*}{ Case 3} & 0.33 & 0.2 & 50 & 7.0 & 10.0 & 7.7 & 9.8 & 11.7 & 4.5 & 5.3 & 5.1 & 5.0 & 4.9 \\
\hline & & & 100 & 6.6 & 8.5 & 7.5 & 8.1 & 9.5 & 4.6 & 4.7 & 5.1 & 4.9 & 4.9 \\
\hline & & 0.8 & 50 & 8.0 & 11.8 & 12.5 & 15.3 & 16.5 & 4.2 & 5.2 & 5.8 & 5.7 & 5.3 \\
\hline & & & 100 & 7.9 & 9.2 & 12.5 & 14.2 & 14.5 & 4.9 & 4.7 & 5.2 & 5.6 & 5.2 \\
\hline & 3 & 0.2 & 50 & 6.4 & 8.7 & 4.9 & 6.3 & 8.4 & 4.5 & 4.3 & 4.8 & 4.9 & 4.6 \\
\hline & & & 100 & 5.5 & 7.1 & 4.6 & 5.1 & 6.2 & 4.7 & 5.0 & 4.9 & 4.7 & 4.8 \\
\hline & & 0.8 & 50 & 6.2 & 8.8 & 5.5 & 6.5 & 8.2 & 4.3 & 4.7 & 4.3 & 4.3 & 4.4 \\
\hline & & & 100 & 5.5 & 6.9 & 5.1 & 5.3 & 6.2 & 4.3 & 4.6 & 4.8 & 4.8 & 4.9 \\
\hline \multirow[t]{4}{*}{ Case 4} & & 0.2 & 50 & 5.8 & 8.8 & 6.1 & 7.3 & 9.0 & 4.2 & 4.6 & 5.0 & 4.8 & 4.4 \\
\hline & & & 100 & 5.5 & 6.8 & 5.3 & 5.7 & 6.8 & 4.2 & 5.1 & 5.1 & 5.1 & 4.9 \\
\hline & & 0.8 & 50 & 6.4 & 8.5 & 6.1 & 7.1 & 9.5 & 4.0 & 4.7 & 4.7 & 4.6 & 4.5 \\
\hline & & & 100 & 5.6 & 7.7 & 5.7 & 5.9 & 7.0 & 4.7 & 5.1 & 4.8 & 4.7 & 4.6 \\
\hline
\end{tabular}


Table 3. Empirical size of conventional and wild bootstrap HEGY tests using Step 2a of Algorithm 1. OLS de-trending. Weakly dependent shocks.

\begin{tabular}{|c|c|c|c|c|c|c|c|c|c|c|c|c|}
\hline & $\Phi / \Theta$ & $N$ & $t_{0}$ & $t_{2}$ & $F_{1}$ & $F_{12}$ & $F_{012}$ & $t_{0}^{*}$ & $t_{2}^{*}$ & $F_{1}^{*}$ & $F_{12}^{*}$ & $F_{012}^{*}$ \\
\hline \multicolumn{13}{|c|}{ Homoskedasticity } \\
\hline \multirow[t]{8}{*}{$\operatorname{AR}(4)$} & -0.8 & 50 & 1.7 & 3.5 & 3.1 & 3.5 & 3.4 & 3.5 & 4.6 & 4.9 & 4.7 & 4.2 \\
\hline & & 100 & 3.1 & 4.4 & 3.9 & 4.2 & 4.0 & 4.1 & 4.7 & 4.9 & 4.8 & 4.8 \\
\hline & -0.5 & 50 & 2.5 & 3.7 & 3.5 & 3.8 & 3.5 & 3.4 & 4.8 & 4.7 & 4.8 & 4.5 \\
\hline & & 100 & 3.5 & 4.1 & 4.0 & 4.3 & 3.9 & 4.3 & 5.0 & 4.9 & 4.9 & 4.9 \\
\hline & 0.5 & 50 & 1.9 & 3.6 & 3.7 & 3.5 & 2.7 & 2.4 & 4.2 & 4.7 & 4.4 & 3.4 \\
\hline & & 100 & 3.6 & 4.4 & 4.3 & 4.5 & 4.3 & 4.7 & 5.0 & 4.8 & 4.5 & 4.5 \\
\hline & 0.8 & 50 & 3.5 & 4.1 & 3.5 & 3.5 & 3.8 & 4.7 & 5.2 & 4.4 & 4.3 & 4.5 \\
\hline & & 100 & 3.7 & 4.6 & 3.7 & 4.0 & 4.0 & 4.5 & 4.9 & 4.2 & 4.4 & 4.7 \\
\hline \multirow[t]{4}{*}{$\operatorname{MA}(2)$} & -0.5 & 50 & 6.7 & 7.6 & 3.2 & 5.4 & 8.3 & 9.4 & 8.9 & 5.0 & 7.0 & 9.8 \\
\hline & & 100 & 6.5 & 6.7 & 3.7 & 5.4 & 6.5 & 7.8 & 7.4 & 4.5 & 6.0 & 7.6 \\
\hline & 0.5 & 50 & 2.8 & 3.7 & 6.2 & 6.2 & 6.0 & 3.6 & 4.7 & 8.3 & 7.3 & 6.7 \\
\hline & & 100 & 2.6 & 4.0 & 5.9 & 5.2 & 4.5 & 3.5 & 4.7 & 6.5 & 6.1 & 5.4 \\
\hline \multicolumn{13}{|c|}{ Periodic heteroskedasticity, Case 2} \\
\hline \multirow[t]{8}{*}{$\operatorname{AR}(4)$} & -0.8 & 50 & 2.9 & 4.1 & 8.1 & 11.6 & 11.4 & 4.6 & 5.6 & 6.0 & 6.1 & 5.9 \\
\hline & & 100 & 3.9 & 4.5 & 9.0 & 12.8 & 12.6 & 4.4 & 5.4 & 5.3 & 5.4 & 5.2 \\
\hline & -0.5 & 50 & 3.3 & 4.4 & 8.8 & 12.5 & 12.5 & 5.5 & 5.7 & 6.1 & 6.1 & 6.1 \\
\hline & & 100 & 4.2 & 4.9 & 9.3 & 12.9 & 13.1 & 4.9 & 5.2 & 5.6 & 5.5 & 5.6 \\
\hline & 0.5 & 50 & 3.0 & 3.9 & 7.8 & 11.1 & 10.8 & 4.0 & 5.0 & 5.4 & 5.5 & 5.1 \\
\hline & & 100 & 3.9 & 4.7 & 9.1 & 13.0 & 13.0 & 5.5 & 5.3 & 5.6 & 5.6 & 5.5 \\
\hline & 0.8 & 50 & 3.8 & 4.5 & 8.7 & 12.6 & 12.2 & 5.4 & 6.1 & 6.4 & 6.5 & 6.3 \\
\hline & & 100 & 4.2 & 4.9 & 9.0 & 12.9 & 12.6 & 5.2 & 5.7 & 5.7 & 5.8 & 5.6 \\
\hline \multirow[t]{4}{*}{$\mathrm{MA}(2)$} & -0.5 & 50 & 4.6 & 4.9 & 7.1 & 11.8 & 12.2 & 7.2 & 7.0 & 5.7 & 6.4 & 6.3 \\
\hline & & 100 & 4.3 & 5.3 & 8.3 & 12.8 & 13.2 & 6.2 & 6.0 & 5.3 & 5.5 & 5.5 \\
\hline & 0.5 & 50 & 2.5 & 3.6 & 10.2 & 13.3 & 12.9 & 4.3 & 5.8 & 8.4 & 7.6 & 7.0 \\
\hline & & 100 & 3.0 & 3.7 & 10.0 & 13.1 & 12.5 & 4.8 & 4.9 & 6.3 & 5.9 & 5.5 \\
\hline \multicolumn{13}{|c|}{ Single volatility shift, $\delta=3, \tau=0.8$} \\
\hline \multirow[t]{8}{*}{$\operatorname{AR}(4)$} & -0.8 & 50 & 2.6 & 5.2 & 5.2 & 6.3 & 6.2 & 3.4 & 4.8 & 5.3 & 4.8 & 4.8 \\
\hline & & 100 & 4.1 & 6.0 & 6.2 & 7.3 & 7.2 & 4.0 & 5.1 & 4.9 & 4.8 & 4.5 \\
\hline & -0.5 & 50 & 3.3 & 5.4 & 5.5 & 6.5 & 6.4 & 3.8 & 5.1 & 5.2 & 5.3 & 5.0 \\
\hline & & 100 & 4.7 & 6.4 & 6.4 & 7.5 & 7.6 & 4.1 & 5.3 & 4.9 & 5.3 & 4.7 \\
\hline & 0.5 & 50 & 2.8 & 4.8 & 5.0 & 5.7 & 5.6 & 3.4 & 4.7 & 4.6 & 4.5 & 3.9 \\
\hline & & 100 & 4.8 & 5.9 & 6.4 & 7.2 & 7.7 & 4.5 & 4.7 & 4.5 & 4.7 & 4.5 \\
\hline & 0.8 & 50 & 4.2 & 5.1 & 4.6 & 5.0 & 5.2 & 4.4 & 4.9 & 4.2 & 4.2 & 4.0 \\
\hline & & 100 & 4.8 & 5.7 & 5.3 & 6.2 & 6.5 & 4.9 & 4.5 & 4.6 & 4.2 & 4.0 \\
\hline \multirow[t]{4}{*}{$\mathrm{MA}(2)$} & -0.5 & 50 & 8.1 & 9.3 & 5.3 & 8.5 & 12.4 & 9.1 & 9.0 & 4.7 & 6.8 & 9.3 \\
\hline & & 100 & 7.3 & 8.4 & 5.7 & 8.4 & 11.0 & 8.0 & 7.1 & 4.6 & 5.7 & 7.2 \\
\hline & 0.5 & 50 & 3.3 & 5.3 & 8.3 & 8.7 & 8.8 & 4.2 & 5.1 & 8.1 & 7.5 & 6.8 \\
\hline & & 100 & 3.7 & 5.5 & 8.2 & 8.7 & 8.2 & 3.8 & 4.5 & 6.3 & 6.0 & 5.2 \\
\hline
\end{tabular}


Table 3. Continued.

\begin{tabular}{|c|c|c|c|c|c|c|c|c|c|c|c|c|}
\hline & $\Phi / \Theta$ & $N$ & $t_{0}$ & $t_{2}$ & $F_{1}$ & $F_{12}$ & $F_{012}$ & $t_{0}^{*}$ & $t_{2}^{*}$ & $F_{1}^{*}$ & $F_{12}^{*}$ & $F_{012}^{*}$ \\
\hline \multicolumn{13}{|c|}{ Single volatility shift, $\delta=3, \tau=0.2$} \\
\hline \multirow[t]{8}{*}{$\operatorname{AR}(4)$} & -0.8 & 50 & 13.6 & 24.4 & 37.4 & 48.3 & 52.1 & 6.4 & 9.4 & 11.8 & 13.3 & 13.3 \\
\hline & & 100 & 18.6 & 26.7 & 41.8 & 53.0 & 57.9 & 6.0 & 6.8 & 8.4 & 9.2 & 8.8 \\
\hline & -0.5 & 50 & 15.8 & 25.2 & 39.1 & 50.6 & 55.0 & 7.5 & 9.5 & 12.4 & 13.6 & 13.9 \\
\hline & & 100 & 20.7 & 27.7 & 41.9 & 53.2 & 59.7 & 6.3 & 7.0 & 8.2 & 8.9 & 8.5 \\
\hline & 0.5 & 50 & 13.3 & 23.5 & 35.6 & 45.3 & 47.3 & 6.8 & 8.0 & 9.6 & 9.8 & 9.6 \\
\hline & & 100 & 17.8 & 26.8 & 40.4 & 51.6 & 55.8 & 6.3 & 6.6 & 6.9 & 6.9 & 7.1 \\
\hline & 0.8 & 50 & 12.4 & 20.1 & 27.9 & 35.3 & 36.2 & 5.9 & 7.1 & 5.7 & 6.1 & 5.9 \\
\hline & & 100 & 15.5 & 24.3 & 35.0 & 44.2 & 47.5 & 5.2 & 5.3 & 4.8 & 4.8 & 4.7 \\
\hline \multirow[t]{4}{*}{$\operatorname{MA}(2)$} & -0.5 & 50 & 22.7 & 31.1 & 35.2 & 50.7 & 59.5 & 11.8 & 11.6 & 10.4 & 13.3 & 16.2 \\
\hline & & 100 & 25.0 & 31.6 & 40.0 & 54.0 & 62.6 & 8.7 & 7.4 & 8.2 & 9.6 & 10.7 \\
\hline & 0.5 & 50 & 14.1 & 24.0 & 45.4 & 54.9 & 57.2 & 6.9 & 8.5 & 13.3 & 13.8 & 13.8 \\
\hline & & 100 & 17.7 & 26.5 & 45.9 & 55.8 & 59.3 & 5.5 & 6.9 & 8.3 & 8.6 & 8.1 \\
\hline \multicolumn{13}{|c|}{ Single periodic volatility shift, $\delta=3, \tau=0.2$, Case 3} \\
\hline \multirow[t]{8}{*}{$\operatorname{AR}(4)$} & -0.8 & 50 & 3.2 & 6.4 & 8.4 & 9.2 & 9.2 & 4.0 & 5.8 & 6.4 & 6.5 & 5.9 \\
\hline & & 100 & 5.0 & 7.3 & 9.6 & 10.7 & 11.3 & 4.3 & 5.6 & 5.7 & 5.6 & 5.3 \\
\hline & -0.5 & 50 & 4.1 & 6.9 & 9.5 & 10.1 & 10.6 & 4.5 & 5.9 & 6.7 & 6.4 & 5.9 \\
\hline & & 100 & 5.5 & 7.7 & 10.8 & 11.6 & 11.8 & 4.9 & 5.5 & 5.6 & 5.5 & 5.2 \\
\hline & 0.5 & 50 & 3.7 & 6.2 & 8.7 & 9.0 & 8.8 & 4.1 & 5.4 & 5.9 & 5.7 & 5.0 \\
\hline & & 100 & 5.5 & 7.4 & 10.1 & 11.4 & 11.2 & 5.2 & 5.2 & 5.4 & 5.3 & 4.9 \\
\hline & 0.8 & 50 & 5.0 & 6.8 & 7.9 & 8.7 & 9.1 & 5.1 & 5.7 & 4.8 & 5.0 & 4.9 \\
\hline & & 100 & 5.2 & 7.4 & 9.2 & 10.0 & 10.0 & 4.8 & 5.5 & 4.8 & 5.1 & 5.0 \\
\hline \multirow[t]{4}{*}{$\mathrm{MA}(2)$} & -0.5 & 50 & 9.5 & 11.2 & 8.7 & 12.2 & 16.8 & 10.2 & 9.7 & 6.6 & 8.4 & 10.9 \\
\hline & & 100 & 9.3 & 10.1 & 9.7 & 12.6 & 15.7 & 8.2 & 7.5 & 5.7 & 6.7 & 7.9 \\
\hline & 0.5 & 50 & 4.1 & 5.9 & 14.4 & 14.5 & 13.6 & 4.5 & 5.5 & 10.4 & 9.5 & 8.5 \\
\hline & & 100 & 4.4 & 6.8 & 14.1 & 14.1 & 13.0 & 4.1 & 5.0 & 8.2 & 7.3 & 6.1 \\
\hline
\end{tabular}


Table 4. Empirical size of conventional and wild bootstrap HEGY tests using Step 2a of Algorithm 1. Local GLS de-trending. Weakly dependent shocks.

\begin{tabular}{|c|c|c|c|c|c|c|c|c|c|c|c|c|}
\hline & $\Phi / \Theta$ & $N$ & $t_{0}$ & $t_{2}$ & $F_{1}$ & $F_{12}$ & $F_{012}$ & $t_{0}^{*}$ & $t_{2}^{*}$ & $F_{1}^{*}$ & $F_{12}^{*}$ & $F_{012}^{*}$ \\
\hline \multicolumn{13}{|c|}{ Homoskedasticity } \\
\hline \multirow{8}{*}{$\operatorname{AR}(4)$} & -0.8 & 50 & 3.5 & 7.4 & 6.2 & 6.6 & 6.2 & 3.1 & 4.1 & 4.4 & 4.3 & 3.6 \\
\hline & & 100 & 4.3 & 6.6 & 5.6 & 5.3 & 5.6 & 4.1 & 4.5 & 4.8 & 4.4 & 4.2 \\
\hline & -0.5 & 50 & 4.4 & 8.2 & 5.7 & 6.7 & 7.0 & 3.5 & 4.1 & 4.6 & 4.0 & 4.1 \\
\hline & & 100 & 4.9 & 6.5 & 5.3 & 5.1 & 5.4 & 4.6 & 5.2 & 4.9 & 4.7 & 4.6 \\
\hline & 0.5 & 50 & 3.8 & 6.8 & 9.0 & 9.1 & 7.6 & 2.3 & 3.5 & 7.2 & 6.1 & 4.0 \\
\hline & & 100 & 5.2 & 6.9 & 5.8 & 5.8 & 6.3 & 5.0 & 4.6 & 4.6 & 4.4 & 4.7 \\
\hline & 0.8 & 50 & 6.5 & 8.7 & 6.9 & 8.1 & 9.6 & 5.1 & 5.1 & 5.6 & 5.3 & 5.5 \\
\hline & & 100 & 5.4 & 7.0 & 5.7 & 6.1 & 6.6 & 4.9 & 5.1 & 5.3 & 5.3 & 5.0 \\
\hline \multirow[t]{4}{*}{$\operatorname{MA}(2)$} & -0.5 & 50 & 12.1 & 15.0 & 6.6 & 11.0 & 16.3 & 9.8 & 9.9 & 5.0 & 7.4 & 10.7 \\
\hline & & 100 & 9.5 & 10.2 & 5.2 & 7.1 & 10.1 & 8.1 & 7.7 & 4.8 & 6.0 & 7.8 \\
\hline & 0.5 & 50 & 5.1 & 8.2 & 8.3 & 8.8 & 9.2 & 3.9 & 4.5 & 6.4 & 6.3 & 5.5 \\
\hline & & 100 & 4.0 & 5.9 & 6.3 & 6.0 & 5.5 & 3.7 & 4.5 & 5.5 & 5.1 & 4.3 \\
\hline \multicolumn{13}{|c|}{ Periodic heteroskedasticity, Case 2} \\
\hline \multirow[t]{8}{*}{$\operatorname{AR}(4)$} & -0.8 & 50 & 5.1 & 8.8 & 9.9 & 13.4 & 12.9 & 4.5 & 5.3 & 5.7 & 5.6 & 5.5 \\
\hline & & 100 & 4.8 & 7.3 & 9.5 & 12.3 & 12.1 & 4.4 & 5.5 & 5.2 & 5.4 & 5.4 \\
\hline & -0.5 & 50 & 5.8 & 8.8 & 9.9 & 13.2 & 13.4 & 5.4 & 5.3 & 5.7 & 5.5 & 5.8 \\
\hline & & 100 & 5.5 & 6.9 & 9.3 & 12.1 & 11.9 & 5.2 & 5.2 & 5.3 & 5.3 & 5.6 \\
\hline & 0.5 & 50 & 4.9 & 7.9 & 10.7 & 13.4 & 13.0 & 4.0 & 4.3 & 6.2 & 5.8 & 5.4 \\
\hline & & 100 & 5.7 & 7.2 & 9.9 & 12.7 & 12.3 & 5.3 & 4.8 & 5.3 & 5.1 & 5.1 \\
\hline & 0.8 & 50 & 6.9 & 9.2 & 10.0 & 13.3 & 13.7 & 6.1 & 5.6 & 6.1 & 6.1 & 6.1 \\
\hline & & 100 & 5.9 & 7.2 & 9.6 & 12.2 & 12.3 & 5.4 & 5.1 & 5.6 & 5.4 & 5.4 \\
\hline \multirow[t]{4}{*}{$\mathrm{MA}(2)$} & -0.5 & 50 & 9.5 & 11.4 & 9.6 & 14.0 & 16.2 & 8.4 & 7.4 & 5.6 & 6.3 & 7.1 \\
\hline & & 100 & 7.1 & 8.2 & 8.8 & 12.4 & 13.0 & 7.1 & 6.1 & 5.2 & 5.3 & 5.8 \\
\hline & 0.5 & 50 & 5.2 & 8.4 & 12.3 & 15.3 & 14.9 & 4.6 & 5.3 & 8.2 & 7.2 & 6.7 \\
\hline & & 100 & 4.9 & 6.4 & 10.3 & 12.7 & 12.2 & 4.4 & 4.8 & 6.2 & 5.8 & 5.6 \\
\hline \multicolumn{13}{|c|}{ Single volatility shift, $\delta=3, \tau=0.8$} \\
\hline \multirow[t]{8}{*}{$\operatorname{AR}(4)$} & -0.8 & 50 & 4.5 & 8.2 & 4.8 & 5.9 & 6.7 & 3.6 & 4.2 & 4.9 & 4.6 & 4.0 \\
\hline & & 100 & 5.0 & 7.1 & 4.6 & 4.9 & 5.6 & 3.9 & 5.0 & 4.7 & 4.6 & 4.1 \\
\hline & -0.5 & 50 & 5.1 & 8.7 & 4.9 & 6.2 & 7.3 & 3.8 & 4.6 & 5.0 & 4.8 & 4.2 \\
\hline & & 100 & 5.6 & 7.3 & 4.4 & 5.0 & 5.8 & 4.2 & 4.7 & 4.7 & 4.6 & 4.6 \\
\hline & 0.5 & 50 & 4.9 & 7.9 & 5.3 & 6.1 & 6.4 & 3.5 & 3.6 & 4.9 & 4.2 & 3.6 \\
\hline & & 100 & 5.8 & 7.2 & 4.2 & 4.8 & 5.8 & 4.6 & 5.0 & 4.5 & 4.5 & 4.4 \\
\hline & 0.8 & 50 & 6.7 & 8.5 & 4.3 & 5.6 & 7.4 & 4.5 & 4.4 & 4.3 & 4.0 & 4.3 \\
\hline & & 100 & 5.8 & 7.0 & 3.5 & 4.5 & 5.6 & 5.0 & 4.4 & 4.1 & 4.1 & 4.5 \\
\hline \multirow[t]{4}{*}{$\mathrm{MA}(2)$} & -0.5 & 50 & 13.3 & 15.4 & 4.9 & 9.6 & 16.5 & 10.2 & 9.6 & 4.4 & 7.1 & 10.8 \\
\hline & & 100 & 9.6 & 11.0 & 4.2 & 6.6 & 10.3 & 8.5 & 7.2 & 4.4 & 5.7 & 7.9 \\
\hline & 0.5 & 50 & 5.8 & 8.5 & 7.3 & 8.4 & 9.6 & 4.2 & 4.6 & 7.5 & 6.7 & 5.8 \\
\hline & & 100 & 4.6 & 6.5 & 5.6 & 5.2 & 5.6 & 3.5 & 4.4 & 5.9 & 5.5 & 4.4 \\
\hline
\end{tabular}


Table 4. Continued.

\begin{tabular}{|c|c|c|c|c|c|c|c|c|c|c|c|c|}
\hline & $\Phi / \Theta$ & $N$ & $t_{0}$ & $t_{2}$ & $F_{1}$ & $F_{12}$ & $F_{012}$ & $t_{0}^{*}$ & $t_{2}^{*}$ & $F_{1}^{*}$ & $F_{12}^{*}$ & $F_{012}^{*}$ \\
\hline \multicolumn{13}{|c|}{ Single volatility shift, $\delta=3, \tau=0.2$} \\
\hline \multirow[t]{8}{*}{$\operatorname{AR}(4)$} & -0.8 & 50 & 9.0 & 12.6 & 6.7 & 9.0 & 11.8 & 6.1 & 7.1 & 7.2 & 7.9 & 7.7 \\
\hline & & 100 & 8.5 & 10.3 & 5.3 & 7.4 & 8.8 & 5.1 & 5.8 & 6.5 & 6.6 & 6.0 \\
\hline & -0.5 & 50 & 10.5 & 13.2 & 6.6 & 9.7 & 13.1 & 6.6 & 7.0 & 7.4 & 7.7 & 7.9 \\
\hline & & 100 & 9.6 & 9.8 & 5.2 & 6.7 & 9.3 & 5.7 & 5.9 & 6.2 & 6.2 & 6.2 \\
\hline & 0.5 & 50 & 8.6 & 10.2 & 5.0 & 6.7 & 8.8 & 5.0 & 5.2 & 5.0 & 5.1 & 4.9 \\
\hline & & 100 & 8.7 & 9.1 & 4.4 & 5.9 & 8.2 & 5.6 & 5.6 & 5.2 & 5.3 & 5.4 \\
\hline & 0.8 & 50 & 8.0 & 8.0 & 3.8 & 5.1 & 7.2 & 4.8 & 3.8 & 3.7 & 3.6 & 4.0 \\
\hline & & 100 & 7.2 & 7.7 & 3.1 & 4.2 & 6.0 & 4.1 & 4.3 & 3.6 & 3.6 & 3.8 \\
\hline \multirow[t]{4}{*}{$\mathrm{MA}(2)$} & -0.5 & 50 & 19.4 & 20.7 & 5.6 & 13.2 & 22.1 & 13.5 & 13.2 & 6.0 & 10.5 & 15.2 \\
\hline & & 100 & 14.6 & 14.9 & 4.5 & 8.9 & 14.7 & 10.3 & 9.4 & 5.6 & 8.1 & 10.9 \\
\hline & 0.5 & 50 & 9.6 & 11.7 & 10.2 & 12.6 & 15.2 & 6.0 & 6.5 & 10.3 & 9.8 & 8.8 \\
\hline & & 100 & 7.6 & 8.5 & 7.1 & 7.5 & 8.7 & 4.7 & 5.1 & 8.0 & 7.3 & 6.4 \\
\hline \multicolumn{13}{|c|}{ Single periodic volatility shift, $\delta=3, \tau=0.2$, Case 3} \\
\hline \multirow[t]{8}{*}{$\operatorname{AR}(4)$} & -0.8 & 50 & 4.0 & 8.0 & 4.7 & 5.8 & 6.1 & 3.5 & 4.9 & 4.8 & 4.8 & 4.1 \\
\hline & & 100 & 4.6 & 6.3 & 4.6 & 4.6 & 5.5 & 4.3 & 4.7 & 4.8 & 4.6 & 4.3 \\
\hline & -0.5 & 50 & 5.0 & 8.6 & 4.5 & 5.9 & 7.0 & 3.9 & 5.0 & 5.2 & 4.7 & 4.6 \\
\hline & & 100 & 5.8 & 6.5 & 4.3 & 4.8 & 5.8 & 4.7 & 5.0 & 5.5 & 5.5 & 5.1 \\
\hline & 0.5 & 50 & 4.5 & 7.3 & 6.0 & 6.5 & 7.0 & 3.5 & 3.9 & 5.8 & 5.5 & 4.3 \\
\hline & & 100 & 5.4 & 6.9 & 4.2 & 4.8 & 5.8 & 5.1 & 4.7 & 4.8 & 5.0 & 5.0 \\
\hline & 0.8 & 50 & 6.6 & 8.6 & 4.3 & 5.7 & 8.3 & 5.3 & 5.0 & 4.8 & 4.8 & 5.2 \\
\hline & & 100 & 5.2 & 6.3 & 4.4 & 4.9 & 5.8 & 4.5 & 4.7 & 5.2 & 5.2 & 4.9 \\
\hline \multirow[t]{4}{*}{$\mathrm{MA}(2)$} & -0.5 & 50 & 13.0 & 15.8 & 4.4 & 9.4 & 16.4 & 10.8 & 10.8 & 4.8 & 8.3 & 11.8 \\
\hline & & 100 & 9.8 & 10.5 & 4.0 & 6.2 & 10.3 & 8.8 & 7.6 & 4.8 & 6.3 & 8.2 \\
\hline & 0.5 & 50 & 5.4 & 7.9 & 8.0 & 8.7 & 9.7 & 4.3 & 4.8 & 8.6 & 7.4 & 6.3 \\
\hline & & 100 & 4.2 & 6.2 & 6.1 & 5.8 & 5.8 & 4.0 & 4.5 & 7.1 & 6.7 & 5.3 \\
\hline
\end{tabular}


Table 5. Finite sample size-adjusted power of conventional and wild bootstrap HEGY tests using Step 2a of Algorithm 1. OLS de-trending.

\begin{tabular}{|c|c|c|c|c|c|c|c|c|c|c|c|c|c|c|}
\hline$\Sigma$ & $\delta$ & $\tau$ & $-c$ & $N$ & $t_{0}$ & $t_{2}$ & $F_{1}$ & $F_{12}$ & $F_{012}$ & $t_{0}^{*}$ & $t_{2}^{*}$ & $F_{1}^{*}$ & $F_{12}^{*}$ & $F_{012}^{*}$ \\
\hline \multicolumn{15}{|c|}{ Homoskedasticity } \\
\hline & & & \multirow[t]{2}{*}{3.75} & 50 & 6.2 & 14.0 & 21.2 & 27.8 & 24.4 & 6.0 & 13.6 & 21.7 & 27.9 & 24.4 \\
\hline & & & & 100 & 6.4 & 14.6 & 22.1 & 29.6 & 25.3 & 6.4 & 14.5 & 21.5 & 29.3 & 25.6 \\
\hline & & & \multirow[t]{2}{*}{7} & 50 & 12.4 & 30.7 & 48.1 & 63.2 & 60.2 & 12.1 & 29.9 & 48.5 & 63.2 & 60.0 \\
\hline & & & & 100 & 12.2 & 29.8 & 48.8 & 64.5 & 61.5 & 12.1 & 29.8 & 48.7 & 63.9 & 61.2 \\
\hline & & & \multirow[t]{2}{*}{13.5} & 50 & 39.3 & 72.0 & 90.7 & 96.1 & 96.0 & 38.3 & 71.3 & 91.2 & 96.4 & 96.4 \\
\hline & & & & 100 & 36.1 & 69.6 & 92.3 & 98.0 & 98.1 & 35.9 & 69.0 & 92.1 & 98.0 & 98.2 \\
\hline \multicolumn{15}{|c|}{ Periodic heteroskedasticity } \\
\hline \multirow[t]{6}{*}{ Case 1} & & & 3.75 & 50 & 6.9 & 15.2 & 22.3 & 28.2 & 24.5 & 6.8 & 14.9 & 22.1 & 27.9 & 23.9 \\
\hline & & & & 100 & 7.8 & 15.3 & 22.2 & 29.1 & 24.9 & 7.6 & 15.4 & 22.2 & 28.9 & 25.0 \\
\hline & & & 7 & 50 & 12.8 & 31.5 & 45.6 & 59.0 & 55.3 & 13.0 & 31.1 & 44.4 & 58.9 & 54.1 \\
\hline & & & & 100 & 13.5 & 30.6 & 45.7 & 60.2 & 54.8 & 13.2 & 30.7 & 45.0 & 59.6 & 54.5 \\
\hline & & & 13.5 & 50 & 39.8 & 71.5 & 85.3 & 94.5 & 93.6 & 39.6 & 71.1 & 85.2 & 94.5 & 93.5 \\
\hline & & & & 100 & 37.1 & 69.6 & 86.0 & 95.7 & 94.9 & 36.6 & 69.7 & 85.6 & 95.8 & 94.9 \\
\hline \multirow[t]{6}{*}{ Case 2} & & & 3.75 & 50 & 8.8 & 17.0 & 16.7 & 17.1 & 16.4 & 8.7 & 16.3 & 16.7 & 16.6 & 16.1 \\
\hline & & & & 100 & 8.4 & 15.7 & 15.7 & 16.0 & 15.7 & 8.3 & 15.7 & 16.0 & 15.9 & 15.8 \\
\hline & & & 7 & 50 & 15.6 & 33.5 & 33.4 & 33.9 & 31.8 & 15.8 & 32.1 & 33.0 & 32.7 & 31.3 \\
\hline & & & & 100 & 15.3 & 31.9 & 32.3 & 32.6 & 31.2 & 14.9 & 31.4 & 32.2 & 32.0 & 30.7 \\
\hline & & & 13.5 & 50 & 42.8 & 71.7 & 71.6 & 72.0 & 69.7 & 43.0 & 70.2 & 70.8 & 70.9 & 69.1 \\
\hline & & & & 100 & 39.8 & 69.9 & 70.5 & 70.9 & 68.9 & 39.3 & 69.7 & 70.3 & 70.1 & 68.1 \\
\hline \multicolumn{15}{|c|}{ Single volatility shift } \\
\hline \multirow[t]{24}{*}{ Case 1} & 0.33 & 0.2 & 3.75 & 50 & 6.2 & 14.0 & 21.2 & 27.8 & 24.4 & 6.0 & 13.6 & 21.7 & 27.9 & 24.4 \\
\hline & & & & 100 & 6.4 & 14.6 & 22.1 & 29.6 & 25.3 & 6.4 & 14.5 & 21.5 & 29.3 & 25.6 \\
\hline & & & 7 & 50 & 12.4 & 30.7 & 48.1 & 63.2 & 60.2 & 12.1 & 29.9 & 48.5 & 63.2 & 60.0 \\
\hline & & & & 100 & 12.2 & 29.8 & 48.8 & 64.5 & 61.5 & 12.1 & 29.8 & 48.7 & 63.9 & 61.2 \\
\hline & & & 13.5 & 50 & 39.3 & 72.0 & 90.7 & 96.1 & 96.0 & 38.3 & 71.3 & 91.2 & 96.4 & 96.4 \\
\hline & & & & 100 & 36.1 & 69.6 & 92.3 & 98.0 & 98.1 & 35.9 & 69.0 & 92.1 & 98.0 & 98.2 \\
\hline & & 0.8 & 3.75 & 50 & 7.2 & 11.4 & 14.7 & 17.6 & 16.7 & 7.0 & 11.1 & 14.6 & 17.1 & 17.1 \\
\hline & & & & 100 & 7.7 & 11.8 & 14.2 & 17.0 & 17.4 & 7.8 & 11.5 & 14.2 & 16.7 & 16.8 \\
\hline & & & 7 & 50 & 13.1 & 21.3 & 29.3 & 37.3 & 38.6 & 13.2 & 21.3 & 28.9 & 36.2 & 38.7 \\
\hline & & & & 100 & 12.9 & 21.0 & 28.0 & 36.2 & 39.6 & 13.4 & 20.8 & 28.0 & 35.5 & 38.1 \\
\hline & & & 13.5 & 50 & 32.4 & 47.8 & 63.9 & 75.8 & 79.0 & 32.6 & 48.1 & 64.1 & 75.8 & 79.7 \\
\hline & & & & 100 & 30.7 & 47.0 & 63.1 & 77.5 & 83.1 & 31.4 & 46.3 & 63.3 & 77.3 & 82.4 \\
\hline & 3 & 0.2 & 3.75 & 50 & 7.9 & 2.4 & 2.3 & 1.8 & 2.6 & 6.8 & 1.9 & 1.8 & 1.5 & 2.3 \\
\hline & & & & 100 & 6.2 & 1.5 & 1.4 & 0.8 & 1.4 & 5.4 & 1.5 & 1.4 & 0.9 & 1.5 \\
\hline & & & 7 & 50 & 8.5 & 4.5 & 4.7 & 4.9 & 6.7 & 7.1 & 3.5 & 3.8 & 3.8 & 4.8 \\
\hline & & & & 100 & 6.4 & 2.9 & 2.8 & 2.6 & 3.6 & 5.4 & 2.5 & 2.6 & 2.5 & 3.3 \\
\hline & & & 13.5 & 50 & 19.7 & 17.3 & 26.1 & 34.8 & 42.5 & 16.3 & 14.0 & 20.2 & 25.3 & 29.4 \\
\hline & & & & 100 & 14.3 & 11.2 & 16.3 & 21.7 & 29.1 & 12.0 & 9.8 & 13.8 & 18.5 & 23.1 \\
\hline & & 0.8 & 3.75 & 50 & 5.4 & 7.7 & 11.4 & 14.5 & 13.6 & 5.2 & 8.0 & 11.0 & 13.6 & 12.5 \\
\hline & & & & 100 & 5.2 & 8.2 & 10.9 & 13.3 & 12.2 & 5.2 & 7.7 & 10.5 & 13.1 & 12.1 \\
\hline & & & 7 & 50 & 9.3 & 15.4 & 27.1 & 37.8 & 37.7 & 9.1 & 15.4 & 25.9 & 34.5 & 34.4 \\
\hline & & & & 100 & 8.8 & 15.1 & 25.2 & 35.5 & 35.1 & 8.7 & 14.6 & 24.4 & 33.8 & 33.5 \\
\hline & & & 13.5 & 50 & 30.3 & 50.3 & 80.4 & 91.5 & 92.2 & 29.0 & 48.9 & 77.1 & 88.8 & 89.4 \\
\hline & & & & 100 & 26.6 & 46.5 & 77.2 & 91.1 & 92.2 & 25.5 & 44.8 & 74.8 & 89.2 & 90.7 \\
\hline
\end{tabular}


Table 5. Continued.

\begin{tabular}{|c|c|c|c|c|c|c|c|c|c|c|c|c|c|c|}
\hline$\Sigma$ & $\delta$ & $\tau$ & $-c$ & $N$ & $t_{0}$ & $t_{2}$ & $F_{1}$ & $F_{12}$ & $F_{012}$ & $t_{0}^{*}$ & $t_{2}^{*}$ & $F_{1}^{*}$ & $F_{12}^{*}$ & $F_{012}^{*}$ \\
\hline \multicolumn{15}{|c|}{ Single periodic volatility shift } \\
\hline \multirow[t]{24}{*}{ Case 3} & 0.33 & 0.2 & 3.75 & 50 & 6.2 & 14.0 & 21.2 & 27.8 & 24.4 & 6.0 & 13.6 & 21.7 & 27.9 & 24.4 \\
\hline & & & & 100 & 6.4 & 14.6 & 22.1 & 29.6 & 25.3 & 6.4 & 14.5 & 21.5 & 29.3 & 25.6 \\
\hline & & & 7 & 50 & 12.4 & 30.7 & 48.1 & 63.2 & 60.2 & 12.1 & 29.9 & 48.5 & 63.2 & 60.0 \\
\hline & & & & 100 & 12.2 & 29.8 & 48.8 & 64.5 & 61.5 & 12.1 & 29.8 & 48.7 & 63.9 & 61.2 \\
\hline & & & 13.5 & 50 & 39.3 & 72.0 & 90.7 & 96.1 & 96.0 & 38.3 & 71.3 & 91.2 & 96.4 & 96.4 \\
\hline & & & & 100 & 36.1 & 69.6 & 92.3 & 98.0 & 98.1 & 35.9 & 69.0 & 92.1 & 98.0 & 98.2 \\
\hline & & 0.8 & 3.75 & 50 & 7.2 & 11.4 & 14.7 & 17.6 & 16.7 & 7.0 & 11.1 & 14.6 & 17.1 & 17.1 \\
\hline & & & & 100 & 7.7 & 11.8 & 14.2 & 17.0 & 17.4 & 7.8 & 11.5 & 14.2 & 16.7 & 16.8 \\
\hline & & & 7 & 50 & 13.1 & 21.3 & 29.3 & 37.3 & 38.6 & 13.2 & 21.3 & 28.9 & 36.2 & 38.7 \\
\hline & & & & 100 & 12.9 & 21.0 & 28.0 & 36.2 & 39.6 & 13.4 & 20.8 & 28.0 & 35.5 & 38.1 \\
\hline & & & 13.5 & 50 & 32.4 & 47.8 & 63.9 & 75.8 & 79.0 & 32.6 & 48.1 & 64.1 & 75.8 & 79.7 \\
\hline & & & & 100 & 30.7 & 47.0 & 63.1 & 77.5 & 83.1 & 31.4 & 46.3 & 63.3 & 77.3 & 82.4 \\
\hline & 3 & 0.2 & 3.75 & 50 & 7.9 & 2.4 & 2.3 & 1.8 & 2.6 & 6.8 & 1.9 & 1.8 & 1.5 & 2.3 \\
\hline & & & & 100 & 6.2 & 1.5 & 1.4 & 0.8 & 1.4 & 5.4 & 1.5 & 1.4 & 0.9 & 1.5 \\
\hline & & & 7 & 50 & 8.5 & 4.5 & 4.7 & 4.9 & 6.7 & 7.1 & 3.5 & 3.8 & 3.8 & 4.8 \\
\hline & & & & 100 & 6.4 & 2.9 & 2.8 & 2.6 & 3.6 & 5.4 & 2.5 & 2.6 & 2.5 & 3.3 \\
\hline & & & 13.5 & 50 & 19.7 & 17.3 & 26.1 & 34.8 & 42.5 & 16.3 & 14.0 & 20.2 & 25.3 & 29.4 \\
\hline & & & & 100 & 14.3 & 11.2 & 16.3 & 21.7 & 29.1 & 12.0 & 9.8 & 13.8 & 18.5 & 23.1 \\
\hline & & 0.8 & 3.75 & 50 & 5.4 & 7.7 & 11.4 & 14.5 & 13.6 & 5.2 & 8.0 & 11.0 & 13.6 & 12.5 \\
\hline & & & & 100 & 5.2 & 8.2 & 10.9 & 13.3 & 12.2 & 5.2 & 7.7 & 10.5 & 13.1 & 12.1 \\
\hline & & & 7 & 50 & 9.3 & 15.4 & 27.1 & 37.8 & 37.7 & 9.1 & 15.4 & 25.9 & 34.5 & 34.4 \\
\hline & & & & 100 & 8.8 & 15.1 & 25.2 & 35.5 & 35.1 & 8.7 & 14.6 & 24.4 & 33.8 & 33.5 \\
\hline & & & 13.5 & 50 & 30.3 & 50.3 & 80.4 & 91.5 & 92.2 & 29.0 & 48.9 & 77.1 & 88.8 & 89.4 \\
\hline & & & & 100 & 26.6 & 46.5 & 77.2 & 91.1 & 92.2 & 25.5 & 44.8 & 74.8 & 89.2 & 90.7 \\
\hline \multirow[t]{12}{*}{ Case 4} & & 0.2 & 3.75 & 50 & 8.7 & 3.0 & 2.3 & 2.3 & 3.4 & 7.0 & 2.4 & 2.2 & 1.8 & 2.5 \\
\hline & & & & 100 & 6.4 & 1.7 & 1.3 & 1.0 & 1.6 & 5.9 & 1.6 & 1.3 & 1.1 & 1.6 \\
\hline & & & 7 & 50 & 9.9 & 5.3 & 5.2 & 6.1 & 7.6 & 8.0 & 4.5 & 4.6 & 4.5 & 5.8 \\
\hline & & & & 100 & 6.6 & 3.3 & 2.8 & 2.9 & 3.9 & 6.4 & 2.8 & 2.8 & 2.8 & 3.5 \\
\hline & & & 13.5 & 50 & 21.7 & 19.0 & 23.7 & 33.8 & 38.1 & 18.3 & 16.0 & 19.2 & 24.9 & 27.6 \\
\hline & & & & 100 & 14.4 & 12.6 & 14.9 & 21.2 & 25.9 & 13.5 & 10.7 & 13.1 & 17.2 & 20.5 \\
\hline & & 0.8 & 3.75 & 50 & 5.9 & 9.0 & 12.4 & 15.3 & 14.1 & 5.6 & 8.4 & 11.3 & 13.7 & 12.5 \\
\hline & & & & 100 & 6.2 & 8.7 & 11.2 & 13.2 & 13.0 & 6.0 & 8.2 & 10.8 & 13.0 & 12.6 \\
\hline & & & 7 & 50 & 9.7 & 17.5 & 26.7 & 36.7 & 35.2 & 9.3 & 16.5 & 23.9 & 32.0 & 31.0 \\
\hline & & & & 100 & 9.8 & 16.7 & 23.1 & 32.6 & 31.9 & 9.2 & 15.4 & 21.8 & 30.8 & 30.1 \\
\hline & & & 13.5 & 50 & 31.0 & 52.3 & 71.6 & 87.3 & 86.6 & 29.9 & 49.5 & 66.0 & 81.6 & 80.9 \\
\hline & & & & 100 & 27.9 & 47.6 & 65.3 & 83.8 & 83.6 & 26.1 & 45.3 & 62.5 & 80.6 & 80.7 \\
\hline
\end{tabular}


Table 6. Finite sample size-adjusted power of conventional and wild bootstrap HEGY tests using Step 2a of Algorithm 1. GLS de-trending.

\begin{tabular}{|c|c|c|c|c|c|c|c|c|c|c|c|c|c|c|}
\hline$\Sigma$ & $\delta$ & $\tau$ & $-c$ & $N$ & $t_{0}$ & $t_{2}$ & $F_{1}$ & $F_{12}$ & $F_{012}$ & $t_{0}^{*}$ & $t_{2}^{*}$ & $F_{1}^{*}$ & $F_{12}^{*}$ & $F_{012}^{*}$ \\
\hline \multicolumn{15}{|c|}{ Homoskedasticity } \\
\hline & & & \multirow[t]{2}{*}{3.75} & 50 & 7.6 & 17.4 & 25.8 & 35.2 & 31.1 & 7.3 & 17.4 & 26.0 & 35.2 & 31.1 \\
\hline & & & & 100 & 8.4 & 18.8 & 25.1 & 35.3 & 31.9 & 8.3 & 18.4 & 24.5 & 34.4 & 31.2 \\
\hline & & & \multirow[t]{2}{*}{7} & 50 & 15.5 & 39.1 & 62.5 & 78.9 & 74.4 & 14.9 & 39.3 & 62.6 & 78.4 & 74.3 \\
\hline & & & & 100 & 15.9 & 40.4 & 61.9 & 78.9 & 75.9 & 16.0 & 39.7 & 60.9 & 77.8 & 75.0 \\
\hline & & & \multirow[t]{2}{*}{13.5} & 50 & 46.8 & 82.5 & 96.4 & 98.6 & 98.2 & 45.3 & 82.3 & 96.5 & 98.8 & 98.4 \\
\hline & & & & 100 & 45.2 & 84.0 & 98.0 & 99.7 & 99.6 & 45.0 & 83.4 & 97.7 & 99.7 & 99.5 \\
\hline \multicolumn{15}{|c|}{ Periodic heteroskedasticity } \\
\hline \multirow[t]{6}{*}{ Case 1} & & & 3.75 & 50 & 8.2 & 18.6 & 29.1 & 37.4 & 30.6 & 8.2 & 18.5 & 28.3 & 36.8 & 30.2 \\
\hline & & & & 100 & 9.1 & 18.4 & 26.5 & 35.5 & 30.4 & 9.1 & 18.8 & 26.1 & 35.2 & 30.5 \\
\hline & & & 7 & 50 & 16.0 & 40.3 & 61.3 & 76.5 & 68.6 & 15.9 & 40.0 & 59.4 & 75.6 & 68.2 \\
\hline & & & & 100 & 16.9 & 39.8 & 58.2 & 75.5 & 68.8 & 16.7 & 40.6 & 57.4 & 74.2 & 68.7 \\
\hline & & & 13.5 & 50 & 47.1 & 82.3 & 94.8 & 98.4 & 97.3 & 46.8 & 81.8 & 94.5 & 98.4 & 97.5 \\
\hline & & & & 100 & 46.2 & 83.2 & 95.2 & 99.1 & 98.3 & 45.6 & 83.3 & 94.9 & 99.0 & 98.4 \\
\hline \multirow[t]{6}{*}{ Case 2} & & & 3.75 & 50 & 10.2 & 20.5 & 20.6 & 20.8 & 21.0 & 10.3 & 19.6 & 19.7 & 20.3 & 19.8 \\
\hline & & & & 100 & 10.2 & 19.4 & 18.6 & 19.3 & 19.8 & 10.3 & 19.1 & 17.8 & 18.3 & 18.9 \\
\hline & & & 7 & 50 & 19.3 & 42.3 & 44.7 & 44.1 & 43.1 & 19.2 & 40.8 & 42.8 & 43.1 & 40.9 \\
\hline & & & & 100 & 18.9 & 41.4 & 41.1 & 42.0 & 41.4 & 18.7 & 40.6 & 39.6 & 40.2 & 39.9 \\
\hline & & & 13.5 & 50 & 50.3 & 82.3 & 85.1 & 84.3 & 83.1 & 50.2 & 80.8 & 83.7 & 83.2 & 81.2 \\
\hline & & & & 100 & 48.9 & 83.8 & 84.4 & 84.8 & 83.6 & 48.3 & 83.3 & 83.2 & 83.4 & 82.1 \\
\hline \multicolumn{15}{|c|}{ Single volatility shift } \\
\hline \multirow[t]{24}{*}{ Case 1} & 0.33 & 0.2 & 3.75 & 50 & 7.6 & 17.4 & 25.8 & 35.2 & 31.1 & 7.3 & 17.4 & 26.0 & 35.2 & 31.1 \\
\hline & & & & 100 & 8.4 & 18.8 & 25.1 & 35.3 & 31.9 & 8.3 & 18.4 & 24.5 & 34.4 & 31.2 \\
\hline & & & 7 & 50 & 15.5 & 39.1 & 62.5 & 78.9 & 74.4 & 14.9 & 39.3 & 62.6 & 78.4 & 74.3 \\
\hline & & & & 100 & 15.9 & 40.4 & 61.9 & 78.9 & 75.9 & 16.0 & 39.7 & 60.9 & 77.8 & 75.0 \\
\hline & & & 13.5 & 50 & 46.8 & 82.5 & 96.4 & 98.6 & 98.2 & 45.3 & 82.3 & 96.5 & 98.8 & 98.4 \\
\hline & & & & 100 & 45.2 & 84.0 & 98.0 & 99.7 & 99.6 & 45.0 & 83.4 & 97.7 & 99.7 & 99.5 \\
\hline & & 0.8 & 3.75 & 50 & 8.2 & 17.7 & 18.6 & 23.7 & 23.3 & 8.3 & 17.8 & 18.8 & 23.2 & 23.0 \\
\hline & & & & 100 & 8.9 & 17.6 & 15.5 & 21.3 & 20.3 & 8.8 & 18.5 & 16.1 & 20.1 & 20.8 \\
\hline & & & 7 & 50 & 15.7 & 34.5 & 41.5 & 52.5 & 53.9 & 16.1 & 34.2 & 40.5 & 51.4 & 53.1 \\
\hline & & & & 100 & 17.1 & 34.4 & 37.8 & 50.2 & 52.0 & 17.1 & 35.7 & 37.4 & 48.6 & 51.9 \\
\hline & & & 13.5 & 50 & 39.0 & 64.4 & 77.6 & 87.3 & 88.6 & 39.4 & 64.8 & 77.4 & 87.4 & 89.4 \\
\hline & & & & 100 & 40.5 & 65.8 & 76.3 & 88.9 & 92.1 & 40.4 & 66.9 & 76.2 & 88.4 & 92.2 \\
\hline & 3 & 0.2 & 3.75 & 50 & 9.0 & 23.9 & 49.0 & 58.0 & 48.7 & 9.1 & 23.2 & 47.3 & 56.6 & 46.8 \\
\hline & & & & 100 & 8.4 & 22.7 & 47.5 & 57.2 & 45.5 & 8.3 & 22.7 & 46.7 & 56.6 & 45.0 \\
\hline & & & 7 & 50 & 17.6 & 45.5 & 83.6 & 90.6 & 86.9 & 17.3 & 43.7 & 82.3 & 90.0 & 86.2 \\
\hline & & & & 100 & 15.9 & 45.7 & 82.9 & 91.6 & 87.1 & 16.4 & 45.4 & 81.9 & 91.4 & 86.4 \\
\hline & & & 13.5 & 50 & 46.0 & 80.5 & 99.2 & 99.8 & 99.7 & 45.9 & 80.5 & 99.2 & 99.9 & 99.8 \\
\hline & & & & 100 & 43.0 & 82.2 & 99.4 & 99.9 & 99.9 & 42.8 & 81.7 & 99.5 & 99.9 & 99.9 \\
\hline & & 0.8 & 3.75 & 50 & 6.4 & 20.8 & 39.1 & 53.0 & 41.1 & 6.7 & 20.8 & 39.5 & 52.0 & 40.7 \\
\hline & & & & 100 & 6.7 & 22.5 & 39.7 & 53.5 & 41.6 & 6.7 & 22.2 & 40.0 & 52.9 & 42.1 \\
\hline & & & 7 & 50 & 14.0 & 46.8 & 82.3 & 93.5 & 87.6 & 14.4 & 46.5 & 82.1 & 93.0 & 87.6 \\
\hline & & & & 100 & 14.0 & 50.2 & 83.0 & 94.9 & 89.4 & 14.2 & 48.8 & 82.8 & 94.4 & 89.5 \\
\hline & & & 13.5 & 50 & 47.3 & 88.7 & 99.5 & 99.9 & 99.6 & 47.1 & 88.6 & 99.7 & 99.9 & 99.8 \\
\hline & & & & 100 & 46.7 & 91.9 & 99.8 & 100.0 & 100.0 & 46.7 & 91.1 & 99.8 & 100.0 & 100.0 \\
\hline
\end{tabular}


Table 6. Continued.

\begin{tabular}{|c|c|c|c|c|c|c|c|c|c|c|c|c|c|c|}
\hline$\Sigma$ & $\delta$ & $\tau$ & $-c$ & $N$ & $t_{0}$ & $t_{2}$ & $F_{1}$ & $F_{12}$ & $F_{012}$ & $t_{0}^{*}$ & $t_{2}^{*}$ & $F_{1}^{*}$ & $F_{12}^{*}$ & $F_{012}^{*}$ \\
\hline \multicolumn{15}{|c|}{ Single periodic volatility shift } \\
\hline \multirow[t]{24}{*}{ Case 3} & 0.33 & 0.2 & 3.75 & 50 & 7.6 & 17.4 & 25.8 & 35.2 & 31.1 & 7.3 & 17.4 & 26.0 & 35.2 & 31.1 \\
\hline & & & & 100 & 8.4 & 18.8 & 25.1 & 35.3 & 31.9 & 8.3 & 18.4 & 24.5 & 34.4 & 31.2 \\
\hline & & & 7 & 50 & 15.5 & 39.1 & 62.5 & 78.9 & 74.4 & 14.9 & 39.3 & 62.6 & 78.4 & 74.3 \\
\hline & & & & 100 & 15.9 & 40.4 & 61.9 & 78.9 & 75.9 & 16.0 & 39.7 & 60.9 & 77.8 & 75.0 \\
\hline & & & 13.5 & 50 & 46.8 & 82.5 & 96.4 & 98.6 & 98.2 & 45.3 & 82.3 & 96.5 & 98.8 & 98.4 \\
\hline & & & & 100 & 45.2 & 84.0 & 98.0 & 99.7 & 99.6 & 45.0 & 83.4 & 97.7 & 99.7 & 99.5 \\
\hline & & 0.8 & 3.75 & 50 & 8.2 & 17.7 & 18.6 & 23.7 & 23.3 & 8.3 & 17.8 & 18.8 & 23.2 & 23.0 \\
\hline & & & & 100 & 8.9 & 17.6 & 15.5 & 21.3 & 20.3 & 8.8 & 18.5 & 16.1 & 20.1 & 20.8 \\
\hline & & & 7 & 50 & 15.7 & 34.5 & 41.5 & 52.5 & 53.9 & 16.1 & 34.2 & 40.5 & 51.4 & 53.1 \\
\hline & & & & 100 & 17.1 & 34.4 & 37.8 & 50.2 & 52.0 & 17.1 & 35.7 & 37.4 & 48.6 & 51.9 \\
\hline & & & 13.5 & 50 & 39.0 & 64.4 & 77.6 & 87.3 & 88.6 & 39.4 & 64.8 & 77.4 & 87.4 & 89.4 \\
\hline & & & & 100 & 40.5 & 65.8 & 76.3 & 88.9 & 92.1 & 40.4 & 66.9 & 76.2 & 88.4 & 92.2 \\
\hline & 3 & 0.2 & 3.75 & 50 & 9.0 & 23.9 & 49.0 & 58.0 & 48.7 & 9.1 & 23.2 & 47.3 & 56.6 & 46.8 \\
\hline & & & & 100 & 8.4 & 22.7 & 47.5 & 57.2 & 45.5 & 8.3 & 22.7 & 46.7 & 56.6 & 45.0 \\
\hline & & & 7 & 50 & 17.6 & 45.5 & 83.6 & 90.6 & 86.9 & 17.3 & 43.7 & 82.3 & 90.0 & 86.2 \\
\hline & & & & 100 & 15.9 & 45.7 & 82.9 & 91.6 & 87.1 & 16.4 & 45.4 & 81.9 & 91.4 & 86.4 \\
\hline & & & 13.5 & 50 & 46.0 & 80.5 & 99.2 & 99.8 & 99.7 & 45.9 & 80.5 & 99.2 & 99.9 & 99.8 \\
\hline & & & & 100 & 43.0 & 82.2 & 99.4 & 99.9 & 99.9 & 42.8 & 81.7 & 99.5 & 99.9 & 99.9 \\
\hline & & 0.8 & 3.75 & 50 & 6.4 & 20.8 & 39.1 & 53.0 & 41.1 & 6.7 & 20.8 & 39.5 & 52.0 & 40.7 \\
\hline & & & & 100 & 6.7 & 22.5 & 39.7 & 53.5 & 41.6 & 6.7 & 22.2 & 40.0 & 52.9 & 42.1 \\
\hline & & & 7 & 50 & 14.0 & 46.8 & 82.3 & 93.5 & 87.6 & 14.4 & 46.5 & 82.1 & 93.0 & 87.6 \\
\hline & & & & 100 & 14.0 & 50.2 & 83.0 & 94.9 & 89.4 & 14.2 & 48.8 & 82.8 & 94.4 & 89.5 \\
\hline & & & 13.5 & 50 & 47.3 & 88.7 & 99.5 & 99.9 & 99.6 & 47.1 & 88.6 & 99.7 & 99.9 & 99.8 \\
\hline & & & & 100 & 46.7 & 91.9 & 99.8 & 100.0 & 100.0 & 46.7 & 91.1 & 99.8 & 100.0 & 100.0 \\
\hline \multirow[t]{12}{*}{ Case 4} & & 0.2 & 3.75 & 50 & 9.6 & 24.3 & 42.3 & 53.3 & 42.6 & 9.5 & 23.2 & 42.5 & 52.7 & 41.9 \\
\hline & & & & 100 & 8.8 & 23.3 & 42.1 & 52.8 & 40.6 & 8.7 & 22.6 & 42.3 & 52.0 & 40.7 \\
\hline & & & 7 & 50 & 18.7 & 45.5 & 73.9 & 86.0 & 78.2 & 18.6 & 44.5 & 73.9 & 85.4 & 77.1 \\
\hline & & & & 100 & 17.3 & 46.3 & 73.8 & 87.1 & 77.6 & 17.0 & 45.3 & 73.8 & 86.7 & 77.1 \\
\hline & & & 13.5 & 50 & 47.6 & 80.3 & 96.9 & 99.4 & 98.6 & 47.9 & 80.3 & 97.1 & 99.6 & 98.7 \\
\hline & & & & 100 & 45.1 & 82.5 & 97.1 & 99.7 & 98.8 & 44.1 & 81.8 & 97.0 & 99.6 & 99.0 \\
\hline & & 0.8 & 3.75 & 50 & 6.9 & 21.7 & 39.3 & 52.1 & 39.8 & 7.0 & 21.9 & 38.9 & 50.6 & 38.9 \\
\hline & & & & 100 & 7.4 & 22.7 & 39.2 & 51.1 & 39.3 & 7.5 & 22.4 & 39.0 & 50.8 & 38.7 \\
\hline & & & 7 & 50 & 15.0 & 46.4 & 76.1 & 90.0 & 79.6 & 14.8 & 46.4 & 75.3 & 88.9 & 78.6 \\
\hline & & & & 100 & 15.3 & 49.7 & 75.8 & 90.9 & 81.5 & 15.5 & 49.1 & 75.8 & 90.5 & 80.9 \\
\hline & & & 13.5 & 50 & 48.8 & 88.3 & 98.9 & 99.7 & 99.3 & 48.4 & 88.3 & 99.0 & 99.8 & 99.3 \\
\hline & & & & 100 & 47.0 & 91.4 & 99.3 & 99.9 & 99.8 & 46.6 & 90.8 & 99.1 & 100.0 & 99.7 \\
\hline
\end{tabular}




\section{Supplementary Online Appendix}

to

Wild Bootstrap Seasonal Unit Root Tests for Time Series with Periodic Non-Stationary Volatility 


\section{S.1 Contents}

Section S.1 of this supplement contains mathematical proofs of Lemma 1, Propositions 1 and 2, and Corollary 1. Section S.2 contains additional Monte Carlo results relating to the wild bootstrap HEGY tests which use the seasonal block wild re-sampling scheme outlined in Step 2b of Algorithm 1.

\section{S.2 Mathematical Proofs}

\section{Preliminaries:}

In order to simplify the presentation we assume that the investigator has available sufficient pre-sample values of the data such that (9) can be estimated along the index $S n+s=S+1, \ldots, T$. This does not affect the results which follow and is simply a convenient re-basing of the sample index.

Throughout this appendix we will make use of the following definitions. Let $c_{0}, c_{S / 2}, c_{k}$ and $\tilde{c}_{k}$, $k=1, \ldots, S^{*}$, denote the (mutually orthogonal) $S \times 1$ selection vectors defined as: $c_{0}:=[1,1,1, \ldots, 1]^{\prime}$, $c_{S / 2}:=[1,-1,1,-1 \ldots, 1]^{\prime}, c_{k}:=\left[\cos \left(\omega_{k}[1-S]\right), \cos \left(\omega_{k}[2-S]\right), \ldots, \cos (0)\right]^{\prime}$ and $\tilde{c}_{k}:=\left[\sin \left(\omega_{k}[1-\right.\right.$ $\left.S]), \sin \left(\omega_{k}[2-S]\right), \ldots, \sin (0)\right]^{\prime}$. We also introduce the $S \times S$ circulant matrices: $C_{0}:=\operatorname{circ}[1,1,1, \ldots, 1]$, $C_{S / 2}:=\operatorname{circ}[1,-1,1, \ldots,-1]$ and for $\omega_{i}=2 \pi i / S, C_{i}:=\operatorname{circ}\left[\cos (0), \cos \left(\omega_{i}\right), \cos \left(2 \omega_{i}\right), \ldots, \cos ((S-\right.$ 1) $\left.\left.\omega_{i}\right)\right]$ and $\tilde{C}_{i}:=\operatorname{circ}\left[\sin (0), \sin \left(\omega_{i}\right), \sin \left(2 \omega_{i}\right), \ldots, \sin \left((S-1) \omega_{i}\right)\right], i=1, \ldots, S^{*}$. As discussed in Smith et al. (2009, pp.555-556), these matrices are mutually orthogonal and have the following properties: $C_{0} C_{0}=S C_{0}, C_{S / 2} C_{S / 2}=S C_{S / 2}, C_{j} \tilde{C}_{j}=(S / 2) \tilde{C}_{j}, \tilde{C}_{j} \tilde{C}_{j}=(S / 2) C_{j}, j=1, \ldots, S^{*}$. Notice that $C_{0}=c_{0} c_{0}^{\prime}, C_{S / 2}=c_{S / 2} c_{S / 2}^{\prime}, C_{j}=\mathbf{c}_{j} \mathbf{c}_{j}^{\prime}$ and $\tilde{C}_{j}=\mathbf{c}_{j} \tilde{\mathbf{c}}_{j}^{\prime}$, where

$\mathbf{c}_{k}^{\prime}:=\left[\begin{array}{c}\cos \left(\omega_{k}[1-S]\right), \cos \left(\omega_{k}[2-S]\right), \ldots, \cos (0) \\ \sin \left(\omega_{k}[1-S]\right), \sin \left(\omega_{k}[2-S]\right), \ldots, \sin (0)\end{array}\right], \tilde{\mathbf{c}}_{k}^{\prime}:=\left[\begin{array}{c}-\sin \left(\omega_{k}[1-S]\right),-\sin \left(\omega_{k}[2-S]\right), \ldots,-\sin (0) \\ \cos \left(\omega_{k}[1-S]\right), \cos \left(\omega_{k}[2-S]\right), \ldots, \cos (0)\end{array}\right]$.

Finally, define $\mathbf{X}_{j, n}:=\left[x_{j, S n-(S-1)}, x_{j, S n-(S-2)}, \ldots, x_{j, S n}\right]^{\prime}, j=0, S / 2, \mathbf{X}_{j, n}^{\alpha}:=\left[x_{j, S n-(S-1)}^{\alpha}\right.$, $\left.x_{j, S n-(S-2)}^{\alpha}, \ldots, x_{j, S n}^{\alpha}\right]^{\prime}$ and $\mathbf{X}_{j, n}^{\beta}:=\left[x_{j, S n-(S-1)}^{\beta}, x_{j, S n-(S-2)}^{\beta}, \ldots, x_{j, S n}^{\beta}\right]^{\prime}, j=1, \ldots, S^{*}$. With $\mathbf{X}_{n}$ defined as in (10), the following identities hold: $\mathbf{X}_{j, n}=C_{j} \mathbf{X}_{n}, j=0, S / 2, \mathbf{X}_{j, n}^{\alpha}=C_{j} \mathbf{X}_{n}, j=0, \ldots, S^{*}$ and $\mathbf{X}_{j, n}^{\beta}=\tilde{C}_{j} \mathbf{X}_{n}, j=1, \ldots, S^{*}$.

\section{Proof of Lemma 1:}

Under Assumption 1, by Lemma 1 in Boswijk et al. (2016) it holds that

$$
N^{-1 / 2} \sum_{n=2}^{\lfloor\cdot N\rfloor} \mathcal{E}_{n} \Rightarrow \mathbf{M}(\cdot)
$$

Consider now the representation given in (11). By the (multivariate) Beveridge-Nelson decomposition, we have that $U_{n}=\mathbf{\Psi}(1) \mathcal{E}_{n}+\tilde{\mathcal{E}}_{n-1}-\tilde{\mathcal{E}}_{n}$, where $\tilde{\mathcal{E}}_{n}=\tilde{\mathbf{\Psi}}(L) \mathcal{E}_{n}=\sum_{j=0}^{\infty} \tilde{\boldsymbol{\Psi}}_{j} \mathcal{E}_{n-j}, \tilde{\mathbf{\Psi}}_{j}:=\sum_{k=j+1}^{\infty} \boldsymbol{\Psi}_{k}$. By summing over $n$ and multiplying by $N^{-1 / 2}$ we obtain, for any $r \in[0,1]$,

$$
N^{-1 / 2} \sum_{n=2}^{\lfloor r N\rfloor} U_{n}=\Psi(1) N^{-1 / 2} \sum_{n=2}^{\lfloor r N\rfloor} \mathcal{E}_{n}+N^{-1 / 2}\left(\tilde{\mathcal{E}}_{0}-\tilde{\mathcal{E}}_{\lfloor r N\rfloor}\right) .
$$


Under Assumptions 1 and 2, the second term on the right hand side of (S.2) is of $O_{p}\left(N^{-1}\right)$ uniformly in $r \in[0,1]$, provided the sequence $\left\{\tilde{\boldsymbol{\Psi}}_{j}\right\}$ is absolutely summable, i.e. if $\sum_{j=0}^{\infty}\left\|\tilde{\boldsymbol{\Psi}}_{j}\right\|<\infty$. But absolute summability trivially holds since $\sum_{j=0}^{\infty} j|| \boldsymbol{\Psi}_{j}||<\infty$ is implied by $\sum_{j=0}^{\infty} j\left|\psi_{j}\right|<\infty$, which holds under Assumption 2; see e.g. Burridge and Taylor (2001b, pp.374, 377). Finally, the result in (12) follows from (S.1) and the continuous mapping theorem (CMT).

\section{Proof of Proposition 1:}

To prove Proposition 1 we initially proceed as in Burridge and Taylor (2001a) and re-write the regression (9) in matrix form as $\mathbf{y}=\left[\mathbf{Y}: \mathbf{Z}_{p}\right] \boldsymbol{\beta}+\mathbf{u}$, where $\mathbf{y}:=\left[\Delta_{S} x_{S+1}, \ldots, \Delta_{S} x_{S n}\right]^{\prime}, \mathbf{Y}$ is the matrix containing regressors $x_{0, S n+s-1}, x_{k, S n+s-1}^{\alpha}$ and $x_{k, S n+s-1}^{\beta}, k=1, \ldots, S^{*}$, and $x_{S / 2, S n+s-1}$, i.e. $\mathbf{Y}:=\left[\mathbf{y}_{0}, \mathbf{y}_{1}^{\alpha}, \mathbf{y}_{1}^{\beta}, \ldots, \mathbf{y}_{S^{*}}^{\alpha}, \mathbf{y}_{S^{*}}^{\beta}, \mathbf{y}_{S / 2}\right] ; \mathbf{Z}_{p}$ is the matrix containing $p$ lags of seasonal differences, $\left\{\Delta_{S} x_{S n+s-j}\right\}_{j=1}^{p}, \boldsymbol{\beta}:=\left[\pi_{0}, \pi_{\alpha, 1}, \ldots, \pi_{\alpha, S^{*}}, \pi_{\beta, 1}, \ldots, \pi_{\beta, S^{*}}, \pi_{S / 2}\right.$, $\left.\phi_{1}, \ldots, \phi_{p}\right]^{\prime}$, and $\mathbf{u}:=\left[u_{p+1}, \ldots, u_{S n}\right]^{\prime}$. Then,

$$
\mathbf{D}_{N}^{-1}[\hat{\boldsymbol{\beta}}-\boldsymbol{\beta}]=\left[\begin{array}{cc}
N^{-2} \mathbf{Y}^{\prime} \mathbf{Y} & N^{-3 / 2} \mathbf{Y}^{\prime} \mathbf{Z}_{p} \\
N^{-3 / 2} \mathbf{Z}_{p}^{\prime} \mathbf{Y} & T^{-1} \mathbf{Z}_{p}^{\prime} \mathbf{Z}_{p}
\end{array}\right]^{-1}\left[\begin{array}{c}
N^{-1} \mathbf{Y}^{\prime} \mathbf{u} \\
N^{-1 / 2} \mathbf{Z}_{p}^{\prime} \mathbf{u}
\end{array}\right]
$$

where $\mathbf{D}_{N}:=\operatorname{diag}\left[(S N)^{-1} \mathbf{I}_{S},(S N)^{-1 / 2} \mathbf{I}_{p}\right]$. Because $N^{-3 / 2} \mathbf{Z}_{p}^{\prime} \mathbf{Y}=o_{p}(1)$ (as implied by $\mathbf{y}_{i}^{\prime} \mathbf{z}_{j}$ being of $O_{p}(N)$, where $\mathbf{y}_{i}$ is $i$-th vector of matrix $\mathbf{Y}$ and $\mathbf{z}_{j}$ is $j$-th vector of matrix $\mathbf{Z}_{p}, i=1, \ldots, S$ and $j=1, \ldots, p)$, the inverse matrix in (S.3) is asymptotically block diagonal. Moreover, $N^{-2} \mathbf{Y}^{\prime} \mathbf{Y}$ weakly converges to an $S \times S$ diagonal matrix (cf. Burridge and Taylor, 2001a, result (v) of Lemma 3.2(a)), and so we can write the so-called normalised bias statistics as

$$
\begin{aligned}
N \hat{\pi}_{j} & =\frac{T^{-1} \mathbf{y}_{j}^{\prime} \mathbf{u}}{T^{-2} \mathbf{y}_{j}^{\prime} \mathbf{y}_{j}}+o_{p}(1)=\frac{T^{-1} \sum_{n=2}^{N} \sum_{s=1-S}^{0} x_{j, S n+s} \varepsilon_{S n+s}}{T^{-2} \sum_{n=2}^{N} \sum_{s=1-S}^{0} x_{j, S n+s}^{2}}+o_{p}(1), j=0, S / 2 \\
N \hat{\pi}_{\alpha, j} & =\frac{T^{-1} \mathbf{y}_{j}^{\alpha \prime} \mathbf{u}}{T^{-2} \mathbf{y}_{j}^{\alpha \prime} \mathbf{y}_{j}^{\alpha}}+o_{p}(1)=\frac{T^{-1} \sum_{n=2}^{N} \sum_{s=1-S}^{0} x_{j, S n+s}^{\alpha} \varepsilon_{S n+s}}{T^{-2} \sum_{n=2}^{N} \sum_{s=1-S}^{0}\left(x_{j, S n+s}^{\alpha}\right)^{2}}+o_{p}(1), j=1, \ldots, S^{*} \\
N \hat{\pi}_{\beta, j} & =\frac{T^{-1} \mathbf{y}_{j}^{\beta \prime} \mathbf{u}}{T^{-2} \mathbf{y}_{j}^{\beta \prime} \mathbf{y}_{j}^{\beta}}+o_{p}(1)=\frac{T^{-1} \sum_{n=2}^{N} \sum_{s=1-S}^{0} x_{j, S n+s}^{\beta} \varepsilon_{S n+s}}{T^{-2} \sum_{n=2}^{N} \sum_{s=1-S}^{0}\left(x_{j, S n+s}^{\beta}\right)^{2}}+o_{p}(1), j=1, \ldots, S^{*} .
\end{aligned}
$$

To find the limiting distribution of the normalised bias statistics, first notice that, using Lemma 1, the CMT and proceeding as in Boswijk et al. (2016, proof of Lemma 1), the following results hold:

$$
\begin{aligned}
N^{-1} \sum_{n=2}^{N} \mathbf{X}_{n-1} \mathcal{E}_{n}^{\prime} & \Rightarrow \mathbf{\Psi}(1) \int_{0}^{1} \mathbf{M}(r) d \mathbf{M}(r)^{\prime}=: \mathbf{Q}_{1} \\
N^{-1} \sum_{n=2}^{N} \mathbf{X}_{n-1} \mathbf{X}_{n-1}^{\prime} & \Rightarrow \mathbf{\Psi}(1) \int_{0}^{1} \mathbf{M}(r) \mathbf{M}(r)^{\prime} d r \mathbf{\Psi}(1)^{\prime}=: \mathbf{Q}_{2} .
\end{aligned}
$$

Consider next the denominators of the normalised bias statistics in (S.4)-(S.6). By standard 
manipulations (see Smith et al., 2009, pp. 557-560)

$$
\begin{aligned}
T^{-2} \sum_{n=2}^{N} \sum_{s=1-S}^{0} x_{j, S n+s}^{2} & =T^{-2} \sum_{n=2}^{N} \mathbf{X}_{j, n}^{\prime} \mathbf{X}_{j, n}=T^{-2} \sum_{n=2}^{N} S\left(\mathbf{X}_{n-1}^{\prime} C_{j} \mathbf{X}_{n-1}\right)+o_{p}(1) \\
& =T^{-2} \sum_{n=2}^{N} S\left(\mathbf{X}_{n-1}^{\prime} c_{j} c_{j}^{\prime} \mathbf{X}_{n-1}\right)+o_{p}(1) \\
& =T^{-2} \sum_{n=2}^{N} \operatorname{tr}\left\{S\left(c_{j}^{\prime} \mathbf{X}_{n-1} \mathbf{X}_{n-1}^{\prime} c_{j}\right)\right\}+o_{p}(1)=S c_{j}^{\prime} \frac{1}{T^{2}} \sum_{n=2}^{N} \mathbf{X}_{n-1} \mathbf{X}_{n-1}^{\prime} c_{j}+o_{p}(1) \\
& \Rightarrow \frac{1}{S} c_{j}^{\prime} \mathbf{\Psi}(1) \int_{0}^{1} \mathbf{M}(r) \mathbf{M}(r)^{\prime} d r \mathbf{\Psi}(1)^{\prime} c_{j}=\frac{1}{S} c_{j}^{\prime} \mathbf{Q}_{2} c_{j}, j=0, S / 2,
\end{aligned}
$$

where "tr" denotes the usual matrix trace operator. Furthermore,

$$
\begin{aligned}
& T^{-2} \sum_{n=2}^{N} \sum_{1-S}^{0}\left(x_{j, S n+s}^{\alpha}\right)^{2}=T^{-2} \sum_{n=2}^{N} \mathbf{X}_{j, n}^{\alpha^{\prime}} \mathbf{X}_{j, n}^{\alpha}=T^{-2} \sum_{n=2}^{N} \frac{S}{2}\left(\mathbf{X}_{n-1}^{\prime} C_{j} \mathbf{X}_{n-1}\right)+o_{p}(1) \\
&=T^{-2} \sum_{n=2}^{N} \frac{S}{2}\left(\mathbf{X}_{n-1}^{\prime} \mathbf{c}_{j} \mathbf{c}_{j}^{\prime} \mathbf{X}_{n-1}\right)+o_{p}(1)=T^{-2} \sum_{n=2}^{N} \frac{S}{2} \operatorname{tr}\left\{\left(\mathbf{c}_{j}^{\prime} \mathbf{X}_{n-1} \mathbf{X}_{\mathrm{n}-1}^{\prime} \mathbf{c}_{\mathbf{j}}\right)\right\}+o_{p}(1) \\
&=T^{-2} \sum_{n=2}^{N} \frac{S}{2} \operatorname{tr}\left(\left[\begin{array}{c}
c_{j}^{\prime} \\
\tilde{c}_{j}^{\prime}
\end{array}\right] \mathbf{X}_{\mathrm{n}-1} \mathbf{X}_{\mathrm{n}-1}^{\prime}\left[\begin{array}{cc}
c_{j} & \tilde{c}_{j}
\end{array}\right]\right)+o_{p}(1) \\
&=T^{-2} \sum_{n=2}^{N} \frac{S}{2} \operatorname{tr}\left[\begin{array}{ll}
c_{j}^{\prime} \mathbf{X}_{n-1} \mathbf{X}_{n-1}^{\prime} c_{j} & \left.c_{j}^{\prime} \mathbf{X}_{n-1} \mathbf{X}_{n-1}^{\prime} \tilde{c}_{j}\right] \\
\mathbf{c}_{n-1} \mathbf{X}_{n-1}^{\prime} c_{j} & \tilde{c}_{j}^{\prime} \mathbf{X}_{n-1} \mathbf{X}_{n-1}^{\prime} \tilde{c}_{j}
\end{array}\right]+o_{p}(1) \\
&= \frac{S}{2} T^{-2} \sum_{n=2}^{N}\left(c_{j}^{\prime} \mathbf{X}_{n-1} \mathbf{X}_{n-1}^{\prime} c_{j}+\tilde{c}_{j}^{\prime} \mathbf{X}_{n-1} \mathbf{X}_{n-1}^{\prime} \tilde{c}_{j}\right)+o_{p}(1) \\
& \Rightarrow \frac{1}{2 S}\left(c_{j}^{\prime} \mathbf{\Psi}(1) \int_{0}^{1} \mathbf{M}(r) \mathbf{M}(r)^{\prime} d r \mathbf{\Psi}(1)^{\prime} c_{j}+\tilde{c}_{j}^{\prime} \mathbf{\Psi}(1) \int_{0}^{1} \mathbf{M}(r) \mathbf{M}(r)^{\prime} d r \mathbf{\Psi}(1)^{\prime} \tilde{c}_{j}\right) \\
&=\frac{1}{2 S}\left[c_{j}^{\prime} \mathbf{Q}_{2} c_{j}+\tilde{c}_{j}^{\prime} \mathbf{Q}_{2} \tilde{c}_{j}\right], j=1, \ldots, S^{*},
\end{aligned}
$$

and

$$
\begin{aligned}
T^{-2} \sum_{n=2}^{N} \sum_{1-S}^{0}\left(x_{j, S n+s}^{\beta}\right)^{2} & =T^{-2} \sum_{n=2}^{N} \mathbf{X}_{j, n}^{\beta^{\prime}} \mathbf{X}_{j, n}^{\beta}=T^{-2} \sum_{n=2}^{N}\left(\mathbf{X}_{n-1}^{\prime} \tilde{C}_{j} \tilde{C}_{j} \mathbf{X}_{n-1}\right)+o_{p}(1) \\
& =T^{-2} \sum_{n=2}^{N} \frac{S}{2}\left(\mathbf{X}_{n-1}^{\prime} C_{j} \mathbf{X}_{n-1}\right)+o_{p}(1) \Rightarrow \frac{1}{2 S}\left[c_{j}^{\prime} \mathbf{Q}_{2} c_{j}+\tilde{c}_{j}^{\prime} \mathbf{Q}_{2} \tilde{c}_{j}\right], j=1, \ldots, S^{*} .
\end{aligned}
$$

Next, consider the numerators of the zero and Nyquist frequency normalised bias statistics. By similar arguments to those used above we obtain that

$$
\begin{aligned}
T^{-1} \sum_{n=2}^{N} \sum_{s=1-S}^{0} x_{j, S n+s} \varepsilon_{S n+s} & =T^{-1} \sum_{n=2}^{N} S\left(\mathbf{X}_{n-1}^{\prime} c_{j} c_{j}^{\prime} \mathcal{E}_{n}\right)+o_{p}(1) \\
& \Rightarrow \frac{1}{S} c_{j}^{\prime} \mathbf{\Psi}(1) \int_{0}^{1} \mathbf{M}(r) d \mathbf{M}(r)^{\prime} c_{j}, j=0, S / 2,
\end{aligned}
$$


while, for the seasonal harmonic frequencies,

$$
\begin{aligned}
T^{-1} \sum_{n=2}^{N} \sum_{s=1-S}^{0} x_{j, S n+s}^{\alpha} \varepsilon_{S n+s} & =T^{-1} \sum_{n=2}^{N} S\left(\mathbf{X}_{n-1}^{\prime} \mathbf{c}_{j} \mathbf{c}_{j}^{\prime} \mathcal{E}_{n}\right)+o_{p}(1) \\
& \Rightarrow \frac{1}{S}\left(c_{j}^{\prime} \mathbf{\Psi}(1) \int_{0}^{1} \mathbf{M}(r) d \mathbf{M}(r)^{\prime} c_{j}+\tilde{c}_{j}^{\prime} \mathbf{\Psi}(1) \int_{0}^{1} \mathbf{M}(r) d \mathbf{M}(r)^{\prime} \tilde{c}_{j}\right) \\
& =\frac{1}{S}\left[c_{j}^{\prime} \mathbf{Q}_{1} c_{j}+\tilde{c}_{j}^{\prime} \mathbf{Q}_{1} \tilde{c}_{j}\right], j=1, \ldots, S^{*},
\end{aligned}
$$

and

$$
\begin{aligned}
T^{-1} \sum_{n=2}^{N} \sum_{s=1-S}^{0} x_{j, S n+s}^{\beta} \varepsilon_{S n+s} & =T^{-1} \sum_{n=2}^{N} S\left(\mathbf{X}_{n-1}^{\prime} \tilde{\mathbf{c}}_{j} \mathbf{c}_{j}^{\prime} \mathcal{E}_{n}\right)+o_{p}(1) \\
& \Rightarrow \frac{1}{2 S}\left(c_{j}^{\prime} \mathbf{\Psi}(1) \int_{0}^{1} \mathbf{M}(r) d \mathbf{M}(r)^{\prime} \tilde{c}_{j}-\tilde{c}_{j}^{\prime} \mathbf{\Psi}(1) \int_{0}^{1} \mathbf{M}(r) d \mathbf{M}(r)^{\prime} c_{j}\right) \\
& =\frac{1}{2 S}\left[c_{j}^{\prime} \mathbf{Q}_{1} \tilde{c}_{j}-\tilde{c}_{j}^{\prime} \mathbf{Q}_{1} c_{j}\right], j=1, \ldots, S^{*} .
\end{aligned}
$$

Finally, by noting, that $\hat{\sigma}^{2}:=T^{-1} \sum_{n=2}^{N} \sum_{s=1-S}^{0}\left(\hat{\varepsilon}_{S n+s}\right)^{2} \rightarrow_{p} \frac{1}{S} c_{0}^{\prime} \boldsymbol{\Sigma}(1) c_{0}$ and that the $\boldsymbol{\Psi}(1)$ matrix drops out of the representations for the limiting distributions of the statistics (this result is demonstrated in the proof of Corollary 1 below), the stated results obtain.

Proof of Corollary 1:

First we define the $S$ (normalised) variance-transformed Brownian motion processes

$$
\begin{aligned}
B_{\eta, i}(\cdot) & :=\left(c_{i}^{\prime} \boldsymbol{\Sigma}(1) c_{i}\right)^{-1 / 2} c_{i}^{\prime} \mathbf{M}(\cdot), i=0, S / 2, \\
B_{\eta, k}(\cdot) & :=\left(c_{k}^{\prime} \boldsymbol{\Sigma}(1) c_{k}\right)^{-1 / 2} c_{k}^{\prime} \mathbf{M}(\cdot), B_{\eta, k}^{*}(\cdot):=\left(\tilde{c}_{k}^{\prime} \boldsymbol{\Sigma}(1) \tilde{c}_{k}\right)^{-1 / 2} \tilde{c}_{k}^{\prime} \mathbf{M}(\cdot), k=1, \ldots, S^{*}
\end{aligned}
$$

which are seen to be mutually independent because of the mutual orthogonality of the selection vectors $c_{i}, i=0, S / 2, c_{k}$ and $\tilde{c}_{k}, k=1, \ldots, S^{*}$. Consider first the $t_{0}$ statistic and write its limiting null distribution as

$$
\begin{aligned}
t_{0} & \Rightarrow \frac{\frac{1}{S} c_{0}^{\prime} \mathbf{Q}_{1} c_{0}}{\sqrt{\frac{1}{S} c_{0}^{\prime} \mathbf{Q}_{2} c_{0} \cdot c_{0}^{\prime} \mathbf{\Sigma}(1) c_{0}}}=\frac{\frac{1}{S} c_{0}^{\prime} \mathbf{\Psi}(1) \mathbf{Q}_{1}^{*} c_{0}}{\sqrt{c_{0}^{\prime} \mathbf{\Psi}(1) \mathbf{Q}_{2}^{*} \mathbf{\Psi}(1)^{\prime} c_{0} \cdot c_{0}^{\prime} \mathbf{\Sigma}(1) c_{0}}}=\frac{\psi(1) c_{0}^{\prime} \mathbf{Q}_{1}^{*} c_{0}}{\sqrt{\psi(1) c_{0}^{\prime} \mathbf{Q}_{2}^{*} c_{0} \psi(1) \cdot c_{0}^{\prime} \mathbf{\Sigma}(1) c_{0}}} \\
& =\frac{c_{0}^{\prime}\left(\int_{0}^{1} \mathbf{M}(r) d \mathbf{M}(r)^{\prime}\right) c_{0}}{\sqrt{c_{0}^{\prime}\left(\int_{0}^{1} \mathbf{M}(r) \mathbf{M}(r)^{\prime} d r\right) c_{0} \cdot c_{0}^{\prime} \boldsymbol{\Sigma}(1) c_{0}}}=\frac{\int_{0}^{1} B_{\eta, 0}(r) d B_{\eta, 0}(r)}{\sqrt{\int_{0}^{1} B_{\eta, 0}^{2}(r) d r}}
\end{aligned}
$$

where we have used the result that $c_{0}^{\prime} \boldsymbol{\Psi}(1)=\psi(1) c_{0}^{\prime}$. The proof for the Nyquist frequency $t_{S / 2}$ statistic is similar except that we use the corresponding result that $c_{S / 2} \mathbf{\Psi}(1)=\psi(-1) c_{S / 2}$ and that $c_{S / 2}^{\prime} \boldsymbol{\Sigma}(1) c_{S / 2}=c_{0}^{\prime} \boldsymbol{\Sigma}(1) c_{0}$. Next, consider the $F_{k}, k=1, \ldots, S^{*}$, statistics. By using the results that $c_{k}^{\prime} \boldsymbol{\Psi}(1)=a_{k} \tilde{c}_{k}^{\prime}+b_{k} c_{k}^{\prime}$ and $\tilde{c}_{k}^{\prime} \boldsymbol{\Psi}(1)=b_{k} \tilde{c}_{k}^{\prime}-a_{k} c_{k}^{\prime}$, where $a_{k}:=\operatorname{I} m\left(\psi\left[\exp \left(i \omega_{k}\right)\right]\right)$ and $b_{k}:=$ $\mathcal{R} e\left(\psi\left[\exp \left(i \omega_{k}\right)\right]\right), k=1, \ldots, S^{*}, \mathcal{R} e(\cdot)$ and $\operatorname{I} m(\cdot)$ denoting the real and imaginary parts of their 
arguments, respectively (see Smith et al., 2009), we have that, for $k=1, \ldots, S^{*}$,

$$
\begin{aligned}
c_{k}^{\prime} \mathbf{Q}_{1} & =\left(b_{k} c_{k}^{\prime}+a_{k} \tilde{c}_{k}^{\prime}\right)\left(\int_{0}^{1} \mathbf{M}(r) d \mathbf{M}(r)^{\prime}\right), \tilde{c}_{k}^{\prime} \mathbf{Q}_{1}=\left(b_{k} \tilde{c}_{k}^{\prime}-a_{k} c_{k}^{\prime}\right)\left(\int_{0}^{1} \mathbf{M}(r) d \mathbf{M}(r)^{\prime}\right) \\
c_{k}^{\prime} \mathbf{Q}_{2} c_{k} & =\left(b_{k} c_{k}^{\prime}+a_{k} \tilde{c}_{k}^{\prime}\right)\left(\int_{0}^{1} \mathbf{M}(r) \mathbf{M}(r)^{\prime} d r\right)\left(b_{k} c_{k}^{\prime}+a_{k} \tilde{c}_{k}^{\prime}\right) \\
\tilde{c}_{k}^{\prime} \mathbf{Q}_{2} \tilde{c}_{k} & =\left(b_{k} \tilde{c}_{k}^{\prime}-a_{k} c_{k}^{\prime}\right)\left(\int_{0}^{1} \mathbf{M}(r) \mathbf{M}(r)^{\prime} d r\right)\left(b_{k} \tilde{c}_{k}^{\prime}-a_{k} c_{k}^{\prime}\right) .
\end{aligned}
$$

Consequently, for each of $k=1, \ldots, S^{*}$, we have that

$$
\begin{aligned}
& \left(c_{k}^{\prime} \mathbf{Q}_{1} c_{k}+\tilde{c}_{k}^{\prime} \mathbf{Q}_{1} \tilde{c}_{k}\right)=\frac{S}{2} a_{k}\left\{c_{k}^{\prime} \boldsymbol{\Sigma}(1) c_{k}\right\}^{1 / 2}\left\{\tilde{c}_{k}^{\prime} \boldsymbol{\Sigma}(1) \tilde{c}_{k}\right\}^{1 / 2}\left(\int_{0}^{1} B_{\eta, k}^{*}(r) d B_{\eta, k}(r)-\int_{0}^{1} B_{\eta, k}(r) d B_{\eta, k}^{*}(r)\right) \\
& +\frac{S}{2} b_{k}\left(\left\{c_{k}^{\prime} \boldsymbol{\Sigma}(1) c_{k}\right\} \int_{0}^{1} B_{\eta, k}(r) d B_{\eta, k}(r)+\left\{\tilde{c}_{k}^{\prime} \boldsymbol{\Sigma}(1) \tilde{c}_{k}\right\} \int_{0}^{1} B_{\eta, k}^{*}(r) d B_{\eta, k}^{*}(r)\right) \\
& \left(c_{k}^{\prime} \mathbf{Q}_{1} \tilde{c}_{k}-\tilde{c}_{k}^{\prime} \mathbf{Q}_{1} c_{k}\right)=\frac{S}{2} b_{k}\left\{c_{k}^{\prime} \boldsymbol{\Sigma}(1) c_{k}\right\}^{1 / 2}\left\{\tilde{c}_{k}^{\prime} \boldsymbol{\Sigma}(1) \tilde{c}_{k}\right\}^{1 / 2}\left(\int_{0}^{1} B_{\eta, k}(r) d B_{\eta, k}^{*}(r)-\int_{0}^{1} B_{\eta, k}^{*}(r) d B_{\eta, k}(r)\right) \\
& +\frac{S}{2} a_{k}\left(\left\{c_{k}^{\prime} \boldsymbol{\Sigma}(1) c_{k}\right\} \int_{0}^{1} B_{\eta, k}(r) d B_{\eta, k}(r)+\left\{\tilde{c}_{k}^{\prime} \boldsymbol{\Sigma}(1) \tilde{c}_{k}\right\} \int_{0}^{1} B_{\eta, k}^{*}(r) d B_{\eta, k}^{*}(r)\right) \\
& \left(c_{k}^{\prime} \mathbf{Q}_{2} c_{k}+\tilde{c}_{k}^{\prime} \mathbf{Q}_{2} \tilde{c}_{k}\right)=\frac{S}{2}\left(a_{k}^{2}+b_{k}^{2}\right)\left(\left\{c_{k}^{\prime} \boldsymbol{\Sigma}(1) c_{k}\right\} \int_{0}^{1} B_{\eta, k}(r)^{2} d r+\left\{\tilde{c}_{k}^{\prime} \boldsymbol{\Sigma}(1) \tilde{c}_{k}\right\} \int_{0}^{1} B_{\eta, k}^{2 *}(r) d r\right) \\
& \text { and, }\left(\frac{1}{S}\left(c_{k}^{\prime} \mathbf{Q}_{1} c_{k}+\tilde{c}_{k}^{\prime} \mathbf{Q}_{1} \tilde{c}_{k}\right)\right)^{2}+\left(\frac{1}{S}\left(c_{k}^{\prime} \mathbf{Q}_{1} \tilde{c}_{k}-\tilde{c}_{k}^{\prime} \mathbf{Q}_{1} c_{k}\right)\right)^{2} \\
& \begin{aligned}
=\frac{a_{k}^{2}+b_{k}^{2}}{S}\left[\left\{c_{k}^{\prime} \boldsymbol{\Sigma}(1) c_{k}\right\}\right. & \left\{\tilde{c}_{k}^{\prime} \boldsymbol{\Sigma}(1) \tilde{c}_{k}\right\}\left(\int_{0}^{1} B_{\eta, k}^{*}(r) d B_{\eta, k}(r)-\int_{0}^{1} B_{\eta, k}(r) d B_{\eta, k}^{*}(r)\right)^{2} \\
& \left.+\left(\left\{c_{k}^{\prime} \boldsymbol{\Sigma}(1) c_{k}\right\} \int_{0}^{1} B_{\eta, k}(r) d B_{\eta, k}(r)+\left\{\tilde{c}_{k}^{\prime} \boldsymbol{\Sigma}(1) \tilde{c}_{k}\right\} \int_{0}^{1} B_{\eta, k}^{*}(r) d B_{\eta, k}^{*}(r)\right)^{2}\right] .
\end{aligned}
\end{aligned}
$$

The stated results for the $F_{k}, k=1, \ldots, S^{*}$, statistics then follow after some routine algebra; notice in particular that that the nuisance parameter term $\left(a_{k}^{2}+b_{k}^{2}\right)$ arising from the weak dependence in $u_{t}$ cancels from these expressions. The results for the joint frequency $F$ statistics then follow directly from the results given above coupled with the asymptotic orthogonality condition; again, see Burridge and Taylor (2001a, result (v) of Lemma 3.2(a)).

Proof of Proposition 2:

The following proof holds regardless of whether Step 2a or Step 2b is used in Algorithm 1.

Let $S_{N}^{b}(r):=N^{-1 / 2} \mathbf{X}_{\lfloor r N\rfloor}^{*}$. Under Assumption 1 we have by Boswijk et al. (2016), Lemma 4, that

$$
S_{N}^{b}(r)=N^{-1 / 2} \sum_{n=2}^{\lfloor r N\rfloor} \mathbf{E}_{n}^{*} \Rightarrow_{p} \mathbf{M}(r)
$$

where $\mathbf{E}_{n}^{*}=\left[\varepsilon_{S n-(S-1)}^{*}, \varepsilon_{S n-(S-2)}^{*}, \ldots, \varepsilon_{S n}^{*}\right]^{\prime}$, because, conditionally on $\left\{\hat{\mathbf{E}}_{n}\right\}_{n=2}^{N}, S_{N}^{b}(\cdot)$ is a Gaussian process with independent increments and covariance kernel $E^{*}\left(S_{N}^{b}(\cdot) S_{N}^{b}(\cdot)^{\prime-1} \sum_{n=2}^{\lfloor\cdot N\rfloor} \hat{\mathbf{E}}_{n} \hat{\mathbf{E}}_{n}^{\prime}\right.$ (here $E^{*}$ 
denotes expectation under bootstrap probability measure $\left.P^{*}\right)$, where $N^{-1} \sum_{n=2}^{\lfloor r N\rfloor} \hat{\mathbf{E}}_{n} \hat{\mathbf{E}}_{n}^{\prime} \rightarrow \boldsymbol{\Sigma}(r)$ in probability uniformly for all $u \in[0,1]$; see Boswijk et al. (2016), Lemma 4. By the same Lemma,

$$
N^{-1} \sum_{n=2}^{N} \mathbf{X}_{n-1}^{*} \mathbf{E}_{n}^{*^{\prime}} \Rightarrow_{p} \int_{0}^{1} \mathbf{M}(r) d \mathbf{M}(r)^{\prime}=: \mathbf{Q}_{1}^{*} \text { and } N^{-1} \sum_{n=2}^{N} \mathbf{X}_{n-1}^{*} \mathbf{X}_{n-1}^{*^{\prime}} \Rightarrow_{p} \int_{0}^{1} \mathbf{M}(r) \mathbf{M}(r)^{\prime} d r=: \mathbf{Q}_{2}^{*}
$$

jointly with (S.13). Therefore, as in the proof of (S.7)-(S.12), we obtain

$$
\begin{gathered}
T^{-2} \sum_{n=2}^{N} \sum_{s=1-S}^{0} x_{j, S n+s}^{* 2} \Rightarrow_{p} \frac{1}{S} c_{j}^{\prime} \int_{0}^{1} \mathbf{M}(r) \mathbf{M}(r)^{\prime} d r c_{j}, j=0, S / 2, \\
T^{-2} \sum_{n=2}^{N} \sum_{1-S}^{0}\left(x_{j, S n+s}^{* \alpha}\right)^{2} \Rightarrow_{p} \frac{1}{2 S}\left[c_{j}^{\prime} \mathbf{Q}_{2}^{*} c_{j}+\tilde{c}_{j}^{\prime} \mathbf{Q}_{2}^{*} \tilde{c}_{j}\right], j=1, \ldots, S^{*}, \\
T^{-2} \sum_{n=2}^{N} \sum_{1-S}^{0}\left(x_{j, S n+s}^{* \beta}\right)^{2} \Rightarrow_{p} \frac{1}{2 S}\left[c_{j}^{\prime} \mathbf{Q}_{2}^{*} c_{j}+\tilde{c}_{j}^{\prime} \mathbf{Q}_{2}^{*} \tilde{c}_{j}\right], j=1, \ldots, S^{*}, \\
T^{-1} \sum_{n=2}^{N} \sum_{s=1-S}^{0} x_{j, S n+s}^{*} \varepsilon_{S n+s}^{*} \Rightarrow_{p} \frac{1}{S} c_{j}^{\prime} \int_{0}^{1} \mathbf{M}(r) d \mathbf{M}(r)^{\prime} c_{j}, j=0, S / 2 \\
T^{-1} \sum_{n=2}^{N} \sum_{s=1-S}^{0} x_{j, S n+s}^{* \alpha} \varepsilon_{S n+s}^{*} \Rightarrow_{p} \frac{1}{2 S}\left[c_{j}^{\prime} \mathbf{Q}_{1}^{*} c_{j}+\tilde{c}_{j}^{\prime} \mathbf{Q}_{1}^{*} \tilde{c}_{j}\right], j=1, \ldots, S^{*}, \\
T^{-1} \sum_{n=2}^{N} \sum_{s=1-S}^{0} x_{j, S n+s}^{* \beta} \varepsilon_{S n+s}^{*} \Rightarrow_{p} \frac{1}{2 S}\left[c_{j}^{\prime} \mathbf{Q}_{1}^{*} \tilde{c}_{j}-\tilde{c}_{j}^{\prime} \mathbf{Q}_{1}^{*} c_{j}\right], j=1, \ldots, S^{*} .
\end{gathered}
$$

Because the (asymptotic) orthogonality results also hold for the bootstrap series, all that remains is to establish the limiting behaviour of $\hat{\sigma}^{* 2}:=T^{-1} \sum_{n=2}^{N} \sum_{s=1-S}^{0}\left(\hat{\varepsilon}_{S n+s}^{*}\right)^{2}$. To that end, observe first that under the conditions of Proposition 2, $\hat{\sigma}^{* 2}=T^{-1} \sum_{n=2}^{N} \sum_{s=1-S}^{0}\left(\Delta_{S} x_{S n+s}^{*}\right)^{2}+o_{p}^{*}(1)=$ $T^{-1} \sum_{n=2}^{N} \sum_{s=1-S}^{0}\left(\varepsilon_{S n+s}^{*}\right)^{2}+o_{p}^{*}(1)$. Now $T^{-1} \sum_{n=2}^{N} \sum_{s=1-S}^{0}\left(\varepsilon_{S n+s}^{*}\right)^{2}=T^{-1} \sum_{n=2}^{N} \sum_{s=1-S}^{0} \hat{\varepsilon}_{S n+s}^{2} w_{n}^{2}$ $=T^{-1} \sum_{n=2}^{N} \sum_{s=1-S}^{0} \hat{\varepsilon}_{S n+s}^{2}+T^{-1} \sum_{n=2}^{N} \sum_{s=1-S}^{0} \hat{\varepsilon}_{S n+s}^{2} \xi_{n}=: f_{1}+f_{2}$, where $\xi_{n}:=w_{n}^{2}-1$ is an independent sequence of centered $\chi^{2}(1)$ random variables, and $f_{1}$ and $f_{2}$ are implicitly defined. The first term, $f_{1}$, converges to $\frac{1}{S} c_{0}^{\prime} \boldsymbol{\Sigma}(1) c_{0}$, while, conditionally on the original sample, $E^{*}\left(f_{2}^{2}\right)$ is of $O_{p}\left(T^{-1}\right)$. The latter because $E^{*}\left(T^{-1} \sum_{n=2}^{N} \sum_{s=1-S}^{0} \hat{\varepsilon}_{S n+s}^{2} \xi_{n}\right)^{2}=T^{-2} E^{*}\left(\sum_{\tilde{n}=1}^{N} \sum_{\tilde{s}=1-S}^{0} \sum_{n=2}^{N} \sum_{s=1-S}^{0} \hat{\varepsilon}_{S n+s}^{2} \hat{\varepsilon}_{S \tilde{n}+\tilde{s}}^{2} \xi_{n} \xi_{\tilde{n}}\right)$ $=T^{-2} E^{*}\left(\sum_{n=2}^{N} \sum_{s=1-S}^{0} \hat{\varepsilon}_{S n+s}^{4} E\left(\xi_{n}^{2}\right)\right)^{2}=4 T^{-1}\left(T^{-1} \sum_{n=2}^{N} \sum_{s=1-S}^{0} \hat{\varepsilon}_{S n+s}^{4}\right)^{2}=O_{p}\left(T^{-1}\right)$, by virtue of the fact that $T^{-1} \sum_{n=2}^{N} \sum_{s=1-S}^{0} \varepsilon_{S n+s}^{4}$ is of $O_{p}(1)$ under Assumption 1. Consequently, $\hat{\sigma}^{* 2} \stackrel{p^{*}}{\rightarrow}$ $\frac{1}{S} c_{0}^{\prime} \boldsymbol{\Sigma}(1) c_{0}$.

The results stated in the first part of the proposition regarding the first-order limiting distributions of the bootstrap HEGY statistics then follow straightforwardly. Turning to the second part of the proposition, consider first the bootstrap $t_{0}^{*}$ statistic. The result that $t_{0}^{*} \Rightarrow_{p} \xi_{\eta, 0}$ implies that, uniformly in probability, $G_{0, T}^{*}(\cdot) \rightarrow G_{0}(\cdot)$, where $G_{0}(\cdot)$ denotes the cdf of $\xi_{\eta, 0}$. As with the proof of Corollary 1 of Hansen (2000), establishing that $P_{T}^{*}$ converges weakly to $U[0,1]$ under the conditions of the proposition is then entirely straightforward and, hence, is omitted in the interests of brevity. The corresponding results for the other HEGY statistics follow in similar fashion. 


\section{S.3 Additional Monte Carlo Results}

This section contains additional Monte Carlo results relating to the wild bootstrap HEGY tests which use the seasonal block wild re-sampling scheme outlined in Step 2b of Algorithm 1. Tables S.1-S.6 give complementary results to those given in Tables 1-6 respectively. The Monte Carlo DGP and set-up of these experiments were otherwise exactly as detailed in Section 5. 
Table S.1. Empirical sizes of conventional and wild bootstrap HEGY tests using Step 2b of Algorithm 1. OLS de-trending.

\begin{tabular}{|c|c|c|c|c|c|c|c|c|c|c|c|c|c|}
\hline$\Sigma$ & $\delta$ & $\tau$ & $N$ & $t_{0}$ & $t_{2}$ & $F_{1}$ & $F_{12}$ & $F_{012}$ & $t_{0}^{*}$ & $t_{2}^{*}$ & $F_{1}^{*}$ & $F_{12}^{*}$ & $F_{012}^{*}$ \\
\hline \multicolumn{14}{|c|}{ Model 1: Homoskedasticity } \\
\hline & & & 50 & 3.1 & 4.6 & 3.7 & 4.6 & 4.4 & 4.6 & 5.3 & 4.1 & 4.0 & 3.9 \\
\hline & & & 100 & 3.9 & 4.7 & 4.2 & 4.6 & 4.7 & 5.0 & 5.2 & 4.2 & 4.3 & 4.3 \\
\hline \multicolumn{14}{|c|}{ Model 2: Periodic heteroskedasticity } \\
\hline \multirow[t]{2}{*}{ Case 1} & & & 50 & 4.0 & 4.7 & 6.0 & 6.3 & 6.6 & 6.2 & 5.5 & 5.4 & 4.9 & 4.8 \\
\hline & & & 100 & 4.5 & 5.1 & 6.5 & 6.4 & 6.9 & 5.9 & 5.3 & 5.3 & 5.0 & 5.0 \\
\hline Case 2 & & & 50 & 3.7 & 4.4 & 9.1 & 13.2 & 13.0 & 7.8 & 5.7 & 5.7 & 5.7 & 5.8 \\
\hline & & & 100 & 4.2 & 4.9 & 9.4 & 13.3 & 13.0 & 6.6 & 5.3 & 5.5 & 5.3 & 5.4 \\
\hline \multicolumn{14}{|c|}{ Model 3: Single volatility shift } \\
\hline & 0.33 & 0.2 & 50 & 1.9 & 2.1 & 1.4 & 1.4 & 1.4 & 4.1 & 4.6 & 3.6 & 3.5 & 3.5 \\
\hline & & & 100 & 2.6 & 2.3 & 1.6 & 1.5 & 1.3 & 4.7 & 4.8 & 4.0 & 4.1 & 4.0 \\
\hline & & 0.8 & 50 & 5.0 & 5.0 & 6.5 & 6.9 & 6.4 & 5.3 & 5.2 & 4.6 & 4.4 & 4.2 \\
\hline & & & 100 & 6.1 & 5.9 & 7.6 & 7.9 & 7.4 & 5.2 & 5.0 & 5.2 & 4.5 & 4.4 \\
\hline & 3 & 0.2 & 50 & 20.1 & 27.5 & 42.6 & 54.6 & 61.5 & 9.8 & 10.5 & 9.8 & 9.8 & 9.0 \\
\hline & & & 100 & 21.6 & 29.0 & 44.4 & 56.8 & 62.3 & 7.2 & 7.9 & 7.8 & 7.5 & 6.8 \\
\hline & & 0.8 & 50 & 4.7 & 6.3 & 6.6 & 7.7 & 8.4 & 5.1 & 5.5 & 4.8 & 4.1 & 4.0 \\
\hline & & & 100 & 5.6 & 6.4 & 6.8 & 7.7 & 8.6 & 4.9 & 4.9 & 4.5 & 4.0 & 4.1 \\
\hline \multicolumn{14}{|c|}{ Model 4: Single periodic volatility shift } \\
\hline \multirow[t]{8}{*}{ Case 1} & 0.33 & 0.2 & 50 & 2.5 & 2.1 & 1.8 & 1.6 & 1.8 & 5.7 & 4.4 & 3.9 & 3.6 & 3.9 \\
\hline & & & 100 & 2.8 & 2.3 & 2.2 & 1.6 & 1.9 & 5.1 & 4.7 & 4.2 & 4.1 & 4.1 \\
\hline & & 0.8 & 50 & 5.4 & 5.3 & 6.9 & 7.0 & 7.7 & 6.1 & 5.0 & 5.1 & 4.8 & 4.9 \\
\hline & & & 100 & 6.7 & 6.1 & 7.8 & 7.8 & 8.3 & 6.0 & 5.2 & 4.8 & 4.5 & 4.7 \\
\hline & 3 & 0.2 & 50 & 19.8 & 27.5 & 40.0 & 51.5 & 56.9 & 9.7 & 10.2 & 10.4 & 9.7 & 9.4 \\
\hline & & & 100 & 21.5 & 28.6 & 40.7 & 53.3 & 57.8 & 7.9 & 7.9 & 8.1 & 7.4 & 7.5 \\
\hline & & 0.8 & 50 & 4.9 & 6.5 & 8.3 & 9.2 & 10.4 & 5.8 & 5.5 & 5.2 & 4.8 & 4.7 \\
\hline & & & 100 & 5.7 & 6.8 & 8.6 & 9.2 & 10.7 & 5.6 & 5.2 & 4.9 & 4.8 & 4.8 \\
\hline \multirow[t]{8}{*}{ Case 2} & 0.33 & 0.2 & 50 & 2.5 & 2.3 & 4.6 & 6.5 & 6.9 & 7.7 & 4.7 & 4.6 & 4.8 & 4.9 \\
\hline & & & 100 & 2.8 & 2.4 & 4.4 & 6.5 & 6.8 & 6.5 & 4.6 & 4.6 & 4.6 & 4.8 \\
\hline & & 0.8 & 50 & 5.8 & 6.3 & 10.8 & 14.2 & 14.6 & 8.8 & 6.1 & 6.1 & 6.2 & 6.6 \\
\hline & & & 100 & 6.2 & 6.0 & 11.0 & 14.6 & 15.1 & 6.9 & 5.3 & 5.8 & 5.7 & 5.6 \\
\hline & 3 & 0.2 & 50 & 18.3 & 27.1 & 38.1 & 44.8 & 47.4 & 11.7 & 11.4 & 11.5 & 11.5 & 12.1 \\
\hline & & & 100 & 20.7 & 27.9 & 38.3 & 45.0 & 47.3 & 8.4 & 8.0 & 8.0 & 8.0 & 8.2 \\
\hline & & 0.8 & 50 & 5.0 & 6.3 & 12.0 & 16.5 & 16.6 & 8.2 & 6.0 & 6.2 & 6.3 & 6.4 \\
\hline & & & 100 & 6.0 & 7.6 & 12.7 & 17.5 & 17.5 & 7.1 & 6.0 & 5.9 & 6.0 & 5.9 \\
\hline \multirow[t]{8}{*}{ Case 3} & 0.33 & 0.2 & 50 & 2.4 & 2.1 & 2.2 & 1.8 & 2.2 & 4.2 & 4.7 & 4.0 & 3.9 & 4.1 \\
\hline & & & 100 & 2.9 & 2.2 & 2.5 & 2.2 & 2.3 & 4.4 & 4.5 & 4.3 & 4.1 & 4.0 \\
\hline & & 0.8 & 50 & 3.7 & 4.4 & 4.2 & 4.4 & 4.7 & 4.6 & 5.3 & 4.5 & 4.3 & 4.0 \\
\hline & & & 100 & 4.6 & 4.9 & 4.7 & 4.8 & 5.0 & 4.9 & 4.9 & 4.7 & 4.2 & 4.5 \\
\hline & 3 & 0.2 & 50 & 5.7 & 7.4 & 10.5 & 11.5 & 12.4 & 6.5 & 6.2 & 5.7 & 5.0 & 4.6 \\
\hline & & & 100 & 5.8 & 8.2 & 11.3 & 12.4 & 12.5 & 5.3 & 5.6 & 5.2 & 4.9 & 4.7 \\
\hline & & 0.8 & 50 & 3.7 & 5.0 & 5.0 & 5.4 & 5.8 & 5.1 & 5.6 & 4.6 & 4.1 & 3.9 \\
\hline & & & 100 & 4.4 & 5.3 & 5.3 & 5.7 & 5.9 & 5.0 & 4.9 & 4.6 & 3.9 & 4.1 \\
\hline \multirow[t]{4}{*}{ Case 4} & & 0.2 & 50 & 3.4 & 4.5 & 6.0 & 6.1 & 6.8 & 4.3 & 5.1 & 4.9 & 4.5 & 4.0 \\
\hline & & & 100 & 4.0 & 4.7 & 6.3 & 6.1 & 6.6 & 4.7 & 5.1 & 5.1 & 4.9 & 4.4 \\
\hline & & 0.8 & 50 & 3.8 & 4.2 & 5.3 & 5.4 & 6.1 & 5.2 & 5.6 & 5.1 & 4.4 & 4.2 \\
\hline & & & 100 & 4.0 & 4.6 & 5.7 & 5.8 & 6.2 & 5.3 & 5.5 & 5.2 & 4.9 & 4.8 \\
\hline
\end{tabular}


Table S.2. Empirical sizes of conventional and wild bootstrap HEGY tests using Step 2b of Algorithm 1. Local GLS de-trending.

\begin{tabular}{|c|c|c|c|c|c|c|c|c|c|c|c|c|c|}
\hline$\Sigma$ & $\delta$ & $\tau$ & $N$ & $t_{0}$ & $t_{2}$ & $F_{1}$ & $F_{12}$ & $F_{012}$ & $t_{0}^{*}$ & $t_{2}^{*}$ & $F_{1}^{*}$ & $F_{12}^{*}$ & $F_{012}^{*}$ \\
\hline \multicolumn{14}{|c|}{ Model 1: Homoskedasticity } \\
\hline & & & 50 & 5.9 & 8.7 & 6.6 & 8.1 & 8.7 & 4.8 & 4.9 & 4.6 & 4.5 & 4.3 \\
\hline & & & 100 & 5.2 & 7.2 & 5.8 & 5.8 & 6.7 & 4.6 & 5.1 & 4.8 & 4.4 & 4.8 \\
\hline \multicolumn{14}{|c|}{ Model 2: Periodic heteroskedasticity } \\
\hline \multirow[t]{2}{*}{ Case 1} & & & 50 & 6.5 & 9.6 & 6.5 & 7.5 & 9.6 & 5.8 & 5.4 & 4.7 & 4.6 & 4.8 \\
\hline & & & 100 & 6.1 & 7.1 & 5.7 & 6.3 & 7.9 & 5.9 & 4.9 & 4.7 & 4.7 & 5.1 \\
\hline Case 2 & & & 50 & 6.1 & 9.1 & 10.1 & 13.3 & 13.6 & 8.4 & 5.1 & 5.3 & 5.2 & 5.7 \\
\hline & & & 100 & 5.2 & 7.5 & 9.7 & 12.5 & 12.2 & 6.4 & 5.1 & 5.2 & 5.3 & 5.4 \\
\hline \multicolumn{14}{|c|}{ Model 3: Single volatility shift } \\
\hline & 0.33 & 0.2 & 50 & 6.7 & 10.1 & 9.3 & 10.9 & 11.4 & 4.6 & 5.1 & 4.6 & 4.7 & 4.2 \\
\hline & & & 100 & 6.3 & 8.7 & 9.0 & 9.5 & 9.9 & 4.4 & 4.9 & 4.7 & 4.6 & 4.4 \\
\hline & & 0.8 & 50 & 10.2 & 14.6 & 19.7 & 24.5 & 24.2 & 5.4 & 5.7 & 6.3 & 6.0 & 5.8 \\
\hline & & & 100 & 10.7 & 12.6 & 19.7 & 23.7 & 23.3 & 5.5 & 5.4 & 5.4 & 5.5 & 5.5 \\
\hline & 3 & 0.2 & 50 & 11.6 & 13.5 & 6.6 & 9.9 & 14.0 & 6.1 & 5.8 & 5.6 & 5.6 & 5.5 \\
\hline & & & 100 & 9.2 & 10.2 & 5.7 & 7.4 & 9.6 & 5.4 & 5.4 & 5.3 & 5.3 & 5.2 \\
\hline & & 0.8 & 50 & 6.7 & 9.3 & 5.6 & 6.9 & 9.0 & 4.7 & 4.6 & 4.3 & 4.2 & 4.2 \\
\hline & & & 100 & 6.1 & 7.3 & 4.7 & 5.2 & 6.2 & 4.7 & 4.8 & 4.4 & 4.1 & 4.2 \\
\hline \multicolumn{14}{|c|}{ Model 4: Single periodic volatility shift } \\
\hline \multirow[t]{8}{*}{ Case 1} & 0.33 & 0.2 & 50 & 7.3 & 10.5 & 9.3 & 10.6 & 12.3 & 5.9 & 5.0 & 5.1 & 4.7 & 4.8 \\
\hline & & & 100 & 6.8 & 9.0 & 8.4 & 9.5 & 10.7 & 5.3 & 5.0 & 5.1 & 4.8 & 4.7 \\
\hline & & 0.8 & 50 & 10.8 & 14.8 & 17.7 & 21.6 & 23.5 & 6.5 & 5.7 & 6.0 & 5.6 & 5.4 \\
\hline & & & 100 & 10.8 & 12.6 & 17.2 & 21.2 & 22.5 & 6.1 & 5.6 & 5.5 & 5.6 & 5.4 \\
\hline & 3 & 0.2 & 50 & 11.4 & 12.9 & 7.1 & 10.0 & 15.2 & 7.0 & 5.9 & 5.4 & 5.5 & 6.1 \\
\hline & & & 100 & 9.4 & 9.8 & 6.0 & 7.3 & 10.7 & 5.7 & 5.2 & 5.1 & 4.8 & 4.8 \\
\hline & & 0.8 & 50 & 7.2 & 9.6 & 5.9 & 6.9 & 10.0 & 5.7 & 4.6 & 4.8 & 4.3 & 4.5 \\
\hline & & & 100 & 6.7 & 7.3 & 4.7 & 5.3 & 7.4 & 5.9 & 4.8 & 4.5 & 4.4 & 4.8 \\
\hline \multirow[t]{8}{*}{ Case 2} & 0.33 & 0.2 & 50 & 7.0 & 10.5 & 12.8 & 16.1 & 16.1 & 8.1 & 5.1 & 5.4 & 5.3 & 5.6 \\
\hline & & & 100 & 6.8 & 8.6 & 11.9 & 14.9 & 14.8 & 6.7 & 4.6 & 4.7 & 4.6 & 4.8 \\
\hline & & 0.8 & 50 & 11.2 & 15.4 & 20.2 & 24.3 & 24.4 & 9.1 & 6.1 & 7.1 & 6.9 & 7.0 \\
\hline & & & 100 & 10.5 & 12.7 & 20.1 & 23.7 & 23.2 & 7.3 & 5.3 & 6.4 & 6.4 & 6.2 \\
\hline & 3 & 0.2 & 50 & 11.2 & 13.3 & 12.0 & 15.7 & 19.0 & 10.3 & 6.5 & 6.3 & 6.4 & 7.1 \\
\hline & & & 100 & 8.8 & 9.8 & 10.1 & 13.7 & 15.2 & 7.3 & 5.3 & 5.2 & 5.1 & 5.6 \\
\hline & & 0.8 & 50 & 7.0 & 10.2 & 9.9 & 13.7 & 14.5 & 8.2 & 5.5 & 5.4 & 5.4 & 5.4 \\
\hline & & & 100 & 6.6 & 8.1 & 9.1 & 12.0 & 12.5 & 7.1 & 5.3 & 4.8 & 4.9 & 5.3 \\
\hline \multirow[t]{8}{*}{ Case 3} & 0.33 & 0.2 & 50 & 7.0 & 10.0 & 7.7 & 9.8 & 11.7 & 4.4 & 5.1 & 4.9 & 4.7 & 4.5 \\
\hline & & & 100 & 6.6 & 8.5 & 7.5 & 8.1 & 9.5 & 4.6 & 4.8 & 4.7 & 4.7 & 4.7 \\
\hline & & 0.8 & 50 & 8.0 & 11.8 & 12.5 & 15.3 & 16.5 & 4.7 & 5.4 & 5.5 & 5.5 & 4.9 \\
\hline & & & 100 & 7.9 & 9.2 & 12.5 & 14.2 & 14.5 & 5.0 & 4.9 & 5.2 & 5.4 & 5.0 \\
\hline & 3 & 0.2 & 50 & 6.4 & 8.7 & 4.9 & 6.3 & 8.4 & 5.5 & 4.7 & 4.5 & 4.4 & 4.2 \\
\hline & & & 100 & 5.5 & 7.1 & 4.6 & 5.1 & 6.2 & 5.2 & 5.0 & 4.6 & 4.5 & 4.4 \\
\hline & & 0.8 & 50 & 6.2 & 8.8 & 5.5 & 6.5 & 8.2 & 4.8 & 5.0 & 3.9 & 3.8 & 4.1 \\
\hline & & & 100 & 5.5 & 6.9 & 5.1 & 5.3 & 6.2 & 4.7 & 4.8 & 4.6 & 4.7 & 4.7 \\
\hline \multirow[t]{4}{*}{ Case 4} & & 0.2 & 50 & 5.8 & 8.8 & 6.1 & 7.3 & 9.0 & 4.0 & 4.7 & 4.8 & 4.2 & 4.0 \\
\hline & & & 100 & 5.5 & 6.8 & 5.3 & 5.7 & 6.8 & 4.4 & 5.1 & 4.8 & 4.9 & 4.5 \\
\hline & & 0.8 & 50 & 6.4 & 8.5 & 6.1 & 7.1 & 9.5 & 5.1 & 4.7 & 4.4 & 4.3 & 4.2 \\
\hline & & & 100 & 5.6 & 7.7 & 5.7 & 5.9 & 7.0 & 5.3 & 5.0 & 4.7 & 4.5 & 4.6 \\
\hline
\end{tabular}


Table S.3. Empirical size of conventional and wild bootstrap HEGY tests using Step 2b of Algorithm 1. OLS de-trending. Weakly dependent shocks.

\begin{tabular}{|c|c|c|c|c|c|c|c|c|c|c|c|c|}
\hline & $\Phi / \Theta$ & $N$ & $t_{0}$ & $t_{2}$ & $F_{1}$ & $F_{12}$ & $F_{012}$ & $t_{0}^{*}$ & $t_{2}^{*}$ & $F_{1}^{*}$ & $F_{12}^{*}$ & $F_{012}^{*}$ \\
\hline \multicolumn{13}{|c|}{ Homoskedasticity } \\
\hline \multirow[t]{8}{*}{$\operatorname{AR}(4)$} & -0.8 & 50 & 1.7 & 3.5 & 3.1 & 3.5 & 3.4 & 3.9 & 5.0 & 4.8 & 4.6 & 4.4 \\
\hline & & 100 & 3.1 & 4.4 & 3.9 & 4.2 & 4.0 & 4.3 & 5.1 & 4.8 & 4.8 & 4.7 \\
\hline & -0.5 & 50 & 2.5 & 3.7 & 3.5 & 3.8 & 3.5 & 3.9 & 5.3 & 4.8 & 4.7 & 4.4 \\
\hline & & 100 & 3.5 & 4.1 & 4.0 & 4.3 & 3.9 & 4.6 & 5.3 & 4.7 & 5.0 & 4.6 \\
\hline & 0.5 & 50 & 1.9 & 3.6 & 3.7 & 3.5 & 2.7 & 2.6 & 4.6 & 4.6 & 4.0 & 3.2 \\
\hline & & 100 & 3.6 & 4.4 & 4.3 & 4.5 & 4.3 & 4.9 & 5.2 & 4.8 & 4.6 & 4.4 \\
\hline & 0.8 & 50 & 3.5 & 4.1 & 3.5 & 3.5 & 3.8 & 5.3 & 5.6 & 4.8 & 4.2 & 4.4 \\
\hline & & 100 & 3.7 & 4.6 & 3.7 & 4.0 & 4.0 & 4.6 & 5.2 & 4.3 & 4.3 & 4.3 \\
\hline \multirow[t]{4}{*}{$\operatorname{MA}(2)$} & -0.5 & 50 & 6.7 & 7.6 & 3.2 & 5.4 & 8.3 & 9.9 & 9.1 & 5.1 & 6.9 & 9.2 \\
\hline & & 100 & 6.5 & 6.7 & 3.7 & 5.4 & 6.5 & 8.3 & 7.7 & 4.7 & 5.9 & 7.5 \\
\hline & 0.5 & 50 & 2.8 & 3.7 & 6.2 & 6.2 & 6.0 & 4.0 & 5.2 & 8.2 & 7.2 & 6.3 \\
\hline & & 100 & 2.6 & 4.0 & 5.9 & 5.2 & 4.5 & 3.8 & 4.9 & 6.7 & 5.9 & 5.4 \\
\hline \multicolumn{13}{|c|}{ Periodic heteroskedasticity, Case 2} \\
\hline \multirow[t]{8}{*}{$\operatorname{AR}(4)$} & -0.8 & 50 & 2.9 & 4.1 & 8.1 & 11.6 & 11.4 & 4.6 & 5.6 & 5.6 & 5.6 & 5.7 \\
\hline & & 100 & 3.9 & 4.5 & 9.0 & 12.8 & 12.6 & 4.4 & 5.3 & 5.2 & 5.4 & 5.1 \\
\hline & -0.5 & 50 & 3.3 & 4.4 & 8.8 & 12.5 & 12.5 & 5.6 & 5.7 & 5.8 & 5.9 & 5.8 \\
\hline & & 100 & 4.2 & 4.9 & 9.3 & 12.9 & 13.1 & 5.0 & 5.1 & 5.3 & 5.2 & 5.4 \\
\hline & 0.5 & 50 & 3.0 & 3.9 & 7.8 & 11.1 & 10.8 & 4.3 & 5.0 & 5.1 & 5.0 & 4.9 \\
\hline & & 100 & 3.9 & 4.7 & 9.1 & 13.0 & 13.0 & 5.7 & 5.4 & 5.4 & 5.3 & 5.4 \\
\hline & 0.8 & 50 & 3.8 & 4.5 & 8.7 & 12.6 & 12.2 & 5.7 & 6.2 & 6.2 & 6.1 & 5.9 \\
\hline & & 100 & 4.2 & 4.9 & 9.0 & 12.9 & 12.6 & 5.3 & 5.5 & 5.5 & 5.5 & 5.5 \\
\hline \multirow[t]{4}{*}{$\mathrm{MA}(2)$} & -0.5 & 50 & 4.6 & 4.9 & 7.1 & 11.8 & 12.2 & 7.5 & 7.0 & 5.5 & 5.9 & 6.0 \\
\hline & & 100 & 4.3 & 5.3 & 8.3 & 12.8 & 13.2 & 6.4 & 6.0 & 5.3 & 5.5 & 5.4 \\
\hline & 0.5 & 50 & 2.5 & 3.6 & 10.2 & 13.3 & 12.9 & 4.7 & 5.9 & 7.8 & 7.1 & 6.7 \\
\hline & & 100 & 3.0 & 3.7 & 10.0 & 13.1 & 12.5 & 4.9 & 5.0 & 6.2 & 5.7 & 5.4 \\
\hline \multicolumn{13}{|c|}{ Single volatility shift, $\delta=3, \tau=0.8$} \\
\hline \multirow[t]{8}{*}{$\operatorname{AR}(4)$} & -0.8 & 50 & 2.6 & 5.2 & 5.2 & 6.3 & 6.2 & 4.0 & 5.6 & 5.6 & 4.9 & 4.7 \\
\hline & & 100 & 4.1 & 6.0 & 6.2 & 7.3 & 7.2 & 4.2 & 5.1 & 4.9 & 4.7 & 4.3 \\
\hline & -0.5 & 50 & 3.3 & 5.4 & 5.5 & 6.5 & 6.4 & 4.4 & 5.9 & 5.6 & 5.3 & 4.8 \\
\hline & & 100 & 4.7 & 6.4 & 6.4 & 7.5 & 7.6 & 4.4 & 5.5 & 5.0 & 5.1 & 4.5 \\
\hline & 0.5 & 50 & 2.8 & 4.8 & 5.0 & 5.7 & 5.6 & 3.9 & 5.1 & 4.6 & 4.2 & 3.7 \\
\hline & & 100 & 4.8 & 5.9 & 6.4 & 7.2 & 7.7 & 4.7 & 5.1 & 4.7 & 4.7 & 4.3 \\
\hline & 0.8 & 50 & 4.2 & 5.1 & 4.6 & 5.0 & 5.2 & 5.0 & 5.4 & 4.3 & 4.0 & 4.0 \\
\hline & & 100 & 4.8 & 5.7 & 5.3 & 6.2 & 6.5 & 5.0 & 5.0 & 4.6 & 4.3 & 4.1 \\
\hline \multirow[t]{4}{*}{$\mathrm{MA}(2)$} & -0.5 & 50 & 8.1 & 9.3 & 5.3 & 8.5 & 12.4 & 9.9 & 9.4 & 4.8 & 6.6 & 8.8 \\
\hline & & 100 & 7.3 & 8.4 & 5.7 & 8.4 & 11.0 & 8.2 & 7.4 & 4.8 & 5.6 & 7.0 \\
\hline & 0.5 & 50 & 3.3 & 5.3 & 8.3 & 8.7 & 8.8 & 4.9 & 5.6 & 8.3 & 7.3 & 6.4 \\
\hline & & 100 & 3.7 & 5.5 & 8.2 & 8.7 & 8.2 & 4.3 & 4.8 & 6.2 & 6.0 & 5.1 \\
\hline
\end{tabular}


Table S.3. Continued.

\begin{tabular}{|c|c|c|c|c|c|c|c|c|c|c|c|c|}
\hline & $\Phi / \Theta$ & $N$ & $t_{0}$ & $t_{2}$ & $F_{1}$ & $F_{12}$ & $F_{012}$ & $t_{0}^{*}$ & $t_{2}^{*}$ & $F_{1}^{*}$ & $F_{12}^{*}$ & $F_{012}^{*}$ \\
\hline \multicolumn{13}{|c|}{ Single volatility shift, $\delta=3, \tau=0.2$} \\
\hline \multirow[t]{8}{*}{$\operatorname{AR}(4)$} & -0.8 & 50 & 13.6 & 24.4 & 37.4 & 48.3 & 52.1 & 8.8 & 11.9 & 13.0 & 12.9 & 11.8 \\
\hline & & 100 & 18.6 & 26.7 & 41.8 & 53.0 & 57.9 & 7.8 & 8.4 & 9.4 & 9.3 & 8.0 \\
\hline & -0.5 & 50 & 15.8 & 25.2 & 39.1 & 50.6 & 55.0 & 10.0 & 12.6 & 13.4 & 13.2 & 12.3 \\
\hline & & 100 & 20.7 & 27.7 & 41.9 & 53.2 & 59.7 & 7.9 & 8.6 & 9.0 & 8.7 & 8.1 \\
\hline & 0.5 & 50 & 13.3 & 23.5 & 35.6 & 45.3 & 47.3 & 8.0 & 10.5 & 10.6 & 9.6 & 8.5 \\
\hline & & 100 & 17.8 & 26.8 & 40.4 & 51.6 & 55.8 & 7.4 & 8.2 & 7.7 & 7.2 & 6.4 \\
\hline & 0.8 & 50 & 12.4 & 20.1 & 27.9 & 35.3 & 36.2 & 7.2 & 9.2 & 6.7 & 6.0 & 5.3 \\
\hline & & 100 & 15.5 & 24.3 & 35.0 & 44.2 & 47.5 & 6.4 & 6.7 & 5.7 & 4.9 & 4.3 \\
\hline \multirow[t]{4}{*}{$\operatorname{MA}(2)$} & -0.5 & 50 & 22.7 & 31.1 & 35.2 & 50.7 & 59.5 & 14.6 & 14.3 & 11.9 & 13.1 & 14.2 \\
\hline & & 100 & 25.0 & 31.6 & 40.0 & 54.0 & 62.6 & 10.5 & 9.1 & 9.1 & 9.7 & 9.6 \\
\hline & 0.5 & 50 & 14.1 & 24.0 & 45.4 & 54.9 & 57.2 & 8.7 & 11.1 & 13.9 & 13.2 & 11.9 \\
\hline & & 100 & 17.7 & 26.5 & 45.9 & 55.8 & 59.3 & 6.9 & 8.4 & 8.7 & 8.4 & 7.5 \\
\hline \multicolumn{13}{|c|}{ Single periodic volatility shift, $\delta=3, \tau=0.2$, Case 3} \\
\hline \multirow[t]{8}{*}{$\operatorname{AR}(4)$} & -0.8 & 50 & 3.2 & 6.4 & 8.4 & 9.2 & 9.2 & 4.7 & 6.6 & 7.1 & 6.2 & 5.4 \\
\hline & & 100 & 5.0 & 7.3 & 9.6 & 10.7 & 11.3 & 4.9 & 5.8 & 5.8 & 5.6 & 5.0 \\
\hline & -0.5 & 50 & 4.1 & 6.9 & 9.5 & 10.1 & 10.6 & 5.1 & 6.6 & 6.6 & 6.1 & 5.5 \\
\hline & & 100 & 5.5 & 7.7 & 10.8 & 11.6 & 11.8 & 5.2 & 6.1 & 5.8 & 5.3 & 4.8 \\
\hline & 0.5 & 50 & 3.7 & 6.2 & 8.7 & 9.0 & 8.8 & 4.7 & 6.0 & 6.0 & 5.2 & 4.7 \\
\hline & & 100 & 5.5 & 7.4 & 10.1 & 11.4 & 11.2 & 5.3 & 5.9 & 5.5 & 5.2 & 4.8 \\
\hline & 0.8 & 50 & 5.0 & 6.8 & 7.9 & 8.7 & 9.1 & 5.7 & 6.3 & 5.4 & 4.9 & 4.5 \\
\hline & & 100 & 5.2 & 7.4 & 9.2 & 10.0 & 10.0 & 5.0 & 6.0 & 5.0 & 4.9 & 4.8 \\
\hline \multirow[t]{4}{*}{$\mathrm{MA}(2)$} & -0.5 & 50 & 9.5 & 11.2 & 8.7 & 12.2 & 16.8 & 11.0 & 10.3 & 7.0 & 8.0 & 10.4 \\
\hline & & 100 & 9.3 & 10.1 & 9.7 & 12.6 & 15.7 & 8.8 & 7.7 & 5.9 & 6.5 & 7.6 \\
\hline & 0.5 & 50 & 4.1 & 5.9 & 14.4 & 14.5 & 13.6 & 5.2 & 6.5 & 10.4 & 8.7 & 7.6 \\
\hline & & 100 & 4.4 & 6.8 & 14.1 & 14.1 & 13.0 & 4.6 & 5.4 & 8.0 & 6.9 & 5.9 \\
\hline
\end{tabular}


Table S.4. Empirical size of conventional and wild bootstrap HEGY tests using Step 2b of Algorithm 1. Local GLS de-trending. Weakly dependent shocks.

\begin{tabular}{|c|c|c|c|c|c|c|c|c|c|c|c|c|}
\hline & $\Phi / \Theta$ & $N$ & $t_{0}$ & $t_{2}$ & $F_{1}$ & $F_{12}$ & $F_{012}$ & $t_{0}^{*}$ & $t_{2}^{*}$ & $F_{1}^{*}$ & $F_{12}^{*}$ & $F_{012}^{*}$ \\
\hline \multicolumn{13}{|c|}{ Homoskedasticity } \\
\hline \multirow{8}{*}{$\operatorname{AR}(4)$} & -0.8 & 50 & 3.5 & 7.4 & 6.2 & 6.6 & 6.2 & 3.5 & 4.3 & 4.8 & 4.6 & 3.9 \\
\hline & & 100 & 4.3 & 6.6 & 5.6 & 5.3 & 5.6 & 4.3 & 4.7 & 4.8 & 4.6 & 4.3 \\
\hline & -0.5 & 50 & 4.4 & 8.2 & 5.7 & 6.7 & 7.0 & 3.7 & 4.6 & 5.0 & 4.4 & 4.4 \\
\hline & & 100 & 4.9 & 6.5 & 5.3 & 5.1 & 5.4 & 4.8 & 5.0 & 4.9 & 5.0 & 4.7 \\
\hline & 0.5 & 50 & 3.8 & 6.8 & 9.0 & 9.1 & 7.6 & 2.6 & 3.9 & 7.3 & 6.2 & 4.2 \\
\hline & & 100 & 5.2 & 6.9 & 5.8 & 5.8 & 6.3 & 5.2 & 4.7 & 4.9 & 4.5 & 4.9 \\
\hline & 0.8 & 50 & 6.5 & 8.7 & 6.9 & 8.1 & 9.6 & 5.5 & 5.2 & 5.9 & 5.7 & 6.0 \\
\hline & & 100 & 5.4 & 7.0 & 5.7 & 6.1 & 6.6 & 4.9 & 5.1 & 5.6 & 5.3 & 5.3 \\
\hline \multirow[t]{4}{*}{$\operatorname{MA}(2)$} & -0.5 & 50 & 12.1 & 15.0 & 6.6 & 11.0 & 16.3 & 10.1 & 10.1 & 5.3 & 7.9 & 11.5 \\
\hline & & 100 & 9.5 & 10.2 & 5.2 & 7.1 & 10.1 & 8.3 & 7.9 & 4.7 & 6.3 & 8.4 \\
\hline & 0.5 & 50 & 5.1 & 8.2 & 8.3 & 8.8 & 9.2 & 4.2 & 4.8 & 6.8 & 6.7 & 5.9 \\
\hline & & 100 & 4.0 & 5.9 & 6.3 & 6.0 & 5.5 & 3.9 & 4.5 & 5.8 & 5.4 & 4.6 \\
\hline \multicolumn{13}{|c|}{ Periodic heteroskedasticity, Case 2} \\
\hline \multirow[t]{8}{*}{$\operatorname{AR}(4)$} & -0.8 & 50 & 5.1 & 8.8 & 9.9 & 13.4 & 12.9 & 4.5 & 5.4 & 5.4 & 5.5 & 5.3 \\
\hline & & 100 & 4.8 & 7.3 & 9.5 & 12.3 & 12.1 & 4.4 & 5.3 & 5.0 & 5.1 & 5.2 \\
\hline & -0.5 & 50 & 5.8 & 8.8 & 9.9 & 13.2 & 13.4 & 5.2 & 5.4 & 5.4 & 5.4 & 5.4 \\
\hline & & 100 & 5.5 & 6.9 & 9.3 & 12.1 & 11.9 & 5.1 & 5.2 & 5.1 & 5.2 & 5.2 \\
\hline & 0.5 & 50 & 4.9 & 7.9 & 10.7 & 13.4 & 13.0 & 4.0 & 4.2 & 5.9 & 5.4 & 5.1 \\
\hline & & 100 & 5.7 & 7.2 & 9.9 & 12.7 & 12.3 & 5.2 & 4.9 & 4.9 & 5.0 & 5.0 \\
\hline & 0.8 & 50 & 6.9 & 9.2 & 10.0 & 13.3 & 13.7 & 6.0 & 5.7 & 5.9 & 5.8 & 6.0 \\
\hline & & 100 & 5.9 & 7.2 & 9.6 & 12.2 & 12.3 & 5.3 & 5.0 & 5.2 & 5.1 & 5.2 \\
\hline \multirow[t]{4}{*}{$\mathrm{MA}(2)$} & -0.5 & 50 & 9.5 & 11.4 & 9.6 & 14.0 & 16.2 & 8.7 & 7.6 & 5.3 & 5.9 & 6.7 \\
\hline & & 100 & 7.1 & 8.2 & 8.8 & 12.4 & 13.0 & 6.9 & 6.1 & 4.9 & 5.1 & 5.7 \\
\hline & 0.5 & 50 & 5.2 & 8.4 & 12.3 & 15.3 & 14.9 & 4.8 & 5.2 & 7.5 & 6.7 & 6.5 \\
\hline & & 100 & 4.9 & 6.4 & 10.3 & 12.7 & 12.2 & 4.5 & 4.8 & 6.0 & 5.5 & 5.5 \\
\hline \multicolumn{13}{|c|}{ Single volatility shift, $\delta=3, \tau=0.8$} \\
\hline \multirow[t]{8}{*}{$\operatorname{AR}(4)$} & -0.8 & 50 & 4.5 & 8.2 & 4.8 & 5.9 & 6.7 & 4.0 & 4.3 & 5.3 & 5.1 & 4.4 \\
\hline & & 100 & 5.0 & 7.1 & 4.6 & 4.9 & 5.6 & 4.1 & 5.0 & 4.8 & 4.7 & 4.3 \\
\hline & -0.5 & 50 & 5.1 & 8.7 & 4.9 & 6.2 & 7.3 & 3.8 & 4.8 & 5.4 & 5.1 & 4.6 \\
\hline & & 100 & 5.6 & 7.3 & 4.4 & 5.0 & 5.8 & 4.4 & 4.9 & 5.0 & 5.0 & 4.9 \\
\hline & 0.5 & 50 & 4.9 & 7.9 & 5.3 & 6.1 & 6.4 & 3.6 & 3.9 & 5.4 & 4.5 & 4.0 \\
\hline & & 100 & 5.8 & 7.2 & 4.2 & 4.8 & 5.8 & 4.9 & 5.1 & 4.6 & 4.7 & 4.8 \\
\hline & 0.8 & 50 & 6.7 & 8.5 & 4.3 & 5.6 & 7.4 & 4.9 & 4.6 & 4.4 & 4.4 & 4.7 \\
\hline & & 100 & 5.8 & 7.0 & 3.5 & 4.5 & 5.6 & 5.0 & 4.7 & 4.3 & 4.3 & 4.7 \\
\hline \multirow[t]{4}{*}{$\mathrm{MA}(2)$} & -0.5 & 50 & 13.3 & 15.4 & 4.9 & 9.6 & 16.5 & 10.9 & 10.0 & 4.7 & 7.7 & 11.5 \\
\hline & & 100 & 9.6 & 11.0 & 4.2 & 6.6 & 10.3 & 8.9 & 7.3 & 4.6 & 6.1 & 8.3 \\
\hline & 0.5 & 50 & 5.8 & 8.5 & 7.3 & 8.4 & 9.6 & 4.5 & 4.9 & 8.0 & 7.1 & 6.1 \\
\hline & & 100 & 4.6 & 6.5 & 5.6 & 5.2 & 5.6 & 3.7 & 4.5 & 6.1 & 5.7 & 4.7 \\
\hline
\end{tabular}


Table S.4. Continued.

\begin{tabular}{|c|c|c|c|c|c|c|c|c|c|c|c|c|}
\hline & $\Phi / \Theta$ & $N$ & $t_{0}$ & $t_{2}$ & $F_{1}$ & $F_{12}$ & $F_{012}$ & $t_{0}^{*}$ & $t_{2}^{*}$ & $F_{1}^{*}$ & $F_{12}^{*}$ & $F_{012}^{*}$ \\
\hline \multicolumn{13}{|c|}{ Single volatility shift, $\delta=3, \tau=0.2$} \\
\hline \multirow[t]{8}{*}{$\operatorname{AR}(4)$} & -0.8 & 50 & 9.0 & 12.6 & 6.7 & 9.0 & 11.8 & 6.8 & 7.9 & 7.6 & 8.9 & 8.7 \\
\hline & & 100 & 8.5 & 10.3 & 5.3 & 7.4 & 8.8 & 5.9 & 6.1 & 6.8 & 6.9 & 6.4 \\
\hline & -0.5 & 50 & 10.5 & 13.2 & 6.6 & 9.7 & 13.1 & 7.2 & 7.6 & 7.6 & 8.2 & 8.7 \\
\hline & & 100 & 9.6 & 9.8 & 5.2 & 6.7 & 9.3 & 5.9 & 6.3 & 6.5 & 6.7 & 6.7 \\
\hline & 0.5 & 50 & 8.6 & 10.2 & 5.0 & 6.7 & 8.8 & 5.6 & 5.5 & 5.2 & 5.5 & 5.3 \\
\hline & & 100 & 8.7 & 9.1 & 4.4 & 5.9 & 8.2 & 5.8 & 5.6 & 5.5 & 5.4 & 5.8 \\
\hline & 0.8 & 50 & 8.0 & 8.0 & 3.8 & 5.1 & 7.2 & 5.5 & 4.1 & 4.1 & 3.8 & 4.6 \\
\hline & & 100 & 7.2 & 7.7 & 3.1 & 4.2 & 6.0 & 4.4 & 4.4 & 3.9 & 3.9 & 4.1 \\
\hline \multirow[t]{4}{*}{$\operatorname{MA}(2)$} & -0.5 & 50 & 19.4 & 20.7 & 5.6 & 13.2 & 22.1 & 14.6 & 13.6 & 6.2 & 11.0 & 16.3 \\
\hline & & 100 & 14.6 & 14.9 & 4.5 & 8.9 & 14.7 & 11.0 & 9.8 & 6.0 & 8.7 & 11.6 \\
\hline & 0.5 & 50 & 9.6 & 11.7 & 10.2 & 12.6 & 15.2 & 6.7 & 6.9 & 10.8 & 10.4 & 9.4 \\
\hline & & 100 & 7.6 & 8.5 & 7.1 & 7.5 & 8.7 & 5.2 & 5.4 & 8.3 & 7.9 & 6.9 \\
\hline \multicolumn{13}{|c|}{ Single periodic volatility shift, $\delta=3, \tau=0.2$, Case 3} \\
\hline \multirow[t]{8}{*}{$\operatorname{AR}(4)$} & -0.8 & 50 & 4.0 & 8.0 & 4.7 & 5.8 & 6.1 & 3.8 & 5.2 & 5.1 & 5.3 & 4.5 \\
\hline & & 100 & 4.6 & 6.3 & 4.6 & 4.6 & 5.5 & 4.2 & 5.0 & 4.9 & 4.9 & 4.3 \\
\hline & -0.5 & 50 & 5.0 & 8.6 & 4.5 & 5.9 & 7.0 & 4.4 & 5.1 & 5.6 & 4.9 & 5.0 \\
\hline & & 100 & 5.8 & 6.5 & 4.3 & 4.8 & 5.8 & 4.8 & 5.2 & 5.7 & 5.6 & 5.2 \\
\hline & 0.5 & 50 & 4.5 & 7.3 & 6.0 & 6.5 & 7.0 & 3.8 & 4.1 & 6.1 & 5.6 & 4.5 \\
\hline & & 100 & 5.4 & 6.9 & 4.2 & 4.8 & 5.8 & 5.3 & 4.8 & 5.1 & 5.0 & 5.2 \\
\hline & 0.8 & 50 & 6.6 & 8.6 & 4.3 & 5.7 & 8.3 & 5.4 & 5.3 & 5.2 & 5.2 & 5.6 \\
\hline & & 100 & 5.2 & 6.3 & 4.4 & 4.9 & 5.8 & 4.8 & 5.0 & 5.0 & 5.3 & 5.0 \\
\hline \multirow[t]{4}{*}{$\mathrm{MA}(2)$} & -0.5 & 50 & 13.0 & 15.8 & 4.4 & 9.4 & 16.4 & 11.7 & 10.8 & 5.0 & 8.7 & 12.2 \\
\hline & & 100 & 9.8 & 10.5 & 4.0 & 6.2 & 10.3 & 8.9 & 8.0 & 4.9 & 6.6 & 8.4 \\
\hline & 0.5 & 50 & 5.4 & 7.9 & 8.0 & 8.7 & 9.7 & 4.9 & 5.2 & 9.0 & 7.6 & 7.0 \\
\hline & & 100 & 4.2 & 6.2 & 6.1 & 5.8 & 5.8 & 4.0 & 4.8 & 7.4 & 6.8 & 5.6 \\
\hline
\end{tabular}


Table S.5. Finite sample size-adjusted power of conventional and wild bootstrap HEGY tests using Step 2b of Algorithm 1. OLS de-trending.

\begin{tabular}{|c|c|c|c|c|c|c|c|c|c|c|c|c|c|c|}
\hline$\Sigma$ & $\delta$ & $\tau$ & $-c$ & $N$ & $t_{0}$ & $t_{2}$ & $F_{1}$ & $F_{12}$ & $F_{012}$ & $t_{0}^{*}$ & $t_{2}^{*}$ & $F_{1}^{*}$ & $F_{12}^{*}$ & $F_{012}^{*}$ \\
\hline \multicolumn{15}{|c|}{ Homoskedasticity } \\
\hline & & & \multirow[t]{2}{*}{3.75} & 50 & 6.9 & 14.3 & 19.0 & 24.5 & 22.3 & 6.6 & 13.4 & 18.5 & 24.6 & 21.9 \\
\hline & & & & 100 & 7.3 & 14.4 & 20.7 & 27.2 & 25.2 & 6.9 & 14.3 & 19.9 & 27.3 & 23.9 \\
\hline & & & \multirow[t]{2}{*}{7} & 50 & 13.7 & 31.1 & 44.5 & 58.8 & 57.4 & 13.2 & 29.1 & 44.7 & 58.6 & 56.4 \\
\hline & & & & 100 & 13.7 & 29.5 & 46.6 & 61.5 & 61.2 & 12.7 & 29.4 & 46.1 & 61.5 & 58.8 \\
\hline & & & \multirow[t]{2}{*}{13.5} & 50 & 41.9 & 72.5 & 89.2 & 95.4 & 95.6 & 37.4 & 69.1 & 89.9 & 96.1 & 96.2 \\
\hline & & & & 100 & 38.5 & 69.3 & 91.3 & 97.6 & 98.1 & 36.0 & 68.3 & 91.4 & 97.7 & 97.9 \\
\hline \multicolumn{15}{|c|}{ Periodic heteroskedasticity } \\
\hline \multirow[t]{6}{*}{ Case 1} & & & 3.75 & 50 & 8.6 & 14.9 & 20.7 & 25.3 & 22.1 & 7.7 & 14.5 & 20.1 & 24.9 & 22.1 \\
\hline & & & & 100 & 8.9 & 15.0 & 21.3 & 27.3 & 24.7 & 8.3 & 14.9 & 20.9 & 27.0 & 23.7 \\
\hline & & & 7 & 50 & 16.1 & 31.0 & 43.3 & 55.1 & 52.0 & 13.8 & 29.5 & 42.3 & 55.3 & 51.0 \\
\hline & & & & 100 & 15.3 & 30.1 & 44.4 & 58.0 & 54.6 & 13.7 & 29.9 & 43.5 & 57.7 & 53.3 \\
\hline & & & 13.5 & 50 & 45.6 & 71.1 & 84.1 & 93.4 & 92.6 & 38.9 & 68.4 & 83.4 & 93.8 & 93.1 \\
\hline & & & & 100 & 40.5 & 69.2 & 85.2 & 95.2 & 94.9 & 36.8 & 67.6 & 84.1 & 95.3 & 94.5 \\
\hline \multirow[t]{6}{*}{ Case 2} & & & 3.75 & 50 & 12.5 & 16.1 & 15.7 & 15.8 & 16.2 & 10.8 & 15.8 & 15.5 & 15.6 & 15.8 \\
\hline & & & & 100 & 11.2 & 15.5 & 15.3 & 15.2 & 15.7 & 9.7 & 15.4 & 15.4 & 15.5 & 15.8 \\
\hline & & & 7 & 50 & 21.9 & 31.9 & 31.5 & 31.9 & 31.6 & 17.8 & 31.5 & 31.7 & 31.3 & 31.1 \\
\hline & & & & 100 & 19.7 & 31.4 & 31.5 & 31.1 & 31.1 & 16.6 & 31.1 & 31.8 & 31.5 & 30.7 \\
\hline & & & 13.5 & 50 & 53.2 & 70.2 & 69.6 & 69.8 & 69.3 & 44.7 & 69.6 & 69.8 & 69.5 & 68.3 \\
\hline & & & & 100 & 47.5 & 69.3 & 69.7 & 69.2 & 68.9 & 40.2 & 69.5 & 69.9 & 69.7 & 67.9 \\
\hline \multicolumn{15}{|c|}{ Single volatility shift } \\
\hline \multirow[t]{24}{*}{ Case 1} & 0.33 & 0.2 & 3.75 & 50 & 6.9 & 14.3 & 19.0 & 24.5 & 22.3 & 6.6 & 13.4 & 18.5 & 24.6 & 21.9 \\
\hline & & & & 100 & 7.3 & 14.4 & 20.7 & 27.2 & 25.2 & 6.9 & 14.3 & 19.9 & 27.3 & 23.9 \\
\hline & & & 7 & 50 & 13.7 & 31.1 & 44.5 & 58.8 & 57.4 & 13.2 & 29.1 & 44.7 & 58.6 & 56.4 \\
\hline & & & & 100 & 13.7 & 29.5 & 46.6 & 61.5 & 61.2 & 12.7 & 29.4 & 46.1 & 61.5 & 58.8 \\
\hline & & & 13.5 & 50 & 41.9 & 72.5 & 89.2 & 95.4 & 95.6 & 37.4 & 69.1 & 89.9 & 96.1 & 96.2 \\
\hline & & & & 100 & 38.5 & 69.3 & 91.3 & 97.6 & 98.1 & 36.0 & 68.3 & 91.4 & 97.7 & 97.9 \\
\hline & & 0.8 & 3.75 & 50 & 8.4 & 11.9 & 12.9 & 15.4 & 16.3 & 8.4 & 11.6 & 13.4 & 15.2 & 15.3 \\
\hline & & & & 100 & 8.8 & 11.8 & 13.4 & 15.6 & 16.3 & 8.5 & 11.7 & 13.3 & 15.1 & 15.3 \\
\hline & & & 7 & 50 & 15.1 & 22.1 & 26.2 & 34.1 & 37.7 & 14.6 & 21.3 & 26.4 & 32.6 & 35.6 \\
\hline & & & & 100 & 14.9 & 21.0 & 26.7 & 33.6 & 37.7 & 14.2 & 20.8 & 26.0 & 33.0 & 36.0 \\
\hline & & & 13.5 & 50 & 35.7 & 48.7 & 60.9 & 73.6 & 78.4 & 33.3 & 47.3 & 61.4 & 73.5 & 78.3 \\
\hline & & & & 100 & 33.8 & 46.9 & 61.7 & 75.8 & 81.8 & 31.7 & 45.9 & 61.1 & 75.2 & 81.3 \\
\hline & 3 & 0.2 & 3.75 & 50 & 10.1 & 3.5 & 2.4 & 1.5 & 1.8 & 9.2 & 3.5 & 2.1 & 1.2 & 1.5 \\
\hline & & & & 100 & 7.2 & 2.1 & 1.7 & 0.7 & 1.1 & 7.0 & 2.2 & 1.5 & 0.9 & 1.1 \\
\hline & & & 7 & 50 & 11.3 & 6.5 & 4.9 & 4.1 & 4.5 & 10.0 & 5.7 & 4.3 & 3.3 & 3.3 \\
\hline & & & & 100 & 7.5 & 3.8 & 3.2 & 2.3 & 2.7 & 7.2 & 3.8 & 3.2 & 2.3 & 2.4 \\
\hline & & & 13.5 & 50 & 24.7 & 22.2 & 27.2 & 31.5 & 34.2 & 20.5 & 18.4 & 21.1 & 21.5 & 22.8 \\
\hline & & & & 100 & 16.5 & 14.4 & 18.1 & 20.2 & 24.8 & 15.2 & 12.9 & 14.8 & 16.9 & 19.2 \\
\hline & & 0.8 & 3.75 & 50 & 6.5 & 8.4 & 9.9 & 12.0 & 11.7 & 6.1 & 8.3 & 9.7 & 11.1 & 9.9 \\
\hline & & & & 100 & 5.8 & 8.4 & 10.5 & 11.8 & 11.1 & 5.8 & 7.9 & 9.7 & 11.4 & 10.8 \\
\hline & & & 7 & 50 & 11.0 & 16.8 & 24.2 & 33.2 & 33.8 & 9.8 & 15.6 & 23.2 & 29.7 & 29.2 \\
\hline & & & & 100 & 9.6 & 15.6 & 24.5 & 32.0 & 32.7 & 9.2 & 14.8 & 22.9 & 31.0 & 30.4 \\
\hline & & & 13.5 & 50 & 34.0 & 52.5 & 77.2 & 89.4 & 90.5 & 29.0 & 46.6 & 71.9 & 84.6 & 86.0 \\
\hline & & & & 100 & 28.6 & 47.5 & 76.3 & 89.3 & 91.2 & 25.2 & 43.6 & 72.1 & 86.7 & 88.9 \\
\hline
\end{tabular}

$[\mathrm{S} .15]$ 
Table S.5. Continued.

\begin{tabular}{|c|c|c|c|c|c|c|c|c|c|c|c|c|c|c|}
\hline$\Sigma$ & $\delta$ & $\tau$ & $-c$ & $N$ & $t_{0}$ & $t_{2}$ & $F_{1}$ & $F_{12}$ & $F_{012}$ & $t_{0}^{*}$ & $t_{2}^{*}$ & $F_{1}^{*}$ & $F_{12}^{*}$ & $F_{012}^{*}$ \\
\hline \multicolumn{15}{|c|}{ Single periodic volatility shift } \\
\hline \multirow[t]{24}{*}{ Case 3} & 0.33 & 0.2 & 3.75 & 50 & 6.9 & 14.3 & 19.0 & 24.5 & 22.3 & 6.6 & 13.4 & 18.5 & 24.6 & 21.9 \\
\hline & & & & 100 & 7.3 & 14.4 & 20.7 & 27.2 & 25.2 & 6.9 & 14.3 & 19.9 & 27.3 & 23.9 \\
\hline & & & 7 & 50 & 13.7 & 31.1 & 44.5 & 58.8 & 57.4 & 13.2 & 29.1 & 44.7 & 58.6 & 56.4 \\
\hline & & & & 100 & 13.7 & 29.5 & 46.6 & 61.5 & 61.2 & 12.7 & 29.4 & 46.1 & 61.5 & 58.8 \\
\hline & & & 13.5 & 50 & 41.9 & 72.5 & 89.2 & 95.4 & 95.6 & 37.4 & 69.1 & 89.9 & 96.1 & 96.2 \\
\hline & & & & 100 & 38.5 & 69.3 & 91.3 & 97.6 & 98.1 & 36.0 & 68.3 & 91.4 & 97.7 & 97.9 \\
\hline & & 0.8 & 3.75 & 50 & 8.4 & 11.9 & 12.9 & 15.4 & 16.3 & 8.4 & 11.6 & 13.4 & 15.2 & 15.3 \\
\hline & & & & 100 & 8.8 & 11.8 & 13.4 & 15.6 & 16.3 & 8.5 & 11.7 & 13.3 & 15.1 & 15.3 \\
\hline & & & 7 & 50 & 15.1 & 22.1 & 26.2 & 34.1 & 37.7 & 14.6 & 21.3 & 26.4 & 32.6 & 35.6 \\
\hline & & & & 100 & 14.9 & 21.0 & 26.7 & 33.6 & 37.7 & 14.2 & 20.8 & 26.0 & 33.0 & 36.0 \\
\hline & & & 13.5 & 50 & 35.7 & 48.7 & 60.9 & 73.6 & 78.4 & 33.3 & 47.3 & 61.4 & 73.5 & 78.3 \\
\hline & & & & 100 & 33.8 & 46.9 & 61.7 & 75.8 & 81.8 & 31.7 & 45.9 & 61.1 & 75.2 & 81.3 \\
\hline & 3 & 0.2 & 3.75 & 50 & 10.1 & 3.5 & 2.4 & 1.5 & 1.8 & 9.2 & 3.5 & 2.1 & 1.2 & 1.5 \\
\hline & & & & 100 & 7.2 & 2.1 & 1.7 & 0.7 & 1.1 & 7.0 & 2.2 & 1.5 & 0.9 & 1.1 \\
\hline & & & 7 & 50 & 11.3 & 6.5 & 4.9 & 4.1 & 4.5 & 10.0 & 5.7 & 4.3 & 3.3 & 3.3 \\
\hline & & & & 100 & 7.5 & 3.8 & 3.2 & 2.3 & 2.7 & 7.2 & 3.8 & 3.2 & 2.3 & 2.4 \\
\hline & & & 13.5 & 50 & 24.7 & 22.2 & 27.2 & 31.5 & 34.2 & 20.5 & 18.4 & 21.1 & 21.5 & 22.8 \\
\hline & & & & 100 & 16.5 & 14.4 & 18.1 & 20.2 & 24.8 & 15.2 & 12.9 & 14.8 & 16.9 & 19.2 \\
\hline & & 0.8 & 3.75 & 50 & 6.5 & 8.4 & 9.9 & 12.0 & 11.7 & 6.1 & 8.3 & 9.7 & 11.1 & 9.9 \\
\hline & & & & 100 & 5.8 & 8.4 & 10.5 & 11.8 & 11.1 & 5.8 & 7.9 & 9.7 & 11.4 & 10.8 \\
\hline & & & 7 & 50 & 11.0 & 16.8 & 24.2 & 33.2 & 33.8 & 9.8 & 15.6 & 23.2 & 29.7 & 29.2 \\
\hline & & & & 100 & 9.6 & 15.6 & 24.5 & 32.0 & 32.7 & 9.2 & 14.8 & 22.9 & 31.0 & 30.4 \\
\hline & & & 13.5 & 50 & 34.0 & 52.5 & 77.2 & 89.4 & 90.5 & 29.0 & 46.6 & 71.9 & 84.6 & 86.0 \\
\hline & & & & 100 & 28.6 & 47.5 & 76.3 & 89.3 & 91.2 & 25.2 & 43.6 & 72.1 & 86.7 & 88.9 \\
\hline \multirow[t]{12}{*}{ Case 4} & & 0.2 & 3.75 & 50 & 10.8 & 4.0 & 2.8 & 1.7 & 2.5 & 10.2 & 3.6 & 2.8 & 1.5 & 2.0 \\
\hline & & & & 100 & 8.6 & 2.2 & 1.4 & 0.9 & 1.3 & 8.1 & 2.4 & 1.8 & 1.0 & 1.3 \\
\hline & & & 7 & 50 & 12.5 & 7.2 & 6.1 & 4.9 & 6.1 & 11.2 & 6.2 & 5.7 & 4.0 & 4.4 \\
\hline & & & & 100 & 8.9 & 3.9 & 3.0 & 2.6 & 3.1 & 8.3 & 4.0 & 3.7 & 2.4 & 3.0 \\
\hline & & & 13.5 & 50 & 26.2 & 23.6 & 26.4 & 29.6 & 33.1 & 22.0 & 19.0 & 21.4 & 22.2 & 23.2 \\
\hline & & & & 100 & 18.5 & 14.4 & 15.7 & 20.0 & 22.4 & 15.9 & 12.9 & 14.7 & 16.5 & 18.2 \\
\hline & & 0.8 & 3.75 & 50 & 6.9 & 9.1 & 12.2 & 12.7 & 12.9 & 6.6 & 8.5 & 10.7 & 11.5 & 10.5 \\
\hline & & & & 100 & 7.2 & 8.7 & 10.9 & 12.2 & 12.2 & 6.5 & 8.2 & 10.7 & 11.9 & 11.5 \\
\hline & & & 7 & 50 & 11.6 & 17.7 & 26.2 & 32.6 & 33.1 & 10.4 & 16.1 & 22.4 & 28.0 & 27.2 \\
\hline & & & & 100 & 11.0 & 16.7 & 22.7 & 30.7 & 30.5 & 10.2 & 15.1 & 21.1 & 28.8 & 27.5 \\
\hline & & & 13.5 & 50 & 35.2 & 52.7 & 71.1 & 84.6 & 85.2 & 29.9 & 46.8 & 62.3 & 77.3 & 77.4 \\
\hline & & & & 100 & 30.3 & 47.6 & 64.6 & 82.4 & 82.7 & 26.7 & 43.7 & 60.4 & 78.4 & 78.2 \\
\hline
\end{tabular}


Table S.6. Finite sample size-adjusted power of conventional and wild bootstrap HEGY tests using Step 2b of Algorithm 1. Local GLS de-trending.

\begin{tabular}{|c|c|c|c|c|c|c|c|c|c|c|c|c|c|c|}
\hline$\Sigma$ & $\delta$ & $\tau$ & $-c$ & $N$ & $t_{0}$ & $t_{2}$ & $F_{1}$ & $F_{12}$ & $F_{012}$ & $t_{0}^{*}$ & $t_{2}^{*}$ & $F_{1}^{*}$ & $F_{12}^{*}$ & $F_{012}^{*}$ \\
\hline \multicolumn{15}{|c|}{ Homoskedasticity } \\
\hline & & & \multirow[t]{2}{*}{3.75} & 50 & 8.3 & 19.0 & 24.5 & 33.2 & 30.0 & 8.4 & 17.7 & 24.1 & 32.8 & 29.0 \\
\hline & & & & 100 & 8.8 & 18.8 & 23.9 & 34.1 & 31.0 & 8.7 & 18.6 & 23.8 & 33.0 & 29.8 \\
\hline & & & \multirow[t]{2}{*}{7} & 50 & 16.5 & 41.9 & 60.8 & 76.9 & 73.2 & 16.0 & 38.8 & 59.8 & 76.2 & 71.4 \\
\hline & & & & 100 & 17.0 & 40.4 & 60.0 & 77.7 & 74.8 & 16.4 & 39.4 & 59.5 & 76.6 & 73.6 \\
\hline & & & \multirow[t]{2}{*}{13.5} & 50 & 48.4 & 84.2 & 96.2 & 98.4 & 98.1 & 44.3 & 80.0 & 96.5 & 98.8 & 98.5 \\
\hline & & & & 100 & 47.1 & 84.0 & 97.6 & 99.6 & 99.5 & 44.5 & 81.9 & 97.6 & 99.6 & 99.6 \\
\hline \multicolumn{15}{|c|}{ Periodic heteroskedasticity } \\
\hline \multirow[t]{6}{*}{ Case 1} & & & 3.75 & 50 & 9.9 & 18.1 & 26.8 & 34.5 & 28.8 & 9.2 & 18.3 & 26.6 & 33.4 & 28.7 \\
\hline & & & & 100 & 10.6 & 19.2 & 26.5 & 34.2 & 30.4 & 9.8 & 18.6 & 25.6 & 33.2 & 29.4 \\
\hline & & & 7 & 50 & 19.1 & 39.3 & 58.5 & 73.9 & 66.8 & 16.7 & 38.6 & 57.3 & 72.6 & 65.7 \\
\hline & & & & 100 & 19.2 & 41.8 & 58.2 & 74.3 & 68.7 & 17.5 & 39.9 & 56.3 & 73.0 & 66.7 \\
\hline & & & 13.5 & 50 & 52.5 & 81.7 & 94.0 & 98.1 & 97.0 & 46.1 & 79.4 & 93.7 & 98.3 & 97.3 \\
\hline & & & & 100 & 50.4 & 84.5 & 95.2 & 99.0 & 98.3 & 45.4 & 82.1 & 94.3 & 98.9 & 98.2 \\
\hline \multirow[t]{6}{*}{ Case 2} & & & 3.75 & 50 & 14.6 & 19.0 & 18.6 & 19.5 & 19.9 & 12.7 & 19.2 & 18.9 & 19.3 & 19.6 \\
\hline & & & & 100 & 13.1 & 19.0 & 17.5 & 17.6 & 19.4 & 11.9 & 18.9 & 17.4 & 17.9 & 19.0 \\
\hline & & & 7 & 50 & 26.1 & 40.1 & 40.9 & 42.2 & 41.4 & 21.3 & 39.8 & 41.3 & 41.7 & 40.8 \\
\hline & & & & 100 & 23.3 & 40.9 & 39.1 & 39.1 & 40.8 & 20.6 & 40.5 & 38.6 & 39.4 & 39.8 \\
\hline & & & 13.5 & 50 & 61.0 & 80.5 & 82.8 & 83.1 & 82.1 & 51.8 & 80.1 & 82.6 & 82.4 & 80.8 \\
\hline & & & & 100 & 55.8 & 83.4 & 82.9 & 82.7 & 83.0 & 49.4 & 82.9 & 82.3 & 82.6 & 81.7 \\
\hline \multicolumn{15}{|c|}{ Single volatility shift } \\
\hline \multirow[t]{24}{*}{ Case 1} & 0.33 & 0.2 & 3.75 & 50 & 8.3 & 19.0 & 24.5 & 33.2 & 30.0 & 8.4 & 17.7 & 24.1 & 32.8 & 29.0 \\
\hline & & & & 100 & 8.8 & 18.8 & 23.9 & 34.1 & 31.0 & 8.7 & 18.6 & 23.8 & 33.0 & 29.8 \\
\hline & & & 7 & 50 & 16.5 & 41.9 & 60.8 & 76.9 & 73.2 & 16.0 & 38.8 & 59.8 & 76.2 & 71.4 \\
\hline & & & & 100 & 17.0 & 40.4 & 60.0 & 77.7 & 74.8 & 16.4 & 39.4 & 59.5 & 76.6 & 73.6 \\
\hline & & & 13.5 & 50 & 48.4 & 84.2 & 96.2 & 98.4 & 98.1 & 44.3 & 80.0 & 96.5 & 98.8 & 98.5 \\
\hline & & & & 100 & 47.1 & 84.0 & 97.6 & 99.6 & 99.5 & 44.5 & 81.9 & 97.6 & 99.6 & 99.6 \\
\hline & & 0.8 & 3.75 & 50 & 9.8 & 19.5 & 19.9 & 23.0 & 22.9 & 9.8 & 18.1 & 18.8 & 22.4 & 22.1 \\
\hline & & & & 100 & 10.2 & 18.8 & 16.5 & 20.5 & 20.1 & 9.9 & 19.1 & 16.5 & 19.5 & 19.9 \\
\hline & & & 7 & 50 & 18.7 & 37.0 & 42.8 & 51.6 & 53.4 & 17.9 & 34.2 & 40.6 & 49.9 & 51.3 \\
\hline & & & & 100 & 19.2 & 36.3 & 39.0 & 48.9 & 51.6 & 17.9 & 36.1 & 37.6 & 47.2 & 50.8 \\
\hline & & & 13.5 & 50 & 43.0 & 67.1 & 78.6 & 86.8 & 88.5 & 40.3 & 63.5 & 76.3 & 86.3 & 88.9 \\
\hline & & & & 100 & 43.8 & 67.4 & 77.6 & 88.3 & 92.0 & 41.2 & 66.3 & 75.6 & 87.9 & 91.8 \\
\hline & 3 & 0.2 & 3.75 & 50 & 10.2 & 24.3 & 47.7 & 55.2 & 47.2 & 9.8 & 22.8 & 45.4 & 54.4 & 44.6 \\
\hline & & & & 100 & 9.7 & 23.3 & 47.0 & 56.6 & 44.5 & 8.9 & 22.8 & 45.8 & 55.3 & 43.9 \\
\hline & & & 7 & 50 & 19.6 & 46.1 & 82.6 & 89.4 & 86.2 & 18.5 & 42.7 & 80.5 & 88.6 & 84.4 \\
\hline & & & & 100 & 18.1 & 46.3 & 82.4 & 91.3 & 86.5 & 16.9 & 44.7 & 81.0 & 90.3 & 85.4 \\
\hline & & & 13.5 & 50 & 48.9 & 81.0 & 99.1 & 99.7 & 99.6 & 46.3 & 78.9 & 99.1 & 99.8 & 99.8 \\
\hline & & & & 100 & 47.4 & 82.6 & 99.3 & 99.9 & 99.8 & 43.5 & 81.0 & 99.4 & 99.9 & 99.8 \\
\hline & & 0.8 & 3.75 & 50 & 7.5 & 21.6 & 37.5 & 48.5 & 40.7 & 7.3 & 20.9 & 37.2 & 49.2 & 38.7 \\
\hline & & & & 100 & 7.6 & 22.2 & 39.6 & 51.3 & 41.6 & 7.2 & 22.1 & 38.8 & 51.4 & 40.6 \\
\hline & & & 7 & 50 & 15.9 & 48.0 & 80.7 & 91.6 & 87.2 & 14.9 & 45.5 & 80.6 & 91.5 & 85.4 \\
\hline & & & & 100 & 15.5 & 49.6 & 82.9 & 94.0 & 89.4 & 14.4 & 47.9 & 81.9 & 93.6 & 88.4 \\
\hline & & & 13.5 & 50 & 50.8 & 89.4 & 99.4 & 99.8 & 99.6 & 46.0 & 86.9 & 99.8 & 99.9 & 99.8 \\
\hline & & & & 100 & 49.7 & 91.7 & 99.8 & 100.0 & 100.0 & 45.8 & 89.9 & 99.8 & 100.0 & 100.0 \\
\hline
\end{tabular}


Table S.6. Continued.

\begin{tabular}{|c|c|c|c|c|c|c|c|c|c|c|c|c|c|c|}
\hline$\Sigma$ & $\delta$ & $\tau$ & $-c$ & $N$ & $t_{0}$ & $t_{2}$ & $F_{1}$ & $F_{12}$ & $F_{012}$ & $t_{0}^{*}$ & $t_{2}^{*}$ & $F_{1}^{*}$ & $F_{12}^{*}$ & $F_{012}^{*}$ \\
\hline \multicolumn{15}{|c|}{ Single periodic volatility shift } \\
\hline \multirow[t]{24}{*}{ Case 3} & 0.33 & 0.2 & 3.75 & 50 & 8.3 & 19.0 & 24.5 & 33.2 & 30.0 & 8.4 & 17.7 & 24.1 & 32.8 & 29.0 \\
\hline & & & & 100 & 8.8 & 18.8 & 23.9 & 34.1 & 31.0 & 8.7 & 18.6 & 23.8 & 33.0 & 29.8 \\
\hline & & & 7 & 50 & 16.5 & 41.9 & 60.8 & 76.9 & 73.2 & 16.0 & 38.8 & 59.8 & 76.2 & 71.4 \\
\hline & & & & 100 & 17.0 & 40.4 & 60.0 & 77.7 & 74.8 & 16.4 & 39.4 & 59.5 & 76.6 & 73.6 \\
\hline & & & 13.5 & 50 & 48.4 & 84.2 & 96.2 & 98.4 & 98.1 & 44.3 & 80.0 & 96.5 & 98.8 & 98.5 \\
\hline & & & & 100 & 47.1 & 84.0 & 97.6 & 99.6 & 99.5 & 44.5 & 81.9 & 97.6 & 99.6 & 99.6 \\
\hline & & 0.8 & 3.75 & 50 & 9.8 & 19.5 & 19.9 & 23.0 & 22.9 & 9.8 & 18.1 & 18.8 & 22.4 & 22.1 \\
\hline & & & & 100 & 10.2 & 18.8 & 16.5 & 20.5 & 20.1 & 9.9 & 19.1 & 16.5 & 19.5 & 19.9 \\
\hline & & & 7 & 50 & 18.7 & 37.0 & 42.8 & 51.6 & 53.4 & 17.9 & 34.2 & 40.6 & 49.9 & 51.3 \\
\hline & & & & 100 & 19.2 & 36.3 & 39.0 & 48.9 & 51.6 & 17.9 & 36.1 & 37.6 & 47.2 & 50.8 \\
\hline & & & 13.5 & 50 & 43.0 & 67.1 & 78.6 & 86.8 & 88.5 & 40.3 & 63.5 & 76.3 & 86.3 & 88.9 \\
\hline & & & & 100 & 43.8 & 67.4 & 77.6 & 88.3 & 92.0 & 41.2 & 66.3 & 75.6 & 87.9 & 91.8 \\
\hline & 3 & 0.2 & 3.75 & 50 & 10.2 & 24.3 & 47.7 & 55.2 & 47.2 & 9.8 & 22.8 & 45.4 & 54.4 & 44.6 \\
\hline & & & & 100 & 9.7 & 23.3 & 47.0 & 56.6 & 44.5 & 8.9 & 22.8 & 45.8 & 55.3 & 43.9 \\
\hline & & & 7 & 50 & 19.6 & 46.1 & 82.6 & 89.4 & 86.2 & 18.5 & 42.7 & 80.5 & 88.6 & 84.4 \\
\hline & & & & 100 & 18.1 & 46.3 & 82.4 & 91.3 & 86.5 & 16.9 & 44.7 & 81.0 & 90.3 & 85.4 \\
\hline & & & 13.5 & 50 & 48.9 & 81.0 & 99.1 & 99.7 & 99.6 & 46.3 & 78.9 & 99.1 & 99.8 & 99.8 \\
\hline & & & & 100 & 47.4 & 82.6 & 99.3 & 99.9 & 99.8 & 43.5 & 81.0 & 99.4 & 99.9 & 99.8 \\
\hline & & 0.8 & 3.75 & 50 & 7.5 & 21.6 & 37.5 & 48.5 & 40.7 & 7.3 & 20.9 & 37.2 & 49.2 & 38.7 \\
\hline & & & & 100 & 7.6 & 22.2 & 39.6 & 51.3 & 41.6 & 7.2 & 22.1 & 38.8 & 51.4 & 40.6 \\
\hline & & & 7 & 50 & 15.9 & 48.0 & 80.7 & 91.6 & 87.2 & 14.9 & 45.5 & 80.6 & 91.5 & 85.4 \\
\hline & & & & 100 & 15.5 & 49.6 & 82.9 & 94.0 & 89.4 & 14.4 & 47.9 & 81.9 & 93.6 & 88.4 \\
\hline & & & 13.5 & 50 & 50.8 & 89.4 & 99.4 & 99.8 & 99.6 & 46.0 & 86.9 & 99.8 & 99.9 & 99.8 \\
\hline & & & & 100 & 49.7 & 91.7 & 99.8 & 100.0 & 100.0 & 45.8 & 89.9 & 99.8 & 100.0 & 100.0 \\
\hline \multirow[t]{12}{*}{ Case 4} & & 0.2 & 3.75 & 50 & 11.6 & 23.7 & 41.5 & 50.5 & 41.5 & 11.2 & 22.5 & 40.4 & 49.3 & 40.5 \\
\hline & & & & 100 & 10.1 & 21.9 & 41.2 & 50.5 & 40.4 & 9.4 & 22.3 & 40.9 & 49.9 & 39.4 \\
\hline & & & 7 & 50 & 22.2 & 44.7 & 73.1 & 84.2 & 77.2 & 20.7 & 42.3 & 71.9 & 83.2 & 76.0 \\
\hline & & & & 100 & 19.5 & 44.1 & 72.8 & 85.8 & 77.4 & 18.0 & 43.9 & 72.4 & 85.0 & 76.0 \\
\hline & & & 13.5 & 50 & 53.0 & 79.6 & 96.8 & 99.2 & 98.5 & 48.7 & 77.3 & 96.4 & 99.5 & 98.8 \\
\hline & & & & 100 & 48.9 & 80.9 & 96.8 & 99.6 & 98.8 & 44.5 & 79.9 & 96.4 & 99.5 & 98.8 \\
\hline & & 0.8 & 3.75 & 50 & 7.9 & 22.5 & 38.3 & 47.6 & 37.9 & 7.9 & 21.2 & 36.7 & 47.2 & 37.0 \\
\hline & & & & 100 & 8.7 & 22.9 & 38.3 & 50.3 & 38.3 & 8.1 & 22.3 & 37.9 & 48.7 & 37.5 \\
\hline & & & 7 & 50 & 17.2 & 47.6 & 75.4 & 87.3 & 78.3 & 15.8 & 44.7 & 73.0 & 86.9 & 76.6 \\
\hline & & & & 100 & 18.0 & 49.9 & 75.1 & 90.5 & 80.7 & 15.8 & 47.4 & 74.2 & 89.4 & 79.6 \\
\hline & & & 13.5 & 50 & 52.6 & 89.0 & 98.8 & 99.6 & 99.2 & 47.7 & 86.3 & 98.7 & 99.8 & 99.3 \\
\hline & & & & 100 & 51.9 & 91.5 & 99.2 & 99.9 & 99.7 & 46.7 & 89.6 & 99.1 & 99.9 & 99.7 \\
\hline
\end{tabular}

